\title{
Epigenetic regulation by BAF (mSWI/SNF) chromatin remodeling complexes in late cortical development and beyond
}

\author{
Dissertation \\ for the award of the degree \\ “Doctor of Philosophy” (Ph.D.) \\ of the Georg-August-University of Goettingen \\ within the doctoral program \\ of the Georg-August University School of Science (GAUSS) \\ Submitted by \\ Huong Nguyen \\ from Bac Giang, Vietnam
}

Goettingen, 2019 


\section{Thesis Committee}

Prof. Dr. Jochen Staiger

Department of Neuroanatomy,

University Medical Center Goettingen

Prof. Dr. Gerhard Braus

Department of Molecular Microbiology and Genetics,

University of Goettingen

Prof. Dr. Thomas Dresbach

Department of Anatomy and Embryology,

University of Goettingen

Members of the Examination Board:

Prof. Dr. Jochen Staiger

Department of Neuroanatomy,

University Medical Center Goettingen

Prof. Dr. Gerhard Braus

Department of Molecular Microbiology and Genetics,

University of Goettingen

Prof. Dr. Thomas Dresbach

Department of Anatomy and Embryology,

University of Goettingen

Further members of the Examination Board:

Prof. Gregor Eichele,

Max Planck Institute for Biophysical Chemistry, Goettingen

Prof. Anastassia Stoykova

Max Planck Institute for Biophysical Chemistry, Goettingen

Prof. Dr. André Fiala

Department of Molecular Neurobiology of Behavior

Date of the oral examination: 03.07.2019 


\section{Affidavit}

I herewith declare that the $\mathrm{PhD}$ thesis entitled "Epigenetic regulation by BAF (mSWI/SNF) chromatin remodeling complexes in late cortical development and beyond" was written independently, with no other sources and aids than quoted.

Goettingen, May 22 $2^{\text {th }}, 2019$

Huong Nguyen 


\section{Acknowledgements}

First of all, I would like to thank Prof. Staiger for giving me opportunity to work in his institute and supporting me during my $\mathrm{PhD}$ time.

I would like to thank Dr. Tuoc Tran for giving me the chance to work in his research group. I am very thankful for being always available for discussions, answering questions and for always being positive.

I owe many thanks to the members of my thesis committee, Prof. Staiger, Prof. Braus and Prof. Dresbach for their scientific advice during my PhD period.

I would like to thank members of my Molecular Neurobiology Group: Godwin Sokpor for his collegiality, cooperation and great scientific discussion. Many thanks go especially to our group assistants Linh Pham for her technical helps. Furthermore, I want to extend my thanks to members of the institute for Neuroanatomy lab for their direct or indirect contribution to my project.

I would also like to thank my husband, my son, my parents and the rest of my family for their enormous support during my studies, and for making my life happy! 


\section{Table of Contents}

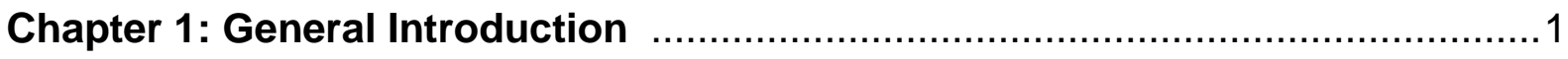

1.1. Epigenetic modifications in cell biological processes....................................

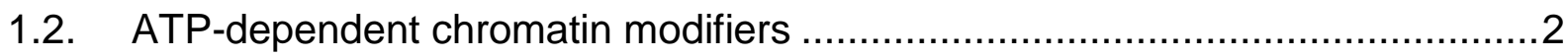

1.3. Biochemical features of the SWI/SNF (BAF) Complex ..................................

1.4. Regulation of cortical development by the mammalian SWI/SNF (BAF)

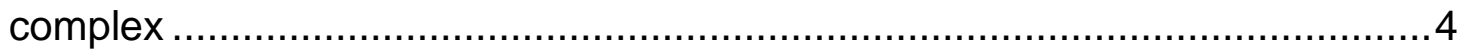

Chapter 2: Epigenetic regulation by BAF (mSWI/SNF) chromatin remodeling complexes is indispensable for embryonic development ..................................

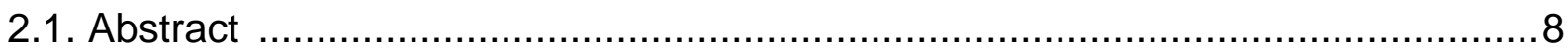

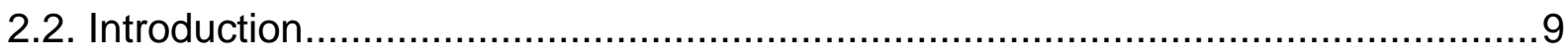

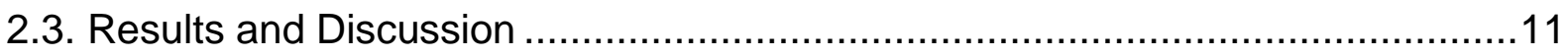

2.3.1. BAF155 and BAF170 are indispensable for brain development and embryogenesis.

2.3.2. BAF155 and BAF170 control the stability of BAF complexes in both cultured cells and embryos.

2.3.3. The loss of BAF complexes induces the accumulation of H3K27me2/3-marked heterochromatin 16

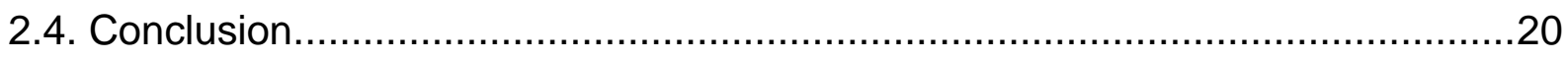

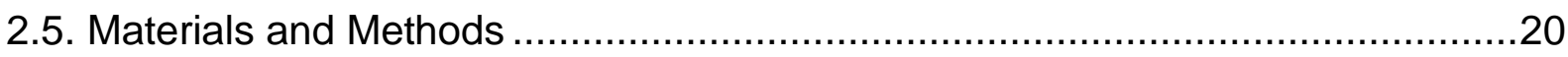

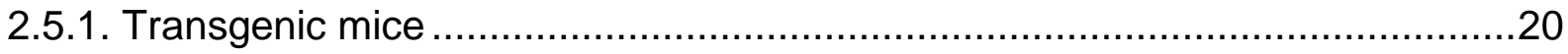

2.5.2. Immunohistochemistry (IHC) and Western blotting (WB) ...........................20

2.5.3. Imaging and quantitative and statistical analyses.....................................21 


\section{Chapter 3: Epigenetic Regulation by BAF Complexes Limits Neural Stem Cell}

Proliferation by Suppressing Wnt Signaling in Late Embryonic Development .22

3.1. Summary .22

3.2. Introduction .23

3.3. Results 25

3.3.1. Loss of BAF complexes causes a genome-wide increase in the level of both active and repressive epigenetic marks at distinct loci in the developing pallium during late neurogenesis.

3.3.2. Conditional inactivation of BAF complexes during late cortical development impairs neurogenesis of upper cortical layer neurons and the hippocampus.

3.3.3. The NSC pool is increased at late development stages in the dcKO pallium ..33

3.3.4. RGs acquire a NE-like identity in the BAF155/BAF170-deficient pallium. .37

3.3.5. Change in spindle orientation, and increased proliferative capacity of NSCs in the BAF155/BAF170-deficient pallium.

3.3.6. Elimination of BAF155 and BAF170 de-represses Wnt signaling in late corticogenesis.

3.4. Discussion

3.4.1. BAF155/BAF170-dependent maintenance of $R G$ cell fate during late cortical neurogenesis.

3.4.2. BAF complexes control NSC proliferation and differentiation in early and late embryonic stages via distinct epigenetic mechanisms.

\subsubsection{BAF complexes suppress Wnt signaling activity $\quad 50$}

3.5. Materials and Methods

3.5.1. Materials

3.5.2. Methods. 


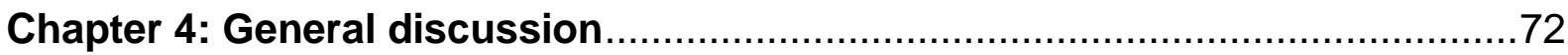

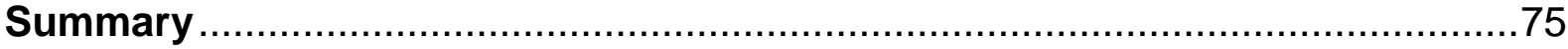

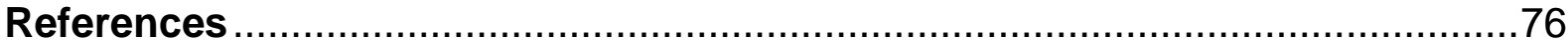

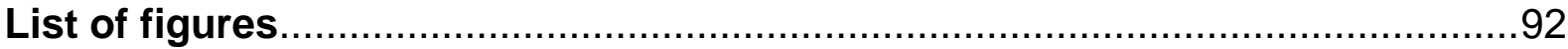

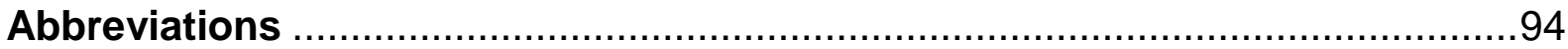

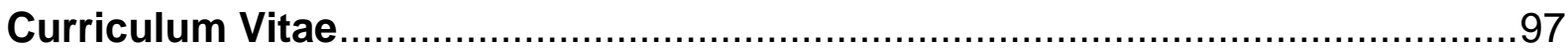




\section{Chapter 1: General Introduction}

\subsection{Epigenetic modifications in cell biological processes}

Epigenetic modifications are defined as mechanisms that regulate gene expression without changes in the underlying DNA sequence (Bernstein et al., 2007; Bird, 2007). In the mammalian cells, epigenetic modifiers can alter chromatin architecture and genomic function through different processes, including DNA, RNA or histone modifications, and activity of non-coding RNAs (Strahl \& Allis, 2000; Goldberg et al., 2007; Kouzarides, 2007).

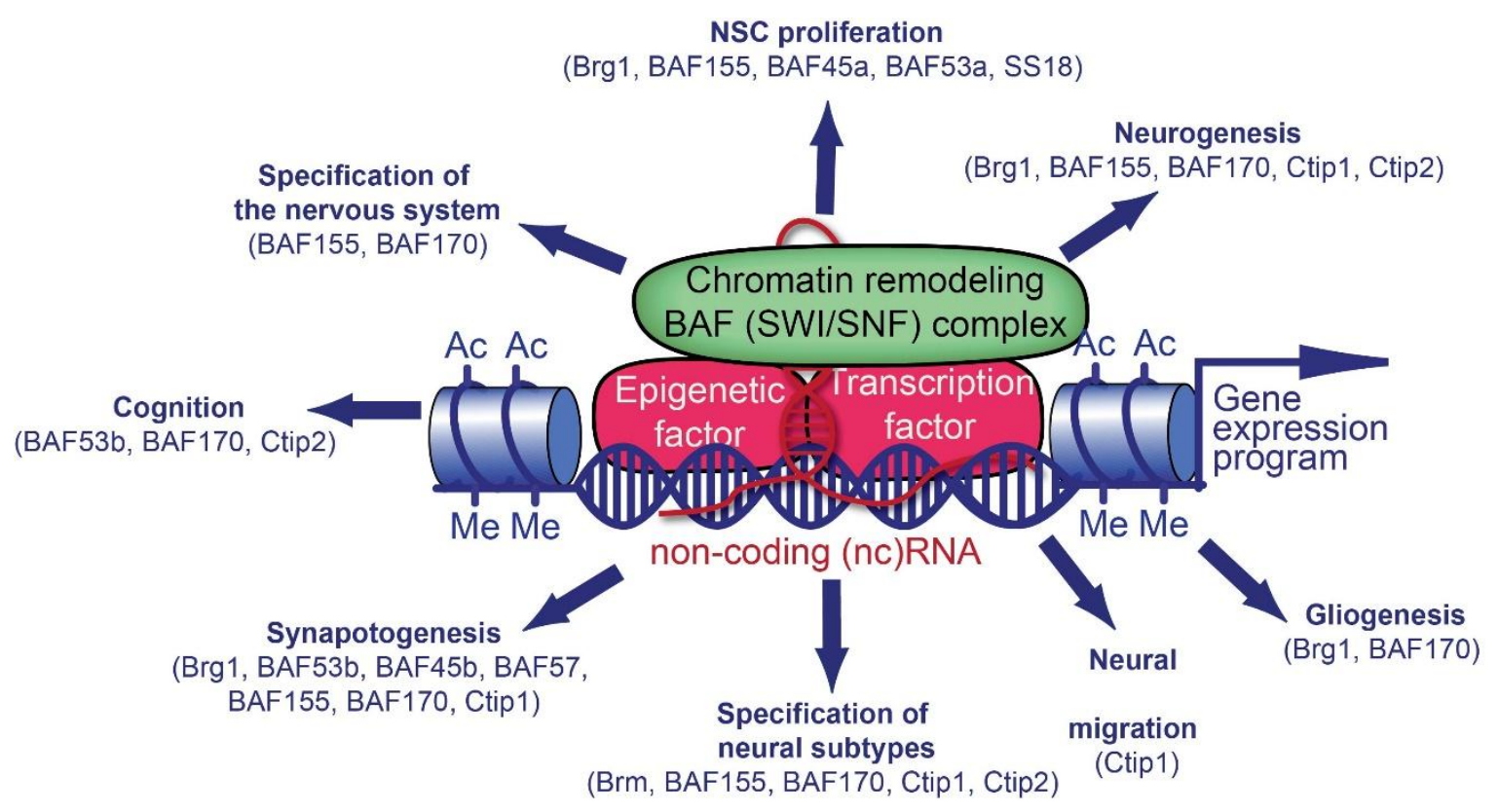

Figure 1.1 Chromatin remodeling BAF (mSWI/SNF) complex in neural development. The BAF complex, epigenetic factors and transcription factors (TF) control gene expression. TFs and ncRNAs bind to specific DNA sequences. The recruitment of BAF complexes and other epigenetic factors on the genome leads to altered epigenetic marks (e.g., histone acetylation, Ac; histone methylation, Me) and chromatin structure in order to activate or repress a specific gene expression program in cell lineages. This figure taken from Sokpor et al. (2017).

Normally, epigenetic modifiers that target chromatin work as a complex machinery to modulate higher-level chromatin configuration to impact many biological processes, including cell renewal, differentiation, motility, maturation, survival and 
reprogramming (Figure 1.1) (Reik, 2007; Boland et al., 2014; Sokpor et al., 2017; Hanna et al., 2018). The outcome of various epigenetic modifications broadly converges on either gene repression or activation. Generally, epigenetic regulators that promote gene expression activation remodel compact chromatin structure to an open or relaxed chromatin. The relaxed chromatin is known to be transcriptionally active because of related increase accessibility by transcription factors (Hirabayashi \& Gotoh, 2010; Juliandi et al., 2010; Coskun et al., 2012; Ronan et al., 2013; Yao et al., 2016; Watson \& Tsai, 2017). The converse is true for transcription repression being caused by chromatin modifiers that render the chromatin compact.

The epigenetic regulators of chromatin structure can be categorized into: covalent and non-covalent chromatin modifiers. Covalent modifiers regulate chromatin via processes including methylation, acetylation, phosphorylation and ubiquitination, whereas non-covalent chromatin modification includes ATP-dependent chromatin remodelers which have been implicated in regulating many developmental processes, including neurodevelopment (Strahl \& Allis, 2000; Neilson et al., 2006; Goldberg et al., 2007; Tran et al., 2013; Narayanan et al., 2015a; Bachmann et al., 2016b; Nguyen et al., 2016; Nguyen et al., 2018).

\subsection{ATP-dependent chromatin modifiers}

The ATP-dependent chromatin remodeling factors are multi-subunits complexes that depend on energy obtained from ATP breakdown to orchestrate detectable alterations in DNA-histone interactions that frequently translate in transcriptional changes to influence cellular developmental processes (Hirabayashi et al., 2009; Yoo \& Crabtree, 2009; Hirabayashi \& Gotoh, 2010; Ho \& Crabtree, 2010; Yao et al., 2016; Albert et al., 2017; Sokpor et al., 2017). Mechanistically, chromatin remodeling involves nucleosomal mobilization that enhances the accessibility of DNA sequences to regulatory proteins that target genomic loci (Reinke \& Hörz, 2003; Bailey et al., 2011).

ATP-dependent chromatin remodeling complexes typically have ATPase subunits that allow them to hydrolyze ATP and to use the generated energy in order to remodel the chromatin structure. The mobilization of chromatin domains to alter DNA access is considered as a general mechanism that defines all ATP-dependent 
chromatin remodelers (Clapier et al., 2017). Based on similarities and differences in their ATPase domains and related subunits, the chromatin remodelers can be further classified into four categories of complexes: INO80/SWR, imitation switch (ISWI), chromodomain helicase DNA-binding (CHD)/Nucleosome Remodeling Deacetylase (NuRD), and switch/sucrose non-fermentable (SWI/SNF) (Flaus et al., 2006).

My study focused on the SWI/SNF complex that have been shown to play indispensable role in embryonic development including neurodevelopment and neuropsychiatric disorders (Sokpor et al., 2017).

\subsection{Biochemical features of the SWI/SNF (BAF) Complex}

The SWI/SNF complex was first identified in yeast to be composed of few subunits (Neigeborn \& Carlson, 1984; Wang et al., 1996a). However, the mammalian orthologs, $\mathrm{mSWI} / \mathrm{SNF}$, or the $\mathrm{Brg} 1 / \mathrm{Brm}$ associated factor (BAF) complex is made up of about 15 subunits totaling about 2 Megadalton (MDa) in size (Lessard et al., 2007; Wu et al., 2007).

The BAF complex is typically found around gene promoters and enhancers, thus making them participate in gene expression programs that orchestrate cell biological processes including cell renewal, specification, differentiation and migration. Like other ATP-dependent chromatin remodelers, the BAF complex is composed of exchangeable ATPase catalytic core(s): either BRM/SWI2 related gene 1 (BRG1) or Brahma (BRM) depending on cell lineage (Neigeborn \& Carlson, 1984; Wang et al., 1996a; Lessard et al., 2007; Wu et al., 2007; Kadoch et al., 2013). The BAF complex also contains other core subunits, including BAF155, BAF170 and BAF47 and variant subunits such as BAF60, BAF100, and BAF 250 that are ubiquitously expressed in the mammalian cell (Phelan et al., 1999; Sokpor et al., 2018). Some of variant subunits are expressed specifically in certain cell lineages such as BAF45A, BAF53A in neural stem cells and BAF45B, BAF53B in neurons (Bachmann, 2016; Lessard, 2007). 
Mechanistically, BAF complex is able to convert condensed chromatin (heterochromatin) to transcriptionally active euchromatin via histone dimer exchanges or nucleosomal mobilization, ejection, and unwrapping (Phelan et al., 1999; Whitehouse et al., 1999; Saha et al., 2002; Gutiérrez et al., 2007; Tang et al., 2010).

Many BAF subunits contain binding domains that allow the BAF complex to interact with DNA and/or histone and regulate gene expression in cell lineage restricted manner. The BAF complex displays variability and specificity in vivo due to combinatorial assembly and switch of its subunits to form complexes with specific remodeling outcomes and gene expression effects on cell fate (Lessard et al., 2007; Wu et al., 2007; Kadoch et al., 2013; Tran et al., 2013; Bachmann et al., 2016a).

\subsection{Regulation of cortical development by the mammalian SWI/SNF (BAF) complex}

During early development of the cerebral cortex, neuroepithelial (NE) cells which initially predominate the germinative zone of the presumptive cortex undergo proliferative (symmetric) division to increase their pool and subsequently switch to differentiative (asymmetric) division to produce the more specialized apical progenitors (radial glial [RG] cells) and pioneer neurons (Martínez-Cerdeño et al., 2006; Kriegstein \& Alvarez-Buylla 2009). The downregulation of tight junctional complexes and the adoption of astroglial fate are characteristic changes that occur during such transformation of NE into RG cells (Mollgøard \& Saunders, 1975; Aaku-Saraste et al., 1997; Hartfuss et al., 2001; Malatesta et al., 2003). Majority of NE cells differentiate to RG cells around embryonic day 12.5 (E12.5) of mouse cortical development (Kriegstein \& Alvarez-Buylla, 2009; Sahara \& O'Leary, 2009). The parent $R G$ cells also known referred to as apical $R G$ cells actively proliferate to increase their population and later exit the cell cycle as other subtypes of apical RG cells or basal progenitors, or as neurons that migrate to make the nascent cortical plate (Florio \& Huttner, 2014). By mid-corticogenesis the developing cortex is populated by diverse neural precursor cells that produce majority of the neurons 
that form the laminae of the cortical plate. The RG cells in the ventricular zone of the developing cortex switch from neurogenic fate to astrocytic progenitor fate to produce astrocytes and in the mouse cortex it starts from E17.5 (Morest, 1970; Schmechel \& Rakic, 1979; Misson et al., 1991).

Many transcriptional and epigenetic factors have been identified to regulate various discrete cortical developmental process including neural progenitor cell specification, proliferation, differentiation, migration and maturation (Sokpor et al., 2017; Elsen et al., 2018). The BAF complex plays critical role in many aspects brain development and function. Specific subunits of the BAF complex have been associate to neurodevelopmental processes, including progenitor proliferation and differentiation, and neuronal migration, maturation and synaptogenesis. As a result, malfunction of the BAF complex have been linked to several neurodevelopmental and neuropsychiatric disorders (Sokpor et al., 2017).

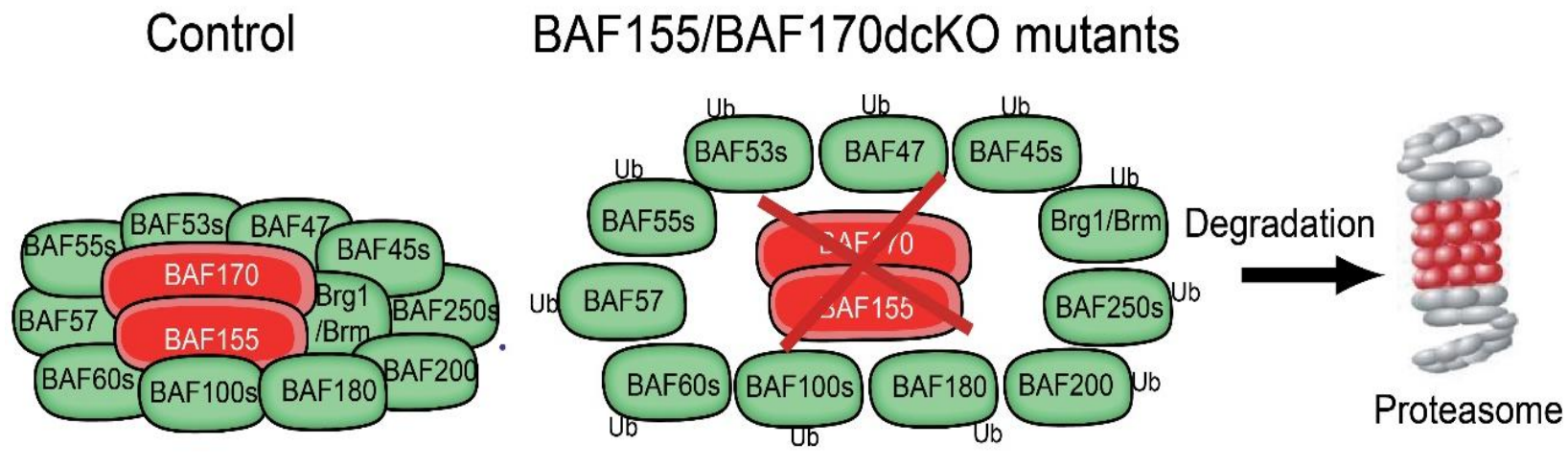

Figure 1.2. Model about the degradation of BAF complexes. Deletion of BAF complex lead to dissociation of the other subunits and their degradation by the protein destruction system. This figure taken from (Narayanan et al. 2015).

In the studies presented here, we developed mouse models to inactivate BAF complex globally in the developing embryo and conditionally in the dorsal telencephalon. The ablation of BAF complex was achieved by deletion of BAF155 and BAF170, leading to dissociation of the other subunits and their degradation by the protein destruction system (Figure 1.2) (Narayanan et al., 2015a). That way, the chromatin remodeling function of the BAF complex is lost in cells with constitutional 
deletion of BAF155 and BAF170. Upon analyzing the BAF complex-deficient mouse embryo, we identified that the Brg1/Brm associated factor plays critical roles in embryogenesis and organ development (Nguyen et al., 2016). Furthermore, we found evidence implicating the regulatory influence of BAF complex on cortical, hippocampal and olfactory epithelium morphogenesis through regulation of neural progenitor proliferation and differentiation (Tran et al., 2013; Bachmann et al., 2016b; Nguyen et al., 2016; Tran et al., 2017; Nguyen et al., 2018).

\section{Aims and general results of the studies}

The studies aimed to clarify the role of BAF complexes in late cortical development and beyond. The studies addressed two major questions: (i) the in vivo validity and reproducibility of the mouse model of inactive BAF complex, and (ii) the implication of loss of BAF complex on cortical organogenesis. To answer these questions we first investigated the role of BAF155 and BAF170 in maintaining the stability of the BAF complex in the entire mouse embryo and specifically in the developing mouse forebrain. Second, we dived into how the BAF complex regulate neurogenesis during late cortical development. Our generated BAF complex mutant model provided a novel and investigative tool to probe into the above mention question in order to confirm our understanding of how the epigenetic regulation by the BAF complex influence cortical development.

The published findings presented in chapter 2 , we identified an indispensable BAF complex function in directing general development of the mouse embryo and profoundly in the early development of the forebrain. Globally, the BAF complex controls the installing of the transcription repressing heterochromatin marks H3K27me2 and H3K27me3 via modulation of the H3K27 demethylases (UTX and JMJD3) enzymatic activity know to control the dynamics of such transcription repression marks (Nguyen et al. 2018; Nguyen et al. 2016; Narayanan et al. 2015). As

a result, deletion of the BAF complex resulted in marked upregulation of H3K27me2 
and H3K27me3 in many organs in the mouse embryo, including the brain leading to developmental disturbance.

In chapter 3, we showed the mechanistic details of how the BAF complex regulate cortical development. We found that the BAF complex functions as both repressors and activators to control the epigenetic landscape and related corticogenic gene expression programs in late cortical development. Specifically, BAF complexes ablation led to H3K27me3-linked repression of neuronal differentiation-associated genes, with simultaneous H3K4me2-mediated enhancement of proliferation-related genes through Wnt signaling de-repression. Interestingly, loss of BAF complex encourage proliferation of NE-like neural stem cells and apparently prolonged their transformation into $\mathrm{RC}$ cells. Altogether, loss of BAF complex functionality resulted in impaired neural progenitor proliferation and differentiation, and had a Wnt-dependent impact on proper cerebral cortex (neocortex and hippocampus) development. 


\section{Chapter 2: Epigenetic regulation by BAF (mSWI/SNF) chromatin remodeling complexes is indispensable for embryonic development}

Huong Nguyen ${ }^{1 \#}$, Godwin Sokpor ${ }^{1 \#}$, Linh Pham ${ }^{1}$, Joachim Rosenbusch ${ }^{1}$, Anastassia Stoykova $^{2,3}$, Jochen F. Staiger ${ }^{1,3}$, and Tuoc Tran ${ }^{1,3}$

Personal contributions: I and G.S. were involved in characterization of dcKO phenotypes, data analysis and preparation of the manuscript. L.P. and J.R. contributed to histological analyses; T.T. supervised, and wrote the manuscript; J.F.S., A.S. offered suggestions for the study.

\# Equally contributed authors

\subsection{Abstract}

The multi-subunit chromatin-remodeling SWI/SNF (known as BAF for $\mathrm{Brg} / \mathrm{Brm}$-associated factor) complexes play essential roles in development. Studies have shown that the loss of individual BAF subunits often affects local chromatin structure and specific transcriptional programs. However, we do not fully understand how BAF complexes function in development because no animal mutant had been engineered to lack entire multi-subunit BAF complexes. Importantly, we recently reported that double conditional knock-out (dcKO) of the BAF155 and BAF170 core subunits in mice abolished the presence of the other BAF subunits in the developing cortex. The generated $d c K O$ mutant provides a novel and powerful tool for investigating how entire BAF complexes affect cortical development. Using this model, we found that BAF complexes globally control the key heterochromatin marks, $\mathrm{H} 3 \mathrm{~K} 27 \mathrm{me} 2$ and -3 , by directly modulating the enzymatic activity of the H3K27 demethylases, Utx and Jmjd3. Here, we present further insights into how the scaffolding ability of the BAF155 and BAF170 core subunits maintains the stability of BAF complexes in the forebrain and throughout the embryo during development. Furthermore, we show that the loss of BAF complexes in the above-described model up-regulates $\mathrm{H} 3 \mathrm{~K} 27 \mathrm{me} 3$ and impairs forebrain development and embryogenesis. These findings improve our understanding of epigenetic 
mechanisms and their modulation by the chromatin-remodeling SWI/SNF complexes that control embryonic development.

\subsection{Introduction}

Embryogenesis and organogenesis are determined by the combined effects of myriad developmental events. In recent years, we have made substantial advances in understanding how embryonic development is regulated (Ho \& Crabtree, 2011; Kojima et al., 2014). The early development are coordinated by different molecular programs, in which epigenetic and chromatin-related controls are known to play crucial roles (Ho \& Crabtree, 2011). Epigenetic regulation, which modulates the chromatin structure without altering the DNA sequence, has profoundly heritable influences on transcriptional programs (Heard \& Martienssen, 2014). These changes in chromatin organization activate or repress gene expression programs either globally or locally, and may thus shape specific developmental events. Epigenetic mechanisms and chromatin regulation influence the ability of transcription factors (TFs) to access regulatory elements in their target genes. This occurs primarily via histone modification (Goldberg et al., 2007) or the action of ATP-dependent chromatin remodeling complexes, such as SWI/SNF (BAF) complexes (Wen et al., 2009; MuhChyi et al., 2013; Narlikar et al., 2013; Ronan et al., 2013). In addition, recent studies have shown that DNA methylation (Wu \& Zhang, 2014) and long non-coding RNA (IncRNA)-based mechanisms (Bohmdorfer \& Wierzbicki, 2015) also contribute to the complexity of epigenetic regulation during development.

The types of covalent histone modification include histone acetylation, methylation, ubiquitination and phosphorylation (Strahl \& Allis, 2000; Goldberg et al., 2007). Histone modification (epigenetic marks) is catalyzed by two enzyme classes: histone writers (e.g., histone acetyltransferases, methyltransferases, kinases, and ubiquitin ligases) and histone erasers (e.g., histone deacetylases, demethylases, phosphatases, and deubiquitinases). The mis-regulation of histone writers and erasers will typically alter the epigenetic program and have profound effects on development (Strahl \& Allis, 2000; Goldberg et al., 2007). 
A number of non-covalent, energy-dependent chromatin remodeling complexes modulate the dynamicity of chromatin structures. Among them, the SWI/SNF complexes are the best characterized in both development and disease. Mammalian SWI/SNF (BAF) complexes are made up of two switchable ATPase subunits (Brg1 or Brm), core subunits (BAF47, BAF155, and BAF170) and a variety of lineage-specific subunits (Lessard et al., 2007; Ho et al., 2009b; Kadoch et al., 2013; Ronan et al., 2013). The Brg1 and Brm ATPases hydrolyze adenosine triphosphate (ATP) and utilize the obtained energy to alter chromatin (nucleosome) structures, thereby modulating cellular processes such as gene expression (Hirschhorn et al., 1992; Laurent et al., 1993; Phelan et al., 1999). The various subunits (at least 15 have been identified) are capable of undergoing combinatorial assembly (Wang et al., 1996b; Ronan et al., 2013), yielding hundreds of distinct BAF complexes that can direct specific transcriptional events during development in vivo. The exceptional diversity of BAF complexes allows them to have functional specificity in biological processes. To investigate the roles of BAF complexes in development, researchers have focused on phenotypic analyses of model animals harboring mutations in single BAF subunits (Ko et al., 2008; Ho \& Crabtree, 2011; Narayanan \& Tran, 2014). However, although BAF complexes are known to play essential roles in development, studies using knock-out mouse models for individual BAF subunits have yielded incomplete information regarding the functions of these complexes.

While the epigenetic machinery and chromatin-remodeling complexes are known to play essential roles in development, we know little about how they interact to coordinate developmental processes during embryogenesis and organogenesis. Recently, our group developed cortex-specific BAF155/BAF170CKO mouse mutant and showed that BAF complexes did not form in the cortices of these mice. We further showed that the known BAF subunits undergo proteasome-mediated degradation in the developing cortices of these mutants. Finally, we found that, during corticogenesis, BAF complexes globally control key heterochromatin marks (H3K27me2/3) by directly interacting with and modulating the enzymatic activity of the H3K27 demethylases. Here, we discuss these recent discoveries (Narayanan et al., 2015) and present 
additional evidence suggesting that the BAF155 and BAF170 core subunits cooperate to stabilize the BAF complex and maintain the global level of H3K27me3 both in the developing forebrain and throughout the embryo. Our new findings indicate that BAF complexes act as key regulators of embryogenesis.

\subsection{Results and Discussion}

\subsubsection{BAF155 and BAF170 are indispensable for brain development and embryogenesis}

By employing cortex-specific conditional mouse mutagenesis, we showed that the dual loss of the BAF155 and BAF170 subunits in double conditional knock-out $(d c K O)$ mutants severely perturbed the growth of cortical structures, blocked the proliferation, differentiation and cell-cycle progression of cortical progenitors, and triggered a massive increase in the number of apoptotic cells (Narayanan et al., 2015). To further investigate how the loss of both BAF155 and BAF170 affects forebrain development, we generated forebrain-specific BAF155 and BAF170 dcKO mice by crossing mice floxed for BAF155 (Choi et al., 2012) and

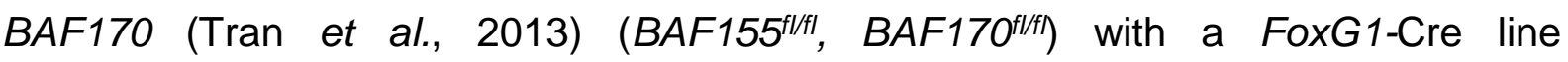
(Hebert \& McConnell, 2000). In FoxG1-Cre mice, the Cre-recombinase is driven in all telencephalic cells [including those of the cortex (Cx) and basal ganglia (BG)], but not in other parts of the brain [e.g., in the diencephalon (Di)] (Hebert \& McConnell, 2000). Remarkably, we found that the dcKO_FoxG1-Cre mutants completely lacked all telencephalic structures at E16.5 (Narayanan et al., 2015). This indicated that the expressions of BAF155 and BAF170 are required for brain development. 


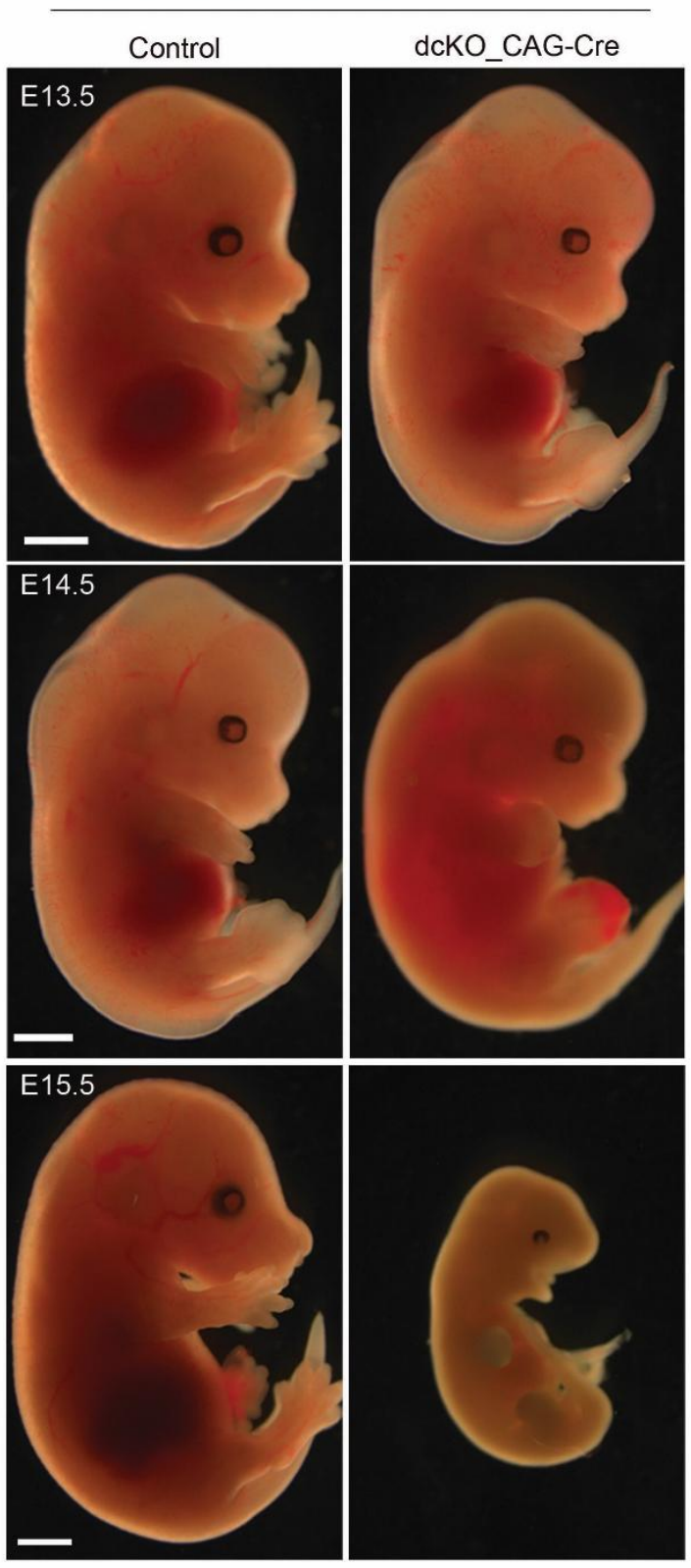

Figure 2.1. The expressions of BAF155 and BAF170 are indispensable for embryonic development: dcKO_CAG-Cre embryos treated with TAM at E9.5 remained alive and showed roughly preserved morphology at E13.5, but thereafter died between E14.5 and E15.5. Scale bars $=1000 \mu \mathrm{m}$.

To address whether BAF155 and BAF170 are essential for embryogenesis, we generated and analyzed a line harboring a full dcKO_CAG-Cre mutant with the tamoxifen (TAM)-inducible ubiquitous deleter, CAG-Cre line (Hayashi \& McMahon, 2002) (Figure 2.1). The dcKO_CAG-Cre mutants were injected with either TAM or corn oil (vehicle solution, control) at E9.5. Following TAM induction, we observed Cre-recombinase activation in all cells of the body (Hayashi \& McMahon, 2002). 
The mutants died between E14.5-E15.5, and exhibited a severe developmental retardation (Figure 2.1). Together, these results show that the expressions of BAF155 and BAF170 are critical for determining overall embryogenesis, including the formations of the forebrain and cortex.

\subsubsection{BAF155 and BAF170 control the stability of BAF complexes in both cultured cells and embryos}

Hundreds of distinct BAF complexes are predicted to form in vivo by the combinatorial assembly of at least 15 identified BAF subunits (Ho \& Crabtree, 2011). The functional specificity of a BAF complex is believed to reflect the composite surfaces of its integrated subunits, which are essential for the ability of these complexes to target the genome and interact with transcriptional factors (TFs), co-activators, co-repressors, and signaling pathways (Ho \& Crabtree, 2011). We recently reported that BAF155 and BAF170 act as scaffolding subunits and are required to ensure the stability of the entire BAF complex in the developing cortex (Narayanan et al., 2015). The loss of BAF155 and BAF170 in cortex-specific dcKO mutants leads to the dissociation of all other BAF subunits from the complex. The free BAF subunits are subsequently ubiquitinated and degraded by the proteasome system.

In an effort to extend our analysis to other parts of the brain, we examined the expression levels of various BAF subunits (e.g., Brg1, Brm, BAF47, BAF60, and BAF250) following the loss of BAF155/BAF170 in telencephalon of dcKO_FoxG1-Cre embryos (Figure 2.2). Consistent with the Cre-recombinase activity in the $\mathrm{Cx}$ and $\mathrm{BG}$ of dcKO_FoxG1-Cre mice, there was no detectable expression of BAF155 or BAF170 in these structures. In contrast, their expression levels were preserved in the Di, where Cre is inactive (Figure 2.2 A, B). Similar to the reported effects in cortical tissues (Narayanan et al., 2015), the loss of BAF155 and BAF170 in the telencephalon abrogated the expression of all BAF subunits throughout this structure, including in the $\mathrm{BG}$ (Figure 2.2C-G). To investigate whether both BAF155 and BAF170 are required to stabilize BAF complexes throughout the embryo, the expression of BAF subunits was examined in ubiquitously inducible dcKO_CAG-Cre embryos with global loss of BAF155/BAF170 (Figure 2.3). These dcKO_CAG-Cre mutants were injected with either TAM or corn oil (vehicle solution, as control) at E9.5 and analyzed at E13.5, when the mutants were still viable. Following treatment with TAM, the expression levels of BAF155 and BAF170 were considerably ablated (Figure 2.3A-D). 
Moreover, the expression levels of the tested BAF subunits (Brg1, Brm, BAF47, and BAF250) were severely diminished throughout the dcKO_CAG-Cre embryos, as compared to controls (Figure 2.3E-L). These findings suggest that BAF155 and BAF170 are required to maintain the expression levels of BAF subunits in living animals.
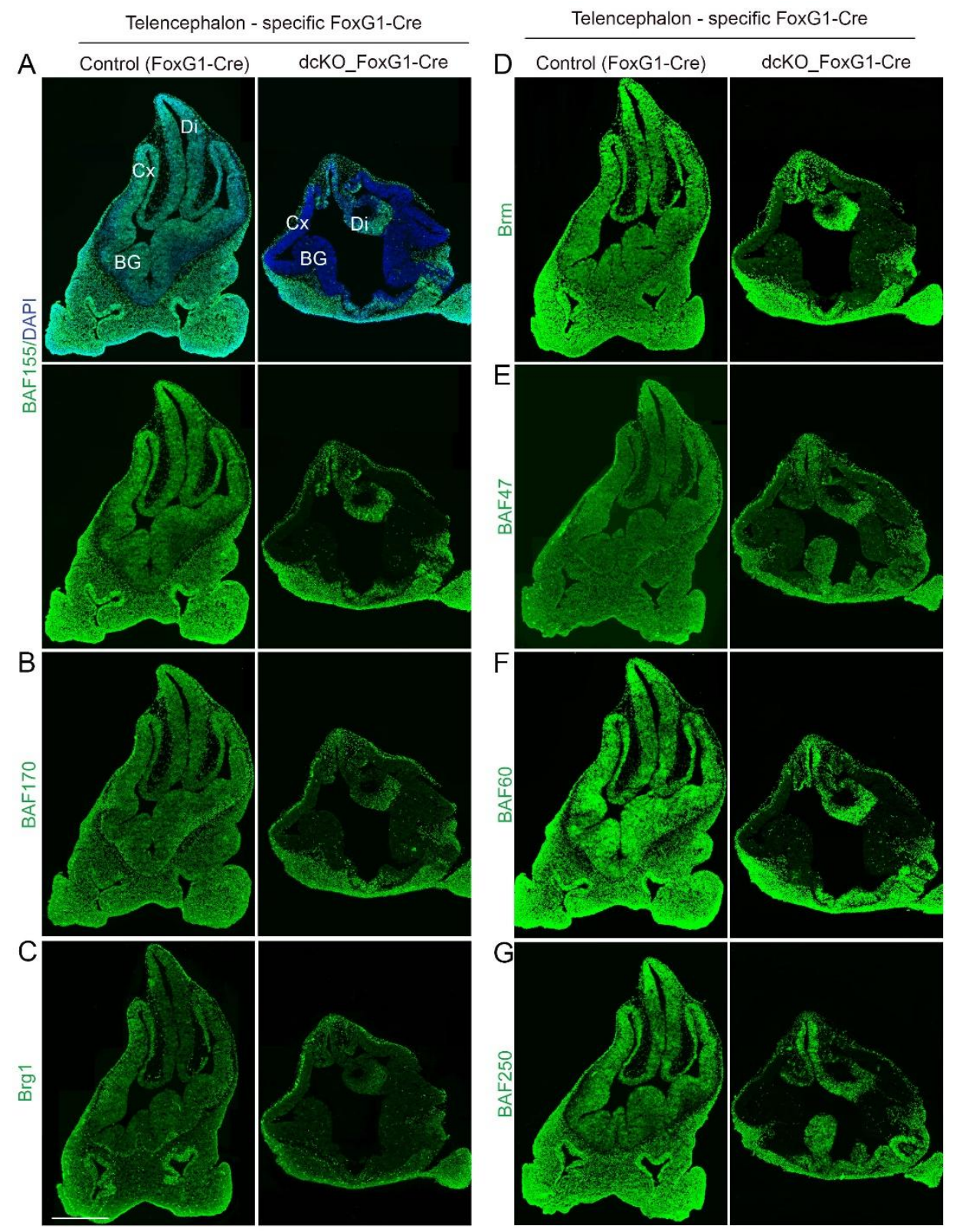

Figure 2.2. Expression of BAF subunits in telencephalon-specific dcKO_FoxG1-Cre mutants. (A-G) Images show immunohistochemical $(\mathrm{IHC})$ analyses for various core subunits of BAF complexes, including BAF155 (A), BAF170 (B), Brg1 (C), Brm (D), BAF47 (E), BAF60 (F), and BAF250 (G), in the forebrains of dcKO_FoxG1-Cre mutants at E11.5. The indicated BAF subunits are not detected in the BAF155/BAF170-knockout telencephalon. Scale bars $=500 \mu \mathrm{m}$. Abbreviations: $\mathrm{Cx}$, cortex; BG, basal ganglia; and Di, diencephalon. 
E13.5 (TAM: E9.5)

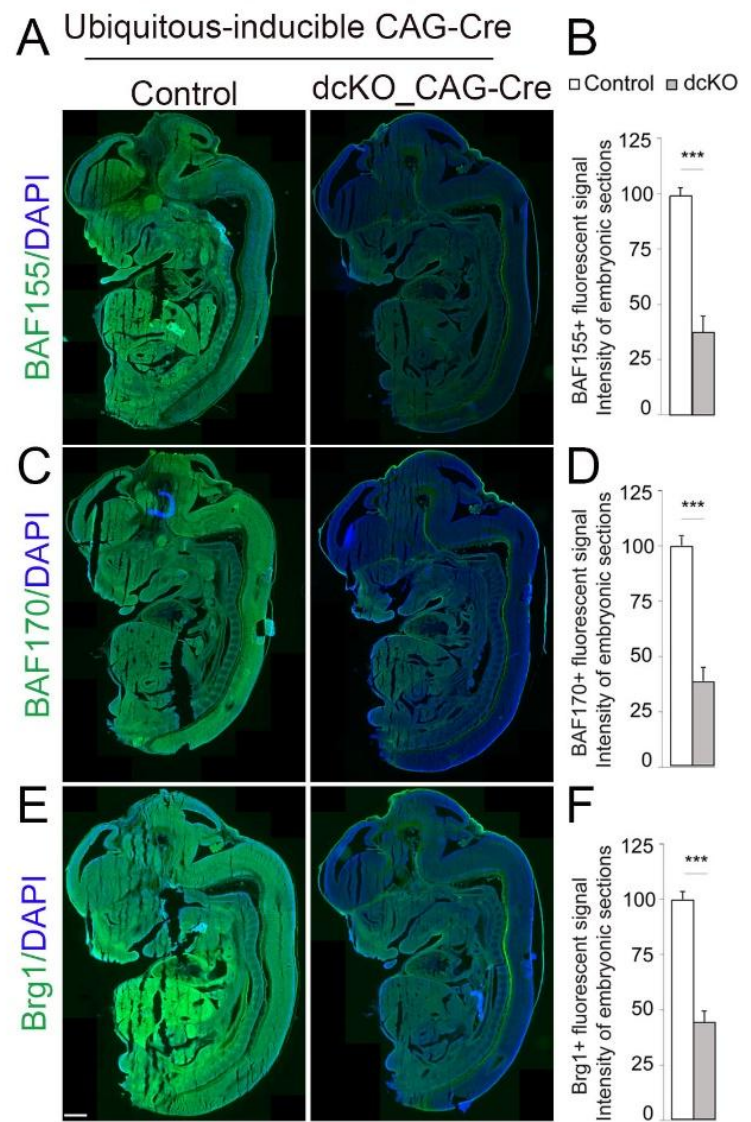

E13.5 (TAM: E9.5)

G Ubiquitous-inducible CAG-Cre $\mathrm{H}$

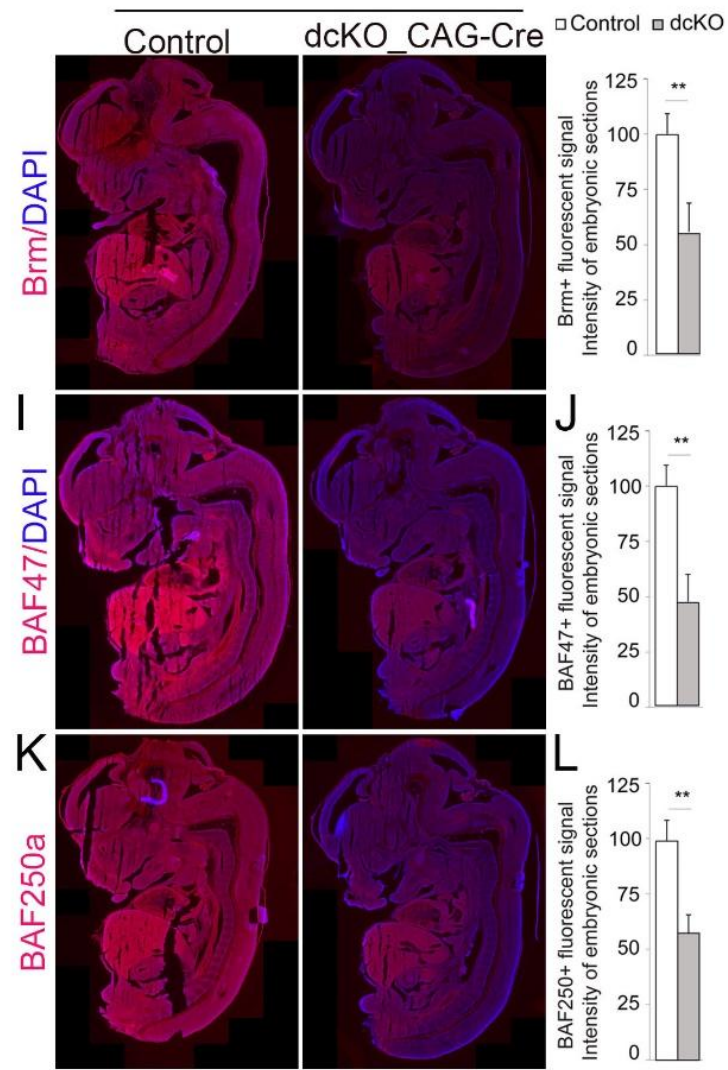

Figure 2.3. Expression of BAF subunits in embryos of TAM-inducible full dcKO_CAGCre mutants. (A/C/E/G//K) E13.5 dcKO_CAG-Cre mutant embryos were treated with TAM at $E 9.5$, and whole-embryo sections were immunostained with antibodies against BAF155 (A), BAF170 (C), Brg1 (E), Brm (G), BAF47 (I), and BAF250a (K).(B/D/F/H/J/L) Quantifications of fluorescent signal intensities obtained from the sections described $(A / C / E / G / / / G)$ (see also Table $\mathrm{S} 1$ for statistical analysis). The results revealed that the protein expression levels of BAF155 and BAF170 were reduced throughout the TAM-treated dcKO_CAG-Cre mutant embryos, confirming the double knockdown of BAF155/BAF170. The expression levels of the other tested BAF subunits were also diminished in mutant embryos compared to controls. Scale bars $=500 \mu \mathrm{m}$. 
In different tissues and cell lineages, BAF155 is highly expressed in proliferating stem/progenitor cells but generally down-regulated upon differentiation (Yan et al., 2008; Ho et al., 2009a; Tran et al., 2013). Conversely, little BAF170 is expressed in stem/progenitor cells (e.g., embryonic stem cells, or ESCs) and at higher levels in differentiated cells (e.g., neurons) (Yan et al., 2008; Ho et al., 2009a; Tran et al., 2013). We hypothesized that although only low expression levels are detected for BAF170 in proliferating ESCs and for BAF155 in post-mitotic neurons, this expression is necessary and sufficient to stabilize the embryonic stem cell (es)BAF and neuronal (n)BAF complexes. Indeed, when we derived ESC lines from blastocysts and primary neurons from forebrains (both representing the dcKO_CAG-Cre genotype), we found that the depletion of BAF155 and BAF170 in these cultured cells led to the loss of BAF subunit expression at the protein level (Narayanan et al., 2015).

These results collectively indicate that the knockout of BAF155/BAF170 in dcKO mutants eliminates the presence of known BAF complex subunits both in vitro and in vivo. Thus, the $d c K O$ mutants provide a potent tool for investigating the roles of entire BAF complexes during development.

\subsubsection{The loss of BAF complexes induces the accumulation of H3K27me2/3- marked heterochromatin}

Previous studies suggested that the loss of individual BAF subunits has a local (not global) influence on chromatin marks (Ho et al., 2011; Tran et al., 2013). However, when we examined epigenetic marks in cortex-specific dcKO_Emx1-Cre mice, which lacked entire BAF complexes, we observed a global reduction in euchromatin along with increased H3K27me2/3 and decreased H3K9Ac in the developing cortex during both embryonic and perinatal stages, as assessed by assays such as ChIP-Seq, immunohistochemistry, and western blotting (Narayanan et al., 2015). Thus, our data showed for the first time that the presence of BAF complexes is needed to maintain the balance between global repression and local activation of epigenetic programs during cortical development (Narayanan et al., 2015). 
The intriguing observation that BAF complexes are lost from the telencephalonspecific dcKO_FoxG1-Cre and inducible full dcKO_CAG-Cre mutants prompted us to study how this BAF155/BAF170 loss-of-function affects the H3K27me3 repressive mark. We performed western blotting (WB) on telencephalic tissue lysates from E11.5 dcKO_FoxG1-Cre mutants using an antibody against H3K27me3. Similar to our observation in cortical tissues, we found that the loss of BAF155 and BAF170 increased the level of H3K27me3 in telencephalon (Figure 2.4A/C). Likewise compared to control (non-injected) embryos, the H3K27me3 level was augmented in E13.5 dcKO_CAG-Cre embryos that had been injected with TAM at E9.5 (Figure 2.4B/C).

H3K27me2 and -3 are chromatin modifications that have been linked to the down-regulation of gene expression (Cao et al., 2002; Pereira et al., 2010). Thus, the massive enhancement of H3K27me3 in the dcKO mutants would be expected to trigger obvious repression of gene expression. Indeed, gene expression profiling of developing cortices from $d c K O$ mutants revealed that most of the transcripts were down-regulated, with only a few showing up-regulation (Narayanan et al., 2015). Remarkably, BAF complexes were found to positively regulate most of the genes that are repressed by the H3K27 methyltransferase, Ezh2 (Pereira et al., 2010; Narayanan et al., 2015). 
A

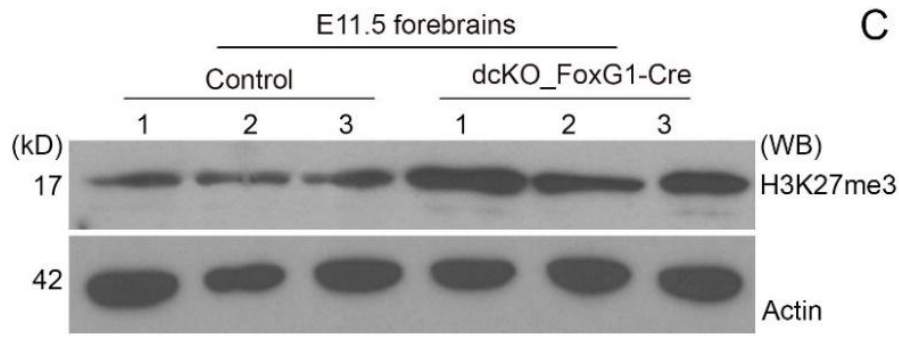

B

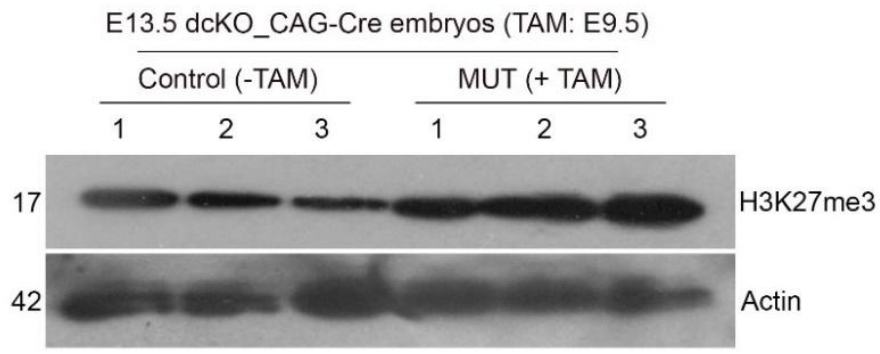

D
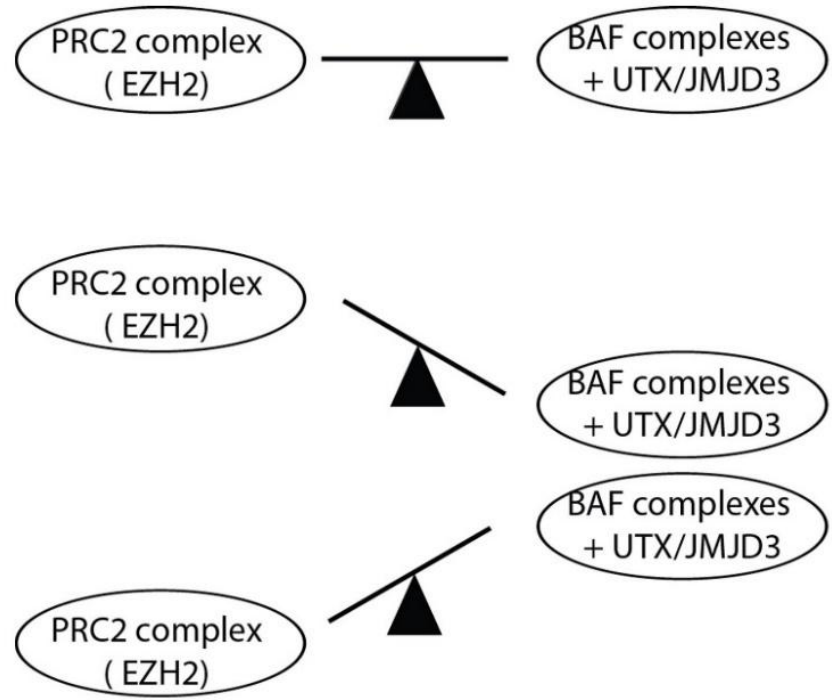

C

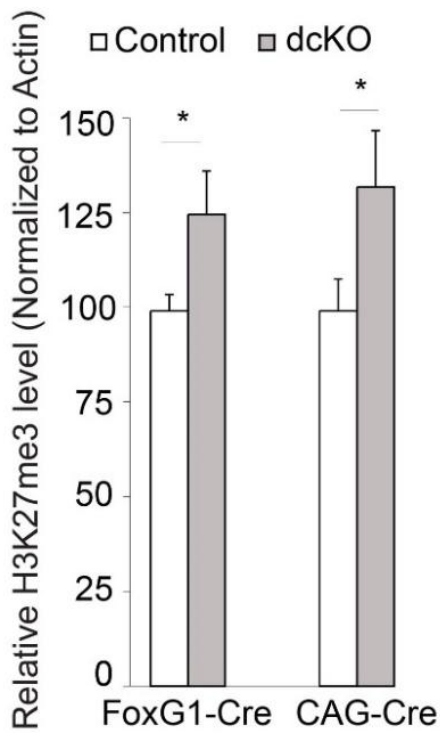

Figure 2.4. BAF complexes control the level of H3K27me3 in the brain and whole embryo

during development. (A) WB analysis of E11.5 telencephalons from telencephalon-specific dcKO_FoxG1-Cre mutants revealed that the lost expressions of BAF155 and BAF170 elevated the level of H3K27me3. (B) dcKO_CAG-Cre embryos treated with TAM at E9.5 showed up-regulation of $\mathrm{H} 3 \mathrm{~K} 27 \mathrm{me} 3$ at E13.5, compared to untreated control embryos. (C) Densitometric quantification of the WB bands shown in (A and B; see also Table S2 for statistical analysis). (D) Schematic indicating how altered levels of H3K27me2/3 demethylases (UTX/Kdm6a and JMJD3/Kdm6b), BAF complexes, and the H3K27 methyltransferase Ezh2 subunit of the PRC2 complex collectively modulate histone methylation, developmental defects and diseases (e.g., tumorgenesis). 
To directly examine the apparent opposing activity of BAF complexes and the Ezh2 subunit of the PRC2 complex, we treated dcKO mutants with an Ezh2 inhibitor and examined gene expression in developing cortex. We found that inhibition of the H3K27 methyltransferase partially rescued the expression of certain BAF-complex target genes (Narayanan et al., 2015). In mechanistic terms, our results suggested that this process involves binding of the BAF155 and BAF170 core subunits of the BAF complex to the JmjC domains of UTX/Kdm6a and JMJD3/Kdm6b, which are required for the $\mathrm{H} 3 \mathrm{~K} 27 \mathrm{me} / 3$ demethylase activities of these proteins (Narayanan et al., 2015).

Ezh2 (or PRC2) (Cao et al., 2002; Shen et al., 2008; Pereira et al., 2010) and UTX/Kdm6a/JMJD3/Kdm6b (Agger et al., 2007; De Santa et al., 2007; Hong et al., 2007; Jepsen et al., 2007; Lan et al., 2007; Lee et al., 2007; Xiang et al., 2007) are the only enzymes known to methylate and demethylate H3K27, respectively. These enzymes play essential roles in development and diseases by modulating gene expression programs through changes in the methylation of H3K27. Studies have shown that homozygous-null Ezh2 mutants die prior to completing gastrulation, conditional loss of maternal Ezh2 results in severe growth retardation among neonates (Erhardt et al., 2003; Puschendorf et al., 2008), and EZH2 overexpression causes tumorigenesis (Varambally et al., 2008; Takawa et al., 2011). Phenotypic analysis revealed that mouse embryos $d c K O$ for Utx and Jmjd3 (which encode the H3K27 demethylases) exhibit lethality at mid-gestation. Moreover, the expression levels of Jmjd3 and Utx are significantly decreased in several types of primary tumors (Agger et al., 2009). The BAF complexes, which we identified as important cofactors of the H3K27 demethylases, are known to be key players in development (Ko et al., 2008; Ho \& Crabtree, 2011; Narayanan \& Tran, 2014; Narayanan et al., 2015) and tumor suppression (Ko et al., 2008; Wu, 2012; Helming et al., 2014; MasliahPlanchon et al., 2015). Thus, any alteration in the balance among the BAF complexes, H3K27 demethylases, and methyltransferase will result in severe developmental defects and/or diseases such as cancer (Figure 2.4C) (Shi, 2007; Pedersen \& Helin, 2010; Ho \& Crabtree, 2011; Kadoch et al., 2016). 


\subsection{Conclusion}

We herein present evidence suggesting that BAF155 and BAF170 act as scaffolding subunits to maintain the stability of BAF complexes. The loss of BAF complexes in BAF155/BAF170 double mutants is associated with severe defects in global epigenetic and gene expression programs during cortical development (Narayanan et al., 2015) and embryogenesis (this study). Our results further suggest that manipulation of the endogenous expression and activity levels of the chromatinremodeling BAF complexes, the H3K27me2/3 demethylases (UTX/Kdm6a and JMJD3/Kdm6b), and the H3K27me2/3 methyltransferase (polycomb repressive complex 2) might enable to alter global gene expression programs. The crosstalk between BAF complexes and epigenetic factors revealed herein may shed light on how cells acquire their fates. This work could thus contribute to the establishment of protocols aimed at differentiating specific lineages from pluripotent cells and/or treating diseases.

\subsection{Materials and Methods}

\subsubsection{Transgenic mice}

Floxed BAF155 (Choi et al., 2012), floxed BAF170 (Tran et al., 2013), FoxG1-Cre (Hebert \& McConnell, 2000) and CAG-Cre (Hayashi \& McMahon, 2002) mouse lines were kept in a C57BL6/J background. All animal research was conducted in accordance with the local regulations for animal protection.

\subsubsection{Immunohistochemistry (IHC) and Western blotting (WB)}

IHC and WB were performed as previously described (Tran \& Stoykova, 2008; Tran et al., 2009). The following polyclonal (pAb) and monoclonal (mAb) primary antibodies used in this study were obtained from the indicated commercial sources: Brg1 rabbit pAb (Santa Cruz), Brg1 mouse mAb (Santa Cruz), Brm mouse mAb 
(BD Biosciences), Brm rabbit pAb (Abcam), BAF250 mouse mAb (Sigma), BAF170 rabbit pAb (Bethyl), BAF170 rabbit pAb (Sigma), BAF155 rabbit pAb (Santa Cruz), BAF155 mouse mAb (Santa Cruz), BAF60a mouse mAb (BD Biosciences), GAPDH rabbit pAb (Santa Cruz), B-actin rabbit pAb (Sigma), and H3K27me3 rabbit pAb (Upstate). The utilized secondary antibodies included peroxidase-conjugated goat anti-rabbit $\lg$ (1:10,000; Covance), peroxidase-conjugated goat anti-mouse IgG (1:5000; Covance), and Alexa 488- or Alexa 568-conjugated IgG (various species, 1: 400; Molecular Probes).

\subsubsection{Imaging and quantitative and statistical analyses}

Imaging was performed with an Axio Imager M2 (Zeiss) with a Neurolucida system (Version 11; MBF Bioscience) and confocal fluorescence microscopes (TCS SP5; Leica). Pictures were analyzed further with Adobe Photoshop.

Densitometric quantification of WB bands and quantitative analysis of $\mathrm{IHC}$ signal intensities were performed using the ImageJ software. Statistical analyses were carried out using the Student's t-test. The results are presented as the mean \pm SEM. 


\section{Chapter 3: Epigenetic Regulation by BAF Complexes Limits Neural Stem Cell Proliferation by Suppressing Wnt Signaling in Late Embryonic Development}

Huong Nguyen ${ }^{1, ~ \#, ~ C e m i l ~ K e r i m o g l u ~}{ }^{2,6}$ \#, Mehdi Pirouz ${ }^{3,7}$ Linh Pham ${ }^{1}$, Kamila A. Kiszka ${ }^{1,4}$, Godwin Sokpor ${ }^{1}$, M. Sadman Sakib ${ }^{2,6}$, Joachim Rosenbusch ${ }^{1}$, Ulrike Teichmann ${ }^{3}$, Rho H. Seong ${ }^{5}$, Anastassia Stoykova ${ }^{3,4}$, Andre Fischer ${ }^{2,6}$, Jochen F. Staiger ${ }^{1,4}$, and Tuoc $\operatorname{Tran}^{1,4}$ *

Personal contributions: I was involved in performance of most histological analyses of dcKO phenotypes, data analysis and preparation of the manuscript.

C.K. and A.F. generated RNA-Seq and ChIP-Seq data; M.P. performed the protein-protein interaction study; L.P., G.S. and J.R. contributed to histological analyses; K.A.K. characterized hGFAP-Cre_ROSA-dtTOM mouse line; C.K. performed ChIP-qPCR; M.S.S. performed qPCR; J.F.S. R.H.S, U.T and A.S. provided research tools, transgenic lines, and contributed to discussions; T.T. conceived, supervised, and wrote the manuscript; J.F.S., A.S., and A.F. offered suggestions for the study.

\# Equally contributed authors

\subsection{Summary}

During early cortical development, neural stem cells (NSCs) divide symmetrically to expand the progenitor pool, whereas in later stages, NSCs divide asymmetrically to self-renew and produce other cell types. The timely switch from such proliferative to differentiative division critically determines progenitor and neuron numbers. However, the mechanisms that limit proliferative division in late cortical development are not fully understood. Here, we show that the BAF (mSWI/SNF) complexes restrict 
proliferative competence and promote neuronal differentiation in late corticogenesis. Inactivation of BAF complexes leads to H3K27me3-linked silencing of neuronal differentiation-related genes, with concurrent H3K4me2-mediated activation of proliferation-associated genes via de-repression of Wnt signaling. Notably, the deletion of BAF complexes increased proliferation of neuroepithelial cell-like NSCs, impaired neuronal differentiation and exerted a Wnt-dependent effect on neocortical and hippocampal development. Thus, these results demonstrate that BAF complexes act as both activators and repressors to control global epigenetic and gene expression programs in late corticogenesis.

\subsection{Introduction}

During vertebrate cerebral cortex development, neural stem cells (NSCs) undergo two types of temporally regulated cell division modes to generate distinct neural cell types. During early corticogenesis in mice (embryonic day 8.5 to 12.5 [E8.5-E12.5]), NSCs, also called neuroepithelial cells (NEs), mainly divide symmetrically to proliferate and expand their population (Gotz \& Huttner, 2005; Dehay \& Kennedy, 2007; Kriegstein \& Alvarez-Buylla, 2009; Martynoga et al., 2012; Tran et al., 2014). At the onset of neurogenesis (E10.5), NEs differentiate into mature NSCs, also termed radial glial progenitors (RGs), which start to express astroglial markers (Hartfuss et al., 2001). This process coincides with the loss and appearance of tight and adherens junctional complexes respectively in the ventricular zone (VZ) (Aaku-Saraste et al., 1996; Sahara \& O'Leary, 2009). Later, RGs primarily divide asymmetrically to produce an $R G$ to maintain the proliferative pool, and either an excitatory neuron or a basal progenitor. Delayed RG differentiation from NEs causes aberrant neurogenesis (Sahara \& O'Leary, 2009), yet factors that are required to suppress NE fate in late corticogenesis to ensure a balance between NSC proliferation and neuronal differentiation are unknown.

The temporal relationship and intricate balance between proliferative symmetric and neurogenic asymmetric divisions in the VZ of the cortex is controlled by diverse 
signaling pathways. Among these, Wnt/ $/$-catenin signaling has been extensively investigated for its role in proliferative symmetric division (Chenn \& Walsh, 2002). For example, elevation of Wnt signaling through overexpression of $\beta$-catenin massively enhanced cortical NSC proliferation (Chenn \& Walsh, 2002). Interestingly, a recent study revealed irreversibility of the progression from proliferative to neurogenic division modes, thus implicating a default program in NSCs for division-mode transition during corticogenesis (Gao et al., 2014). As regulators of the spatiotemporal expression of developmental genes, epigenetic and chromatin regulatory mechanisms have been proposed to contribute to establishing the proliferative and differentiation competence of NSCs (Hirabayashi \& Gotoh, 2010; Yao et al., 2016).

To investigate the possible involvement of the chromatin remodeling BAF (mSWI/SNF) complexes in this process, we applied a conditional deletion approach through double-knockout (dcKO) of the BAF155 and BAF170 subunits, which eliminate the entire BAF complex during the late cortical neurogenesis in transgenic mice. In the absence of BAF complexes, transcriptional profiling and epigenetic analyses revealed an enrichment of down-regulated RG (astroglial, adherens junctions)-, and neuronal differentiation-related genes, with both gene groups showing increased H3K27me3 repressive marks. In contrast, upregulated genes with increased $\mathrm{H} 3 \mathrm{~K} 4 \mathrm{me} 2$ active marks were predominantly involved in the regulation of NE cell fate (e.g. tight junction feature), proliferation, cell cycle, and Wnt signaling-related pathways. The results of this study suggest that BAF complexes exert genome-wide control on both active H3K4me2 and repressive H3K27me3 marks during late cortical development by directly interacting with the corresponding $\mathrm{H} 3$ demethylases and regulating their activity. Phenotypically, we found that deletion of BAF complexes during late cortical neurogenesis leads to dysgenesis of the upper cortical layers and the hippocampal formation. These perturbations were rescued by inhibition of $\mathrm{Wnt} / \beta$-catenin signaling. Together, these observations provide new insights into distinct epigenetic regulatory mechanisms mediated by chromatin-remodeling BAF complexes as a key factor that suppresses the proliferative competence of NSCs during late cortical development. 


\subsection{Results}

\subsubsection{Loss of BAF complexes causes a genome-wide increase in the level of both active and repressive epigenetic marks at distinct loci in the developing pallium during late neurogenesis.}

We previously reported that BAF complexes potentiate the activity of two main H3K27 demethylases, JMJD3 and UTX. Accordingly, elimination of BAF complexes during early corticogenesis leads to a global increase in repressive marks $(\mathrm{H} 3 \mathrm{~K} 27 \mathrm{Me} 2 / 3)$ and downregulation of gene expression at E13.5 (Narayanan et al., 2015b; Nguyen et al., 2016). In further analysis, we performed coimmunoprecipitation (CoIP) experiments on tissue lysates from the pallium of E17.5 wild-type (WT) embryos followed by mass spectrometry (MS) to identify BAF155/BAF170-interacting proteins. At E17.5, we found that BAF155 and BAF170 bind to the H3K27me2/3 demethylases, UTX/Kdm6a and JMJD3/Kdm6b, as shown in our previous study at E13.5 (Narayanan et al., 2015b). BAF155/BAF170 was also observed to interact with $\mathrm{H} 3 \mathrm{~K} 4 \mathrm{me} 1 / 2$ demethylase LSD1/Kdm1a in the E17.5 pallium (Figure 3.1A, B and Figure S3.1A).

To investigate if BAF complexes regulate epigenetic programs in late cortical development, we crossed BAF155-floxed (BAF155 fl/ff) mice and BAF170-floxed $\left(B A F 170^{f / f}\right)$ mice with the $h G F A P$-Cre line to generate dcKO mutants. In contrast to the Emx1-Cre line used in our previous studies (Tran et al., 2009; Tran et al., 2013; Narayanan et al., 2015b) with Cre recombination in the developing cortex as early as $\mathrm{E} 10.5$, the hGFAP promoter is not active in the pallium prior to E12.5. At E13.5, hGFAP-Cre activity is restricted to the medial pallium (MP), containing the hippocampal anlage and medial cortex (Figure S1B). From E15.5 onward, hGFAPCre activity extends to the dorsal pallium (DP; dorsal cortex) and lateral pallium (LP; lateral cortex) during development (Figure S3.1C). BAF155 and BAF170 proteins were not detected in the MP of dcKO mutants from E14.5, or in the entire VZ of the pallium from E15.5 onward (Figure S1D) (Narayanan et al., 2015b), hence validating our BAF155/BAF170 knock-out system in late pallial progenitors.

Given the identified interaction of BAF complexes with the H3K27me2/3 demethylases $\mathrm{Kdm6a} / \mathrm{b}$ and $\mathrm{H} 3 \mathrm{~K} 4 \mathrm{me1} / 2$ demethylase $\mathrm{Kdm} 1 \mathrm{a}$ in the E17.5 pallium, we next compared $\mathrm{H} 3 \mathrm{~K} 27 \mathrm{me} 3$ repressive and $\mathrm{H} 3 \mathrm{~K} 4 \mathrm{me} 2$ activatory marks in the $\mathrm{E} 17.5 \mathrm{dcKO}$ and control pallia. As reported previously, loss of BAF complexes in the E13.5 murine pallium in dcKO_Emx1-Cre mutants results in an increase in H3K27me3 levels (Narayanan et al., 2015b). 
A

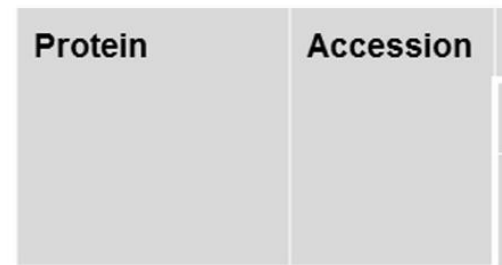

\begin{tabular}{l|l}
\hline Kdm6a/Utx & gil33859492
\end{tabular}

Kdm6b/JMJD3

Kdm1a/LSD1/Aof2

C

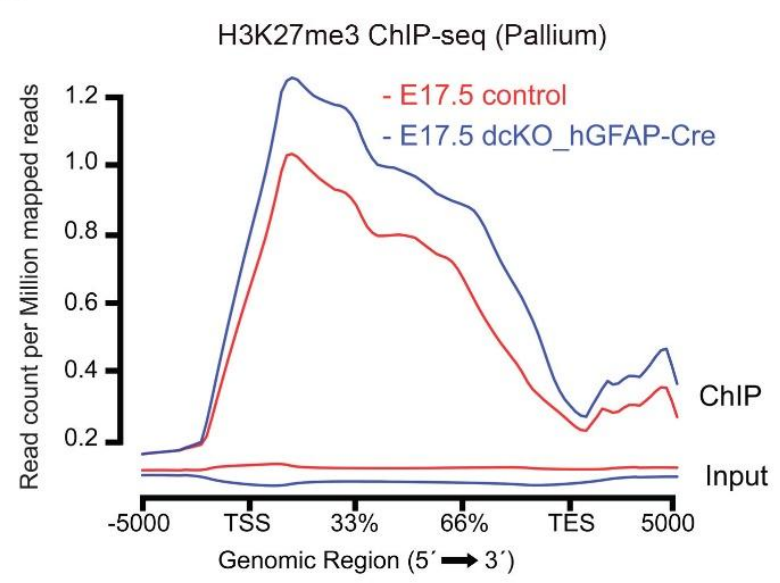

E

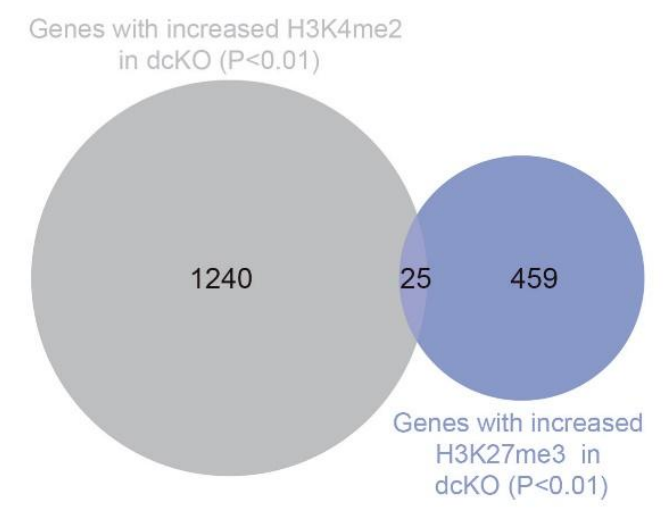

B

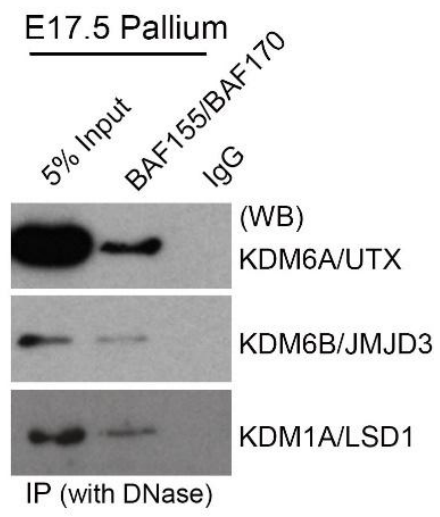

D

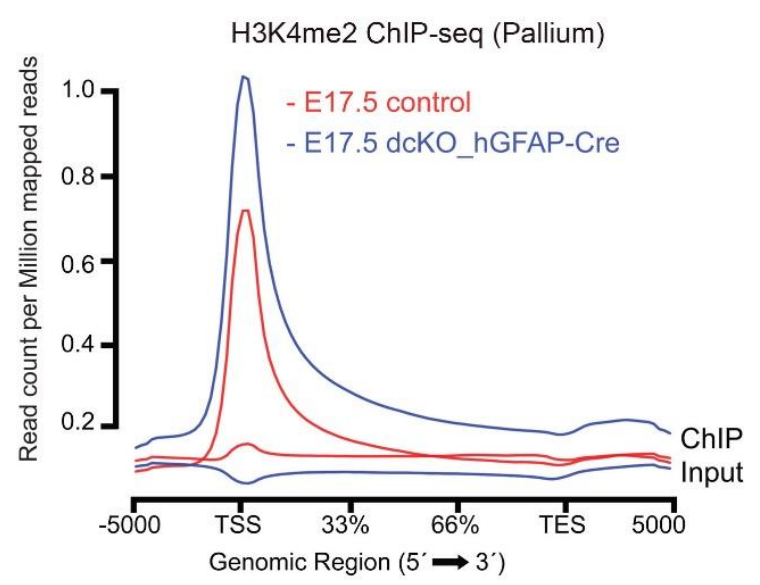

F

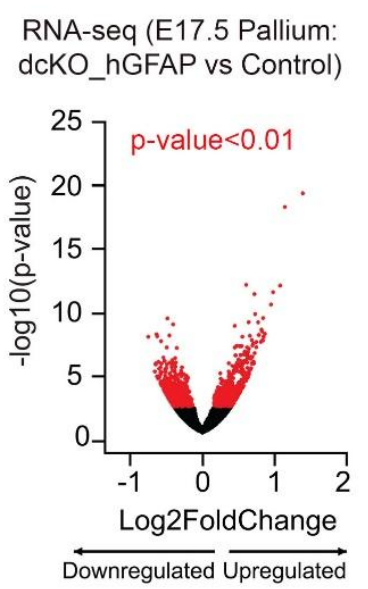


Figure 3.1. BAF complexes globally control epigenetic and gene expression programs in late development pallium. (A) Table showing the peptide number for Kdm6a, Kdm6b and Kdm1a proteins purified from BAF155, BAF170 immunoprecipitates of protein extracts from NS5 cells, E13.5 or E17.5 forebrain. (B) Interactions of BAF155 and BAF170 with Kdm6a, Kdm6b, and Kdm1a were confirmed by ColP/WB analyses of E17.5 pallium tissue. (C, D) Distribution of H3K27me3 (C) and H3K4me2 (D) marks along gene bodies in the dcKO and control pallium at E17.5. H3K27me3 levels are increased in dcKOs. dcKO (E) Genes with increased H3K4me2 or H3K27me3 marks in the dcKO pallium at E17.5 are largely non-overlapping. (F) Volcano plot representing differentially regulated genes in the dcKO pallium at E17.5.

Similarly, ChIP-seq analysis performed using chromatin isolated from the E17.5 dcKO pallium also revealed an increase in H3K27me3 upon BAF155/170 knock-out (Figure 1C). Specifically, 181 genes showed a significant increase in these marks around their transcription start site (TSS) regions ( $\pm 2000 \mathrm{bp}$ ) compared to 13 genes that showed a decrease, a difference that likely reflects secondary effects and/or compensatory mechanisms. H3K27me3 is a broad chromatin mark localized not only at TSS but also spread over gene bodies. We also looked at the number of genes with altered H3K27me3 at their coding regions (including TSS). There were 484 genes with increased and 156 genes with decreased H3K27me3 (Figure 3.1E). Strikingly, loss of BAF complexes in late corticogenesis resulted in a concurrent increase in activatory H3K4me2 marks in the E17.5 pallium (Figure 3.1D), with 1265 genes showing a significant increase in this mark around their TSS (Figure 3.1E). Only 112 genes showed decreased H3K4me2, which again may represent some secondary effects. Importantly, genes affected by increased H3K27me3 and H3K4me2 were largely distinct (Figure 3.1E).

We also performed gene expression profiling of the dcKO pallium at E17.5 (Figure 1F). In contrast to the globally reduced gene expression in the dcKO_Emx1-Cre pallium at E12.5 (Narayanan et al., 2015b), at E17.5, we found nearly equal number of downregulated and upregulated genes in the dcKO pallium (Figure 3.1F). 
Collectively, these data indicate that loss of BAF complexes during late corticogenesis induces an increase in activatory $\mathrm{H} 3 \mathrm{~K} 4 \mathrm{me} 2$ and repressive H3K27me3 marks at distinct sets of genes, thereby pointing to possible dual functions of BAF complexes as both activators and repressors in late cortical neurogenesis.

\subsubsection{Conditional inactivation of BAF complexes during late cortical development impairs neurogenesis of upper cortical layer neurons and the hippocampus.}

We selected the downregulated genes in the E17.5 dcKO pallium in RNA-Seq and subjected them to functional category analysis. They are enriched in neuronal differentiation-related categories (Figure 3.2A), and showed an overall increase in H3K27me3 mark (Figure 2B). Most of the differentiation-related genes that were significantly downregulated in dcKO mice (Table S3) showed an increase in H3K27me3. For some selected candidates, we also confirmed their downregulation and increased H3K27me3 by qPCR and ChIP-qPCR respectively (Figure S3.2A, B).

Next, we asked if these genes with decreased expression and increased H3K27me3 are directly bound by the BAF complexes. We made use of a previously published ChIP-Seq dataset (GSE37151) for Brg1 in the developing mouse forebrain (Attanasio et al., 2014). Strikingly, the majority of genes that showed increased H3K27me3 in dcKO cortices were also bound by Brg1 (Figure S3.2C), with sites of increased H3K27me3 co-localizing with Brg1 binding sites (Figure 3.2D). 
A Neuronal differentiation-related pathways are downregulated in Mutants: $\square$ dcKO_hGFAP-Cre vs Control (RNA-seq)

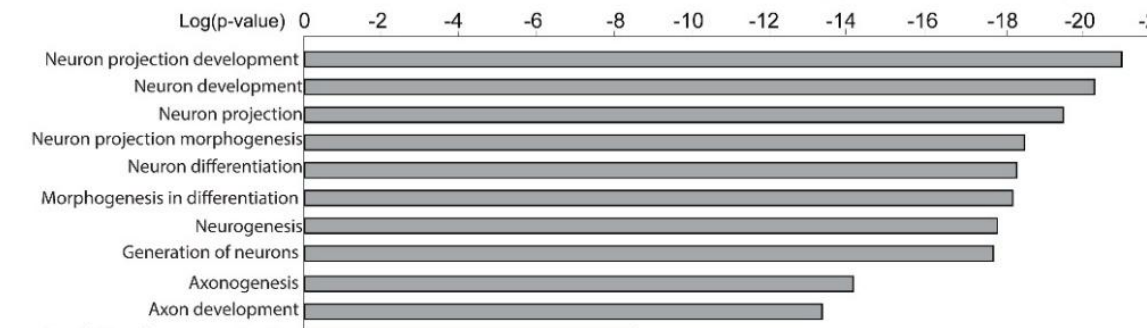

Regulation of neuron projection

Regulation of neuron differentiation

Regulation of neurogen

Dendrite development

Regulation in differentiation

Regulation of dendrite development

Regulation

$$
\text { Neuron maturation }
$$

B Regulation of axon extension

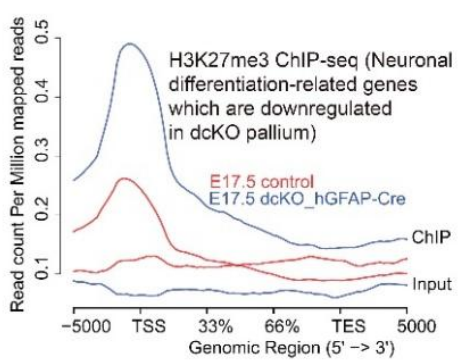

=Control =dcKO hGFAP-Cre - Exon - Intron

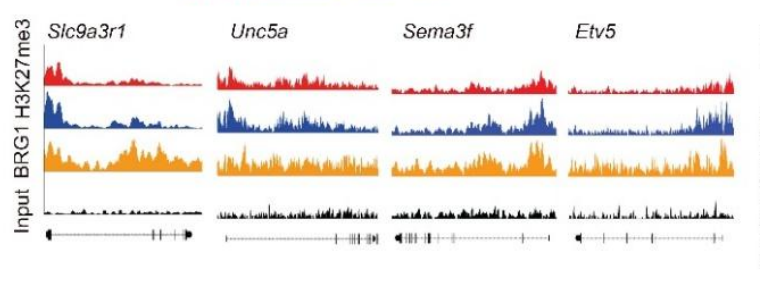

E Control

dcKO_hGFAP-Cre

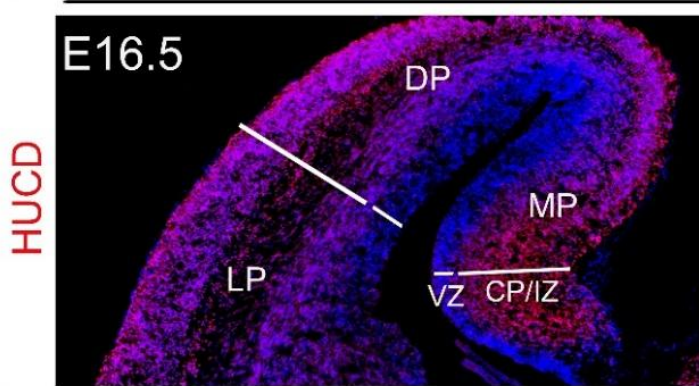

$\mathbf{F}$
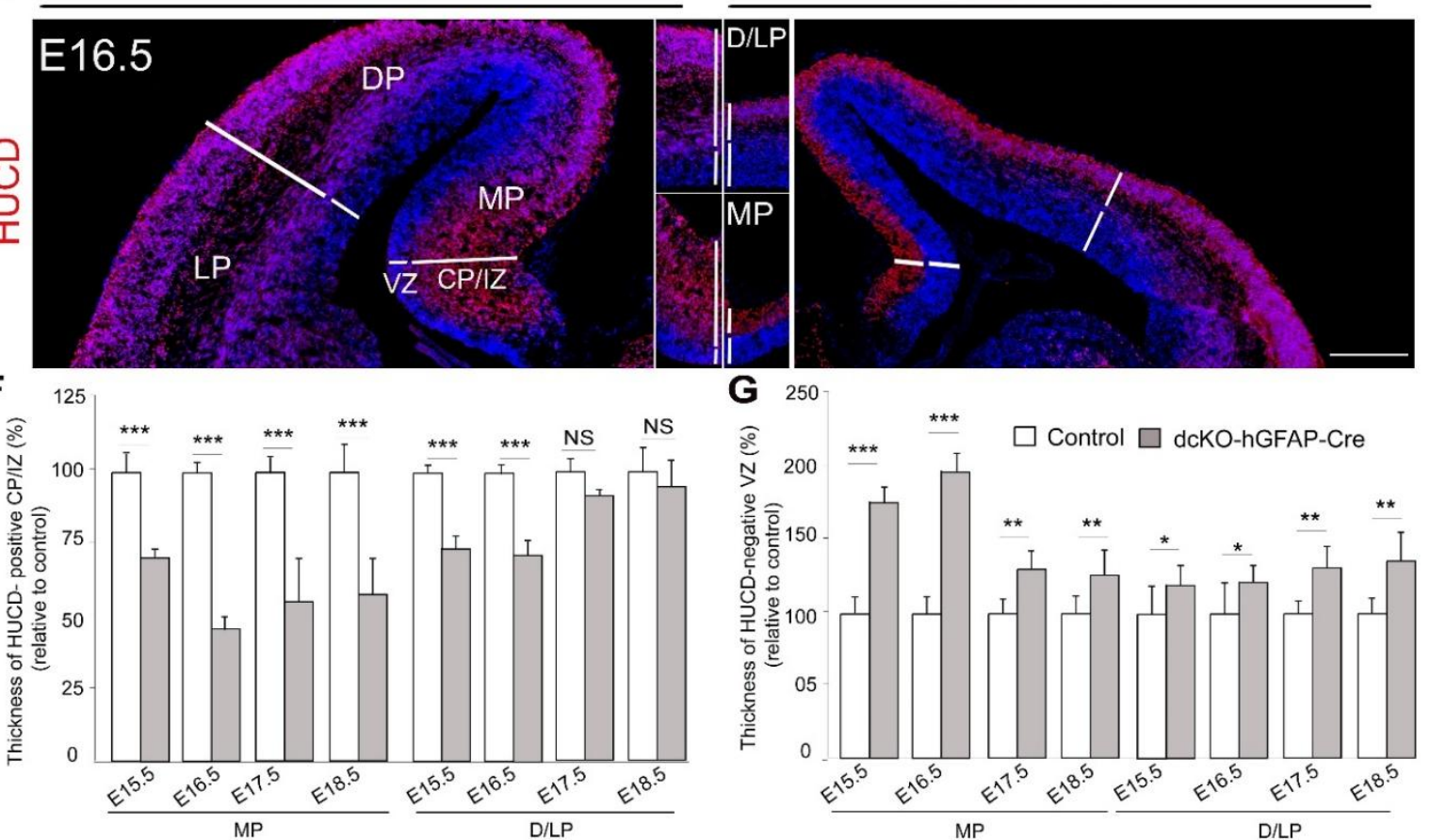

G 250

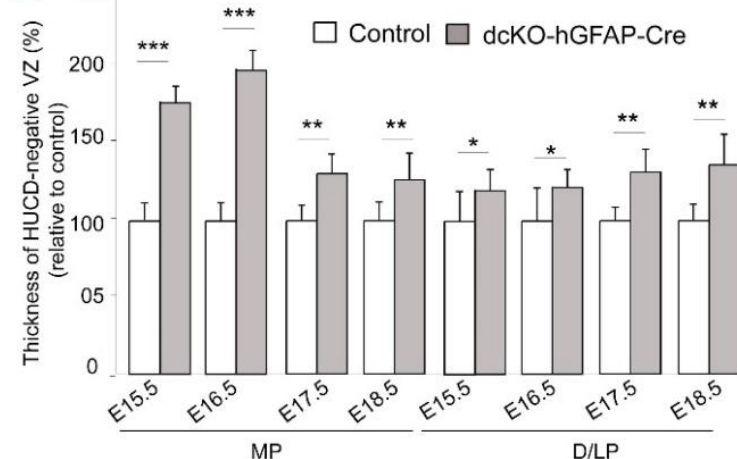


Figure 3.2. H3K27me3-linked silencing of neuronal differentiation-related genes in BAF complex-deleted pallium in late stages.

(A) Neuronal differentiation-related genes are downregulated in the dcKO pallium at E17.5.

(B) General H3K27me3 profile plot of neuronal differentiation-related genes that are downregulated in dcKO pallium. (C) Upper panel: Heatmap depicting the changes in H3K27me3 levels at neural differentiation-related genes that are downregulated in dcKO pallium at E17.5 individually. Lower panel: Average relative $\mathrm{H} 3 \mathrm{~K} 27 \mathrm{me} 3$ binding levels on those genes combined. (D) Integrated genome browser views of H3K27me3 and Brg1 (GSE37151 (Attanasio et al., 2014)) binding along representative neural differentiation-related genes downregulated in dcKO pallium. $(E-G)$ IF $(E)$ and quantitative $(F, G)$ analyses indicate that the loss of BAF155 and BAF170 leads to a diminished thickness of the HuCD+ ${ }^{+}$cortical plate (CP) and intermediate zone (IZ) $(F)$, and expanded thickness of the HuCD ${ }^{-} V Z(G)$ in the entire pallium at E15.5-E18.5. Values are presented as means \pm SEMs $\left(n>3,{ }^{\star} P<0.05\right.$, $\left.{ }^{* \star} P<0.01,{ }^{* \star *} P<0.005\right)$. Abbreviations: VZ, ventricular zone; $\mathrm{CP}$, cortical plate; IZ, intermediate zone; MP, medial pallium; DP, dorsal pallium; LP, lateral pallium. Scale bar $=100 \mu \mathrm{m}(\mathrm{E})$

We further confirmed these observations in a reverse approach, in which we first selected the genes with increased H3K27me3 in E17.5 dcKO (Figure S2D) and subjected them to functional category analysis. Again, they also mostly fell under neuronal differentiation-related categories (Figure S3.2E). We then examined their expression in our RNA-Seq analysis. As expected, most of them were downregulated in dcKO embryos.

Because the hGFAP promoter is active early in the MP (from E13.5) and later in the DP and LP (from E14.5) (Figure S3.1B, C), we compared neuronal differentiation between controls and dcKO mutants, in both the MP and at the area between the DP and LP (D/LP). Neurogenesis in late (E15.5-E17.5) development of the pallium in dcKO mutants was decreased, as evidenced by a decrease in the thickness of the cortical plate (CP) and intermediate zone (IZ), marked by the expression of the panneuronal markers HuCD, TUBB3 and NeuN in both the cortex (D/LP) and hippocampus (MP) (Figure 3.2E, F). Consistent with this, immunofluorescence (IF) analyses of neuronal subtype markers indicated that loss of BAF155 and BAF170 led to a significant decrease in the number of late-born Satb2 ${ }^{+}$or $\mathrm{Brn}^{+}$neurons, but not early-born Tbr1+ neurons, in the DP and LP (Figure S3.3A-D). 
To study neurogenesis specifically in the MP, we performed IF on sections from E15.5-E17.5 control and dcKO embryonic brains using the antibody Zbtb20 (Figure S3.3E), which outlines the hippocampal anlage as early as E14.5 and is confined postnatally to hippocampal cornu ammonis (CA1-CA3) regions. Zbtb20 staining revealed remnants of the hippocampus proper (Figure $3.3 \mathrm{C}$ ) in mutants compared to controls at all examined stages, E14.5-E17.5 (Figure S3.3E, G). Indeed, three-dimensional (3D) reconstruction of Zbtb20 expression also revealed a substantial reduction in the volume of the developing hippocampus in dcKO embryos at E15.5 (Figure S3.3H). Consistently, immunostaining of the dentate gyrus (DG) with its specific marker Prox1, indicated agenesis of this hippocampal domain (Figure S3.3F, I). In the DP/LP of mutants, whereas the generation of lower layer (Tbr1+/L6, and CTIP2 $+/ L 5)$ neurons was only mildly decreased, the number of late-born Satb2 ${ }^{+}$, and Brn2 ${ }^{+}$L4-L2 neurons was strongly diminished (Figure 3.3A, B). In further support, we found that BAF complexes control expression of sets of gene exerting important roles in generation of cortical layers and hippocampal development. 
Chapter 3

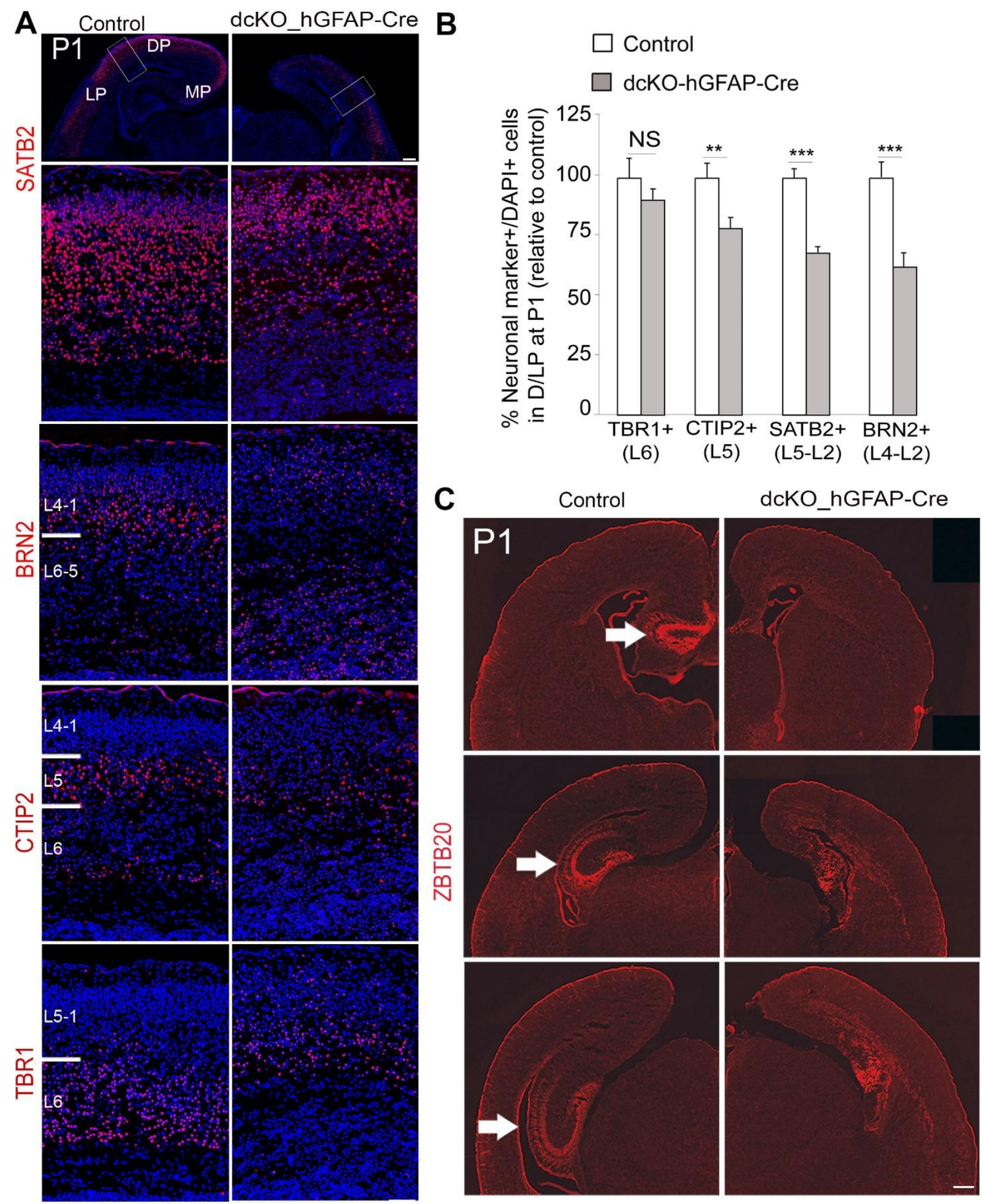


Figure 3.3. BAF complexes are required for the formation of cortical upper layers and the hippocampus.

(A-B) IF (A) and statistical (B) analyses of cortical phenotypes at P1 in a comparable dorsal/lateral area, immunostained for the indicated neuronal layer markers. (C) IF analysis of Ztbt20 revealed that the hippocampus is underdeveloped in mutants (denoted by arrow). Values are presented as means \pm SEMs $\left(n>3,{ }^{* *} P<0.01,{ }^{* *} P<0.005\right)$. Abbreviations: MP, medial pallium; DP, dorsal pallium; LP, lateral pallium: L: layer. Scale bars $=100 \mu \mathrm{m}(A)$, $50 \mu \mathrm{m}(\mathrm{B})$, and $100 \mu \mathrm{m}(\mathrm{C})$.

To gain additional evidence about how the defect in neuronal differentiation is caused by increased level of H3K27me3, we used GSK-J4, a potent selective H3K27 demethylase (JMJD3 and UTX) inhibitor (Kruidenier et al., 2012). The elevated level of H3K27me3 by GSK-J4 administration significantly decreased the number of late-born Satb2 ${ }^{+}$and Cux1+ neurons (Figure S3.4A-D), as observed in dcKO pallium with enhanced level of H3K27me3.

Together, these findings suggest that deletion of BAF complexes in late NSCs leads to H3K27me3-linked silencing of neuronal-differentiation genes and results in diminished late cortical and hippocampal neurogenesis.

\subsubsection{The NSC pool is increased at late development stages in the dcKO pallium.}

Our previous data indicated that the loss of BAF complexes leads to large-scale downregulation of gene expression in early cortical development (Narayanan et al., 2015b; Nguyen et al., 2016). Intriguingly, the late elimination of BAF complex function also led to upregulation of a substantial number of genes. In order to assess the role of the genes upregulated in dcKO embryos, we applied the aforementioned strategy. Functionally, they mainly converged into cell proliferation-related categories (Figure 3.4A). Moreover, these genes also showed an overall increase in $\mathrm{H} 3 \mathrm{~K} 4 \mathrm{me} 2$ in the dcKO pallium (Figure 3.4B). 
Next, we assessed the changes in H3K4me2 levels at their individual TSS regions. As expected, most of them had an increase in this activatory mark with the overall trend being highly significant (Figure 3.4C) and they mostly converged into cell cycle-related groups (Figure S3.2D, S2E). The sites of increased H3K4me2 also substantially overlapped with BAF complex (Brg1) binding (Figure 3.4D, Figure S3.2C). Selected candidates were confirmed by qPCR and ChIP-qPCR (Figure S2A, S2B). Because the expression of genes encoding $\mathrm{H} 3$ demethylases LSD1/kdm1a, UTX/Kdm6a, JMJD3/Kdm6b was unaltered in dcKO cortex in our RNA-seq experiment, it is possible that BAF complexes control the methylation of H3K4 and H3K27 through mechanisms other than activating or inhibiting the expression of genes coding for these H3 demethylases. Our earlier study indicated that BAF complexes potentiate the H3K27 demethylase activity of UTX/Kdm6a and JMJD3/Kdm6b (Narayanan et al., 2015b), which encouraged us to investigate whether endogenous BAF155 and BAF170 are required for full H3K4 demethylase activity of LSD1/kdm1a. We therefore performed the histone demethylase KDM1/LSD1 activity quantification assay (see Material and Methods). The results revealed that significantly less H3K4 is demethylated in BAF155/BAF170-ablated NSCs compared to control counterparts (Figure S3.2G). 
A
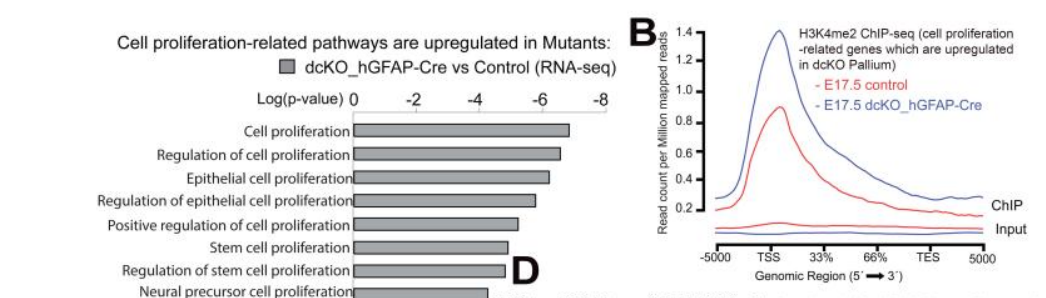

C

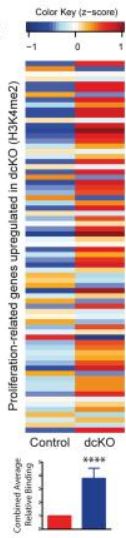

E
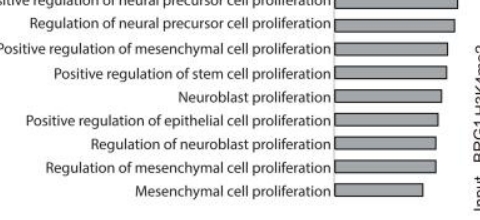

Adk

Cond1

Ctnnb1

Pitx2

ak. Lin i. I.. I... L..

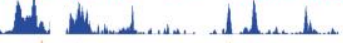
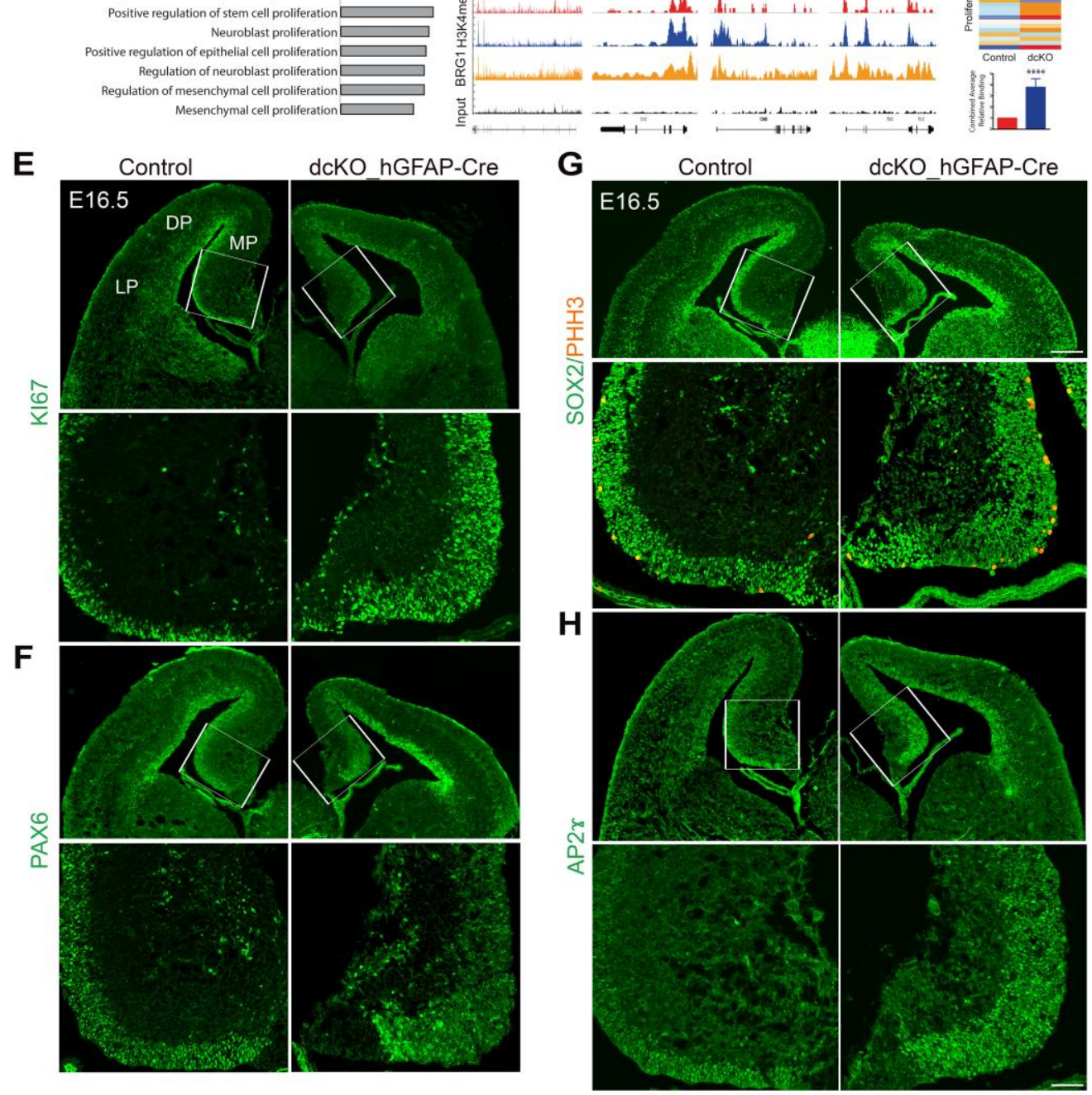

I

J

$\square$ Control $\square$ dcKO-hGFAP-Cre

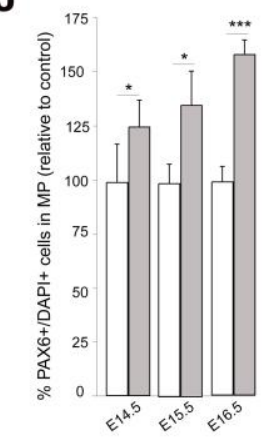

K

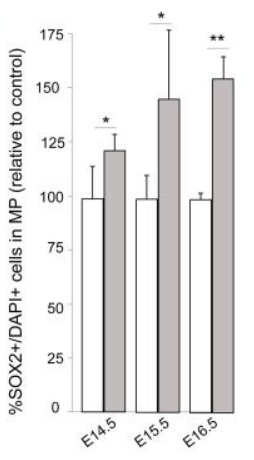

$\mathbf{L}$
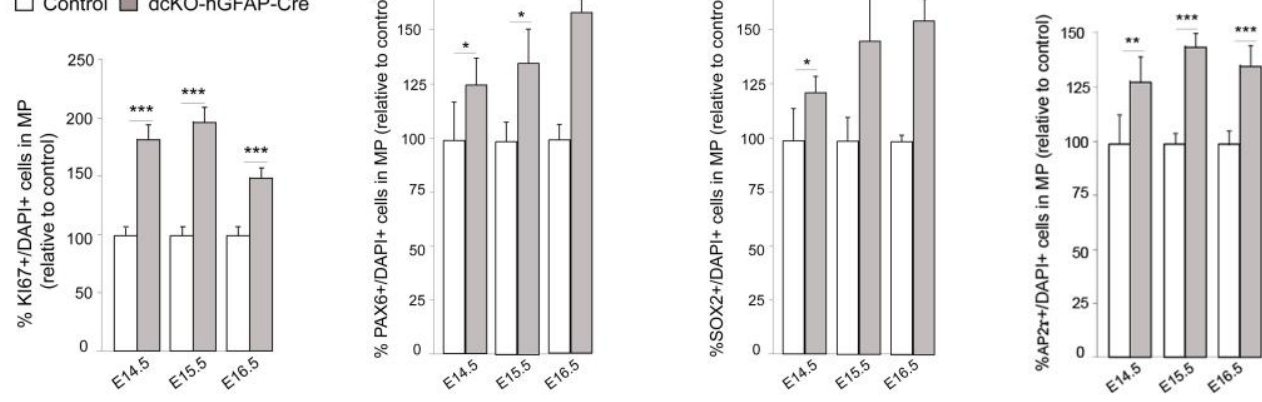
Figure 3.4. Loss of BAF155 and BAF170 causes H3K4me2-linked upregulation of genes involved in the mitotic cell cycle and proliferation in late cortical development.

(A) Proliferation-, cell-cycle-related genes are upregulated in the dcKO pallium at E17.5.

(B) General H3K4me2 profile plot at proliferation-related genes that are upregulated in dcKO pallium. (C) Upper panel: Heatmap depicting the changes in H3K4me2 levels at proliferation-related genes that are upregulated in dcKO pallium at E17.5. Lower panel: Average relative $\mathrm{H} 3 \mathrm{~K} 4 \mathrm{me} 2$ binding levels on those genes combined. (D) Integrated genome browser views of H3K4me2 and Brg1 binding (GSE37151) (Attanasio et al., 2014) along representative proliferation-related genes upregulated in dcKO pallium. $(\mathrm{E}-\mathrm{H})$ Representative images showing IF analyses of coronal sections of control and dcKO pallium at E16.5 using antibodies that specifically label the indicated NSC markers. Lower panels: Higher-magnification images of areas indicated by white boxes. (I-L) Quantitative analyses indicated increased numbers of NSCs in the MP of dcKO mutants at the indicated stages. Values are presented as means \pm SEMs $\left(\mathrm{n}>3,{ }^{\star} P<0.05,{ }^{* \star *} P<0.01,{ }^{\star \star *} P<0.005\right)$. Abbreviations: TSS, transcription start site; TES, transcription end site; MP, medial pallium; DP, dorsal pallium; LP, lateral pallium. Scale bars: $100 \mu \mathrm{m}(\mathrm{G})$ and $50 \mu \mathrm{m}(\mathrm{H})$.

IF analysis of the expression of HuCD, TUBB3, NeuN indicated an enlargement of the VZ in the dcKO pallium, more strongly in MP than in D/LP (Figure 3.2E, G). Reconstruction analyses showed that the volume of the hippocampal neuroepithelium, as revealed by Pax6 expression, is larger in the mutant MP (Figure S3.5A, B). These data suggest increased pools of progenitors in proliferative zones of the dcKO pallium. Indeed, more $\mathrm{Ki} 67^{+}$mitotically active cells were found in mutants than in controls (Figure 3.4E, I; and Figure S3.5C). We then examined pools of RGs and intermediate progenitors (IPS) (Figure 3.4F-H, 3.4J-L and Figure S3.5D-G). Similar to the increased number of $\mathrm{Ki}_{67}{ }^{+}$mitotic cells, the number of $\mathrm{Pax}^{+}, \mathrm{Sox}^{+}$, and AP $2 \gamma^{+}$NSCs in the VZ gradually increased from E14.5 in the mutant MP (Figure 3.4F-H). Notably, the effect was more profound in the NSC pool in the MP than in the D/LP (Figure S3.5C-F). This possibly relates to the spatiotemporal hGFAP-Cre activity, exerting early activity in the MP (Figure S3.1B, C). In contrast to the increased number of RGs, the number of Tbr2+ IPs was decreased (Figure S3.5D, G), indicating a failure of neuronal differentiation in the mutant pallium. 
To substantiate the effect of the H3K4me2 level on the cortical NSC pool, we examined an increased H3K4me2 by using ( \pm )-trans-2-Phenylcyclopropylamine hydrochloride (2-PCPA), a specific inhibitor of LSD1 histone demethylase. 2-PCPA has been shown to increase H3K4me2 in mouse brain (Sun et al., 2010). The treatment of 2-PCPA also led to an increased pool of $\mathrm{Pax6}^{+}$, Sox2 ${ }^{+} \mathrm{NSCs}$ in developing cortex (Figure S3.4E-G).

Together, these findings suggest that, in the absence of BAF155/BAF170, at late corticogenesis (E14.5-E17.5), NSCs in the VZ are kept in the proliferative phase rather than differentiating into IPs and/or neurons.

\subsubsection{RGs acquire a NE-like identity in the BAF155/BAF170-deficient pallium.}

The appearance of RGs in the pallium is marked by initiation of the expression of the astrocytic differentiation markers, GLAST and BLBP at E12.5 (Hartfuss et al., 2001; Sahara \& O'Leary, 2009). At E13.5 and E14.5, expression level of GLAST and BLBP is comparable between control and mutants (Figure S3.5H-L). Following IF analysis at later stages (E15.5-E16.5) we observed that despite the increased number of Pax6 $/$ Sox2+/AP2 $\gamma^{+}$NSCs (Figure 4F, G), immunopositive signals for GLAST and BLBP were diminished in the DP/LP and largely undetectable in the MP in dcKO mutants (Figure 3.5A, D).

Another hallmark for NE-RG cells transition is the replacement of tight junctional complexes (NE trait) with adherens junctions (RGs trait) (Aaku-Saraste et al., 1996; Sahara \& O'Leary, 2009). Notably, we found that during late corticogenesis, many genes encoding for tight junction proteins (such as AMOT, MPP5, Occludin, INADL, PKD2, DLG1, CFTR, TGFB3) were significantly upregulated and also those involved in adherens-junction proteins (e.g. TNS3, PLEC, PTK2B, ZO1, a-Catenin, KIAA1462/Jcad, filamin, CAMSAP3, APC, MYH9, MYO1E, SPTAN1, ITGA1, RND1) were downregulated in the dcKO pallium. Additionally, we examined VZ expression of Occludin (a tight junction marker) and ZO1, $\alpha$-Catenin (adherens-junction markers) localized at the apical surface. Occludin is normally downregulated in NEs as they 
differentiate into RGs (Aaku-Saraste et al., 1996; Sahara \& O'Leary, 2009). At E13.5-E16.5, expression of occludin at the apical surface of the VZ in the control pallium was undetectable, whereas its expression was strongly upregulated in the dcKO pallium at E15.5-E16.5 (Figure 3.5C, D; Figure S3.5I). The expression of adherens junction markers ZO1, and $\alpha$-Catenin at the apical surface of RGs was not affected at E13.5-E14.5 (Figure S3.5J-L), but largely absent at E16.5-E17.5 in dcKO cortex (Figure 3.5B, D).

BAF complexes seem not only to block NE fate in late pallium development, but also control the differentiation from NEs to RGs, as evidenced by downregulated expression of the RG markers BLBP and GLAST together with upregulated expression of the NE marker Occludin in the dcKO_Emx1-Cre cortex at E13.5 (Figure S3.5M-O). Thus, our data revealed that the downregulation of the expression of astroglial and adherens-junction markers is correlated with upregulation of tight-junction markers in late cortical development of dcKO mutants. These complementary datasets indicate that deletion of BAF complexes during late development of the pallium dedifferentiates RGs to NE-like cells. 
Chapter 3

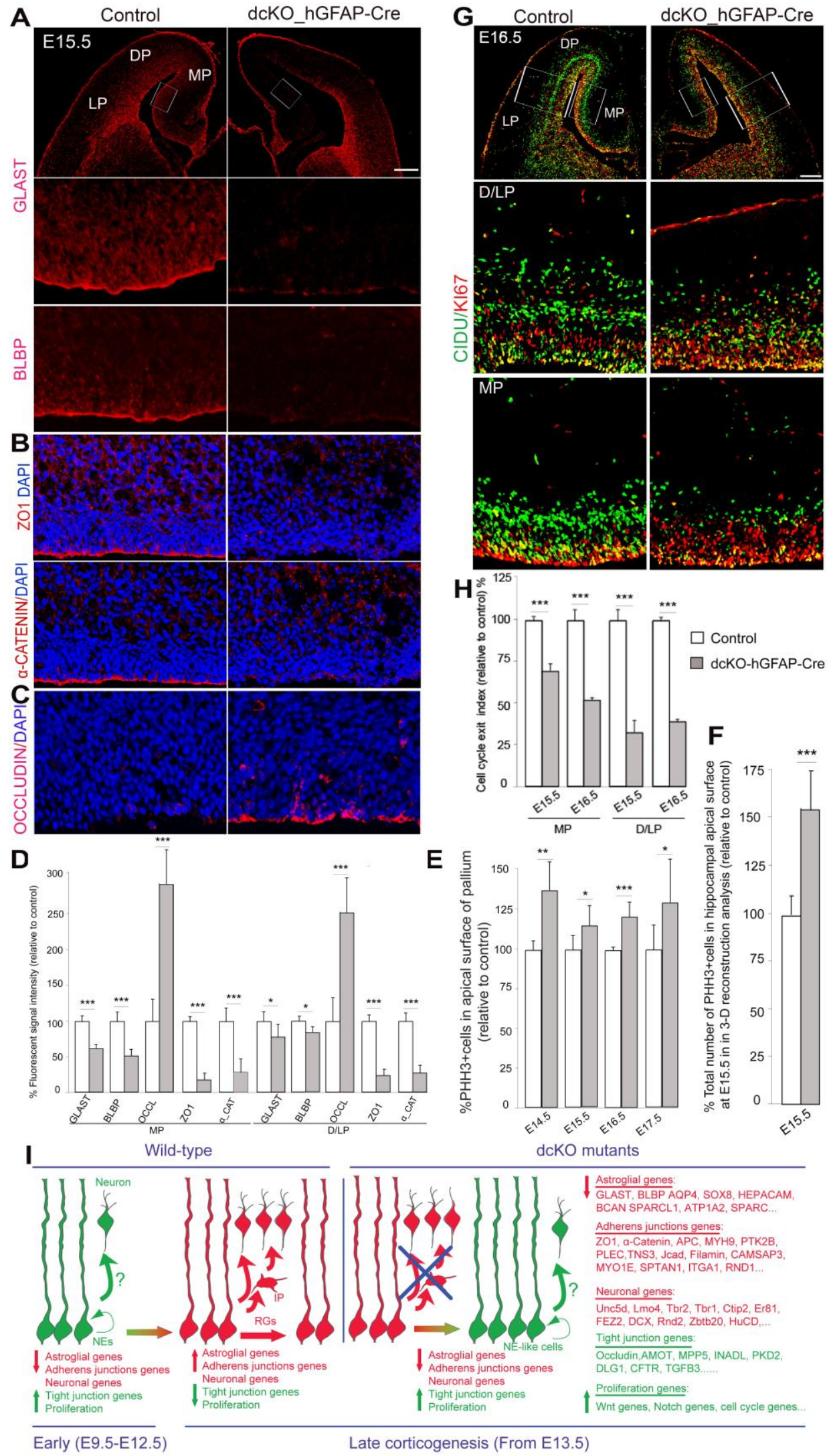


Figure 3.5. NE-like cells in the BAF-complex-deleted pallium in late development retain their highly proliferative competence.

(A-C) Immunostaining of the control and dcKO pallium sections at E15.5 for indicated markers

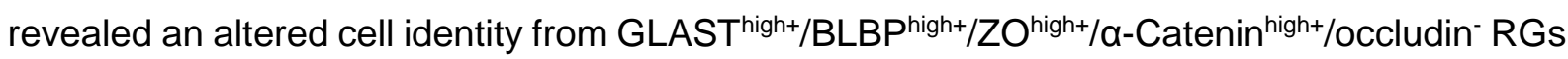
in controls to GLAST ${ }^{\text {low+}} / \mathrm{BLBP}^{\mathrm{low}+} / \mathrm{ZO}^{\mathrm{low}+} / \alpha_{-}-$Catenin $^{\text {low+ }} /$ occludin $^{\text {hight }}$ NEs in dcKO MP. (D) Quantification and statistical analysis of panels $A-C$ are shown. (E-F) Quantitative analyses showing that the loss of BAF155 and BAF170 leads to an increase in mitotic $\mathrm{pHH}^{+}$ RGs in the pallium at E14.5-E16.5 (E). Note that quantification of $\mathrm{pHH} 3^{+}$cells $(\mathrm{F})$ was done in the entire developing hippocampus $\left(\mathrm{Pax}^{+} / \mathrm{Ztbt}^{2} \mathrm{O}^{+}\right)$at $\mathrm{E} 15.5$ using $3 \mathrm{D}$ reconstruction (see also Figure S5A-B, Movie S1). (G) Images showing double IF at E16.5 for CldU, and Ki67 in control and dcKO mutants. $(\mathrm{H})$ Quantitative analyses showing a significantly lower exit index (number of $\mathrm{CidU}^{+} / \mathrm{Ki}^{-} 7^{-}$cells per total number of $\mathrm{CidU}^{+}$cells) in mutants in D/LP and MP areas than in controls. (I) Schema illustrating that a higher proportion of RG progenitors in the late-stage (from E13.5) dcKO pallium acquire NE-like identity (i.e. downregulated expression of astroglial, adherens-junction, differetiation genes and upregulated expression of tight-junction, proliferation genes). Values are presented as means \pm SEMs $\left(n>3,{ }^{\star} P<0.05\right.$, $\left.{ }^{* * *} P<0.01,{ }^{* *} P<0.005\right)$. Abbreviations: NE, neuroepithelial cell; RG, ventricular radial glial progenitors, Hi, hippocampus; Cx, cortex; MP, medial pallium; DP, dorsal pallium; LP, lateral pallium. Scale bars $=100 \mu \mathrm{m}(\mathrm{A}, \mathrm{G})$.

\subsubsection{Change in spindle orientation, and increased proliferative capacity of NSCs in the BAF155/BAF170-deficient pallium.}

To assess the implications of the retention of a NE-like identity in the mutant cortex, we first found out if the dedifferentiation from RGs to NE-like cells was consequent to/caused altered spindles orientation. We stained E15.5-E16.5 sections from control and dcKO pallium using antibodies against pVim and pHH3 to mark mitotic cells and chromatin, respectively (Figure S3.5A). The division angles of apical RGs were quantified and categorized based on cleavage angle: vertical (60-90\%), oblique $\left(30-60^{\circ}\right)$, and horizontal $\left(0-30^{\circ}\right)$. Notably, more progenitors with vertical cleavage were detected in the mutant pallium than controls (Figure S3.5A, B), suggesting that the loss of BAF complexes in late pallial development induces proliferative symmetric divisions, which mainly generate NEs and RGs. 
The increased number of $\mathrm{Pax6}^{+} / \mathrm{Sox} 2^{+} \mathrm{NSC}$ suggested that RGs were kept in the cell cycle to promote their proliferation, instead of exiting to become neurons. To ascertain whether loss of functional BAF155 and BAF170 leads to altered cell proliferation, we labeled M-phase cells by immunostaining with an anti-pHH3 antibody. Quantitative comparisons of immunostained medial brain sections of the E14.5-E17.5 pallium (Figure 3.4A, 3.5D) and 3D reconstruction analyses of the entire hippocampus at E15.5 (Figure 3.5E), indicated that the loss of BAF155 and BAF170 resulted in an increased number of $\mathrm{pHH}^{+}$cells in the pallium (Figure 3.5D, E).

To better characterize BAF155/BAF170 loss-of-function effects on neuronal differentiation, we next used a thymidine analog (CldU) injection paradigm (24-h CldU pulse-labeling) to establish a quantitative in vivo cell cycle exit index in the developing MP and D/LP (Figure 3.5G, H). We also performed double-labeling IF using antibodies against CldU to label both cycling progenitors and those that recently exited the cell cycle, and Ki67 for proliferating progenitors in all cell cycle phases. Statistical analyses revealed a significantly lower cell cycle exit index in dcKO mutants compared to controls (Figure 3.5G, H). To examine whether BAF155/BAF170-deficient NSCs undergo several proliferative rounds, we again detected the sequential incorporation of different thymidine analogues (CldU, IdU) into cortical NSCs (Figure S3.6C). Given that cell cycle length of cortical progenitors between E14.5-E16.5 is about 15h-18h per cycle (Takahashi et al., 1995), pregnant mice were injected with CldU (at E14.5) and IdU (at E15.5). Tissue was collected at E16.5 and processed for IHC analysis with antibodies against CldU, IdU and Ki67 (Figure S3.6D). The cortical cells between 1-3 successive rounds of cell division were labeled as following: (1) Cells from the first and the second cell divisions as well as their progenies are marked with CldU (in green) and with IdU (in violet) respectively; (2) cells in third mitotic cell cycle will be labeled by Ki67 (red). Our statistical analysis (Figure S3.6E) indicated that between E14.5-E16.5, many cortical progenitors exit from the first ( $\mathrm{CldU}^{+} / \mathrm{ldU} / \mathrm{Ki67} 7^{-}: 6 \pm 0.83 \%$ in control and $0.7 \pm 0.38 \%$ in dcKO, green part of chart) and second $\left(\mathrm{CldU}^{+} / \mathrm{ldU}^{+} / \mathrm{Ki}^{-} 7^{-}: 73 \pm 2.52 \%\right.$ in control and $31.82 \pm 3.30 \%$ in dcKO, violet part of chart) cell cycles in control cortex, whereas a large fraction of NSCs further enters third ( $\mathrm{CldU}^{+} / / \mathrm{dU}^{+} / \mathrm{Ki} 7^{+}: 20.16 \pm 1.94 \%$ in control and $67.47 \pm 9.70 \%$ in $\mathrm{dcKO}$, red part of chart) cell cycle in dcKO cortex. 
We also examined apoptosis at different stages from E14.5 to E18.5 in the MP by performing IF for Casp3 (Figure S3.6F-K). Compared to controls, significantly higher numbers of dying cells were found in the MP of dcKO pallium (Figure S6F-J). Particularly, most apoptotic cells in mutants were Pax6 ${ }^{+}$RGs, while apoptotic Tbr2+ IPs were detected at a lesser extent. Apoptotic $\mathrm{HuCD}^{+}$neurons were rarely detected (Figure S3.6F-I, K), and this paralleled our previously observed apoptotic effect of selective loss of BAF complex in post-mitotic neurons The latter effect is further supported by the observation that selective elimination of BAF155 and BAF170 in post-mitotic neurons in dcKO_Nex-Cre had no effect on the populations of Ctip2+/Ztb20+ neurons, Pax6+/Sox2+ NSCs (Figure S3.6L-N) or Casp3+ apoptotic cells (Narayanan et al., 2015b).

Collectively, these findings indicate that the deletion of BAF complexes results in H3K4me2-linked activation of proliferation- and cell-cycle-associated genes. This resulted in three main morphogenetic defects of the dcKO pallium: (1) an expanded pool of NSCs, (2) diminished neurogenesis in late corticogenesis, and (3) malformed late-formed structures such as upper cortical layers and the hippocampus.

\subsubsection{Elimination of BAF155 and BAF170 de-represses Wnt signaling in late corticogenesis.}

Previous work has suggested that enhanced Wnt signaling promotes cortical NSCs proliferation (Chenn \& Walsh, 2002; Machon et al., 2007). We found that, during late corticogenesis, a considerable number of genes involved in Wnt signaling, including many Wnt target genes, were significantly upregulated in the dcKO pallium (Figure 3.6A, B; Figure S3.7A). These genes showed an overall (Figure 3.6C, D) increase in H3K4me2 levels. Moreover, their TSS regions, where increased H3K4me2 is observed in dcKO embryos, also coincide with Brg1 binding sites (Figure 3.6E). 
A

Wnt-related pathways are upregulated in Mutants: $\square$ dcKO_hGFAP-Cre vs Control (RNA-seq)

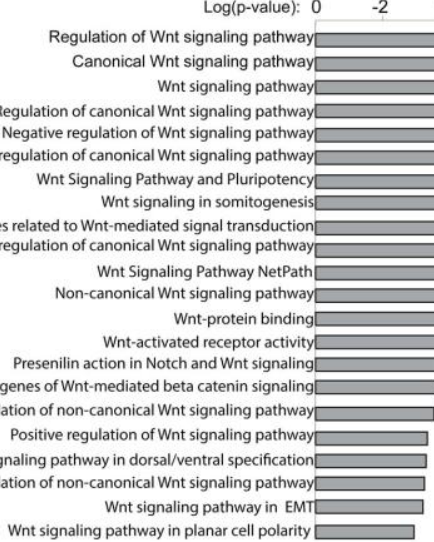

B

$\square$ dcKO_hGFAP-Cre vs Control (RNA-seq)
C

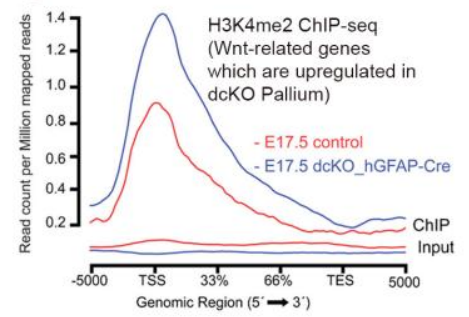

H3K4me2 ChIP-seq (E17.5 Pallium)

-Control $=$ dcKO hGFAP-Cre - Exon Intron

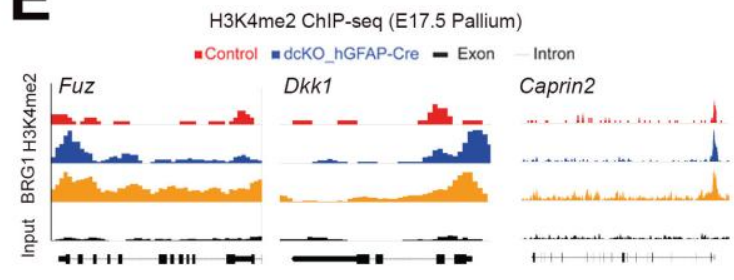

$1.0 \quad P$-value $<0.01$. Fold change $>1.2$
Tnfrsf11 Sfrp1 Dkk1

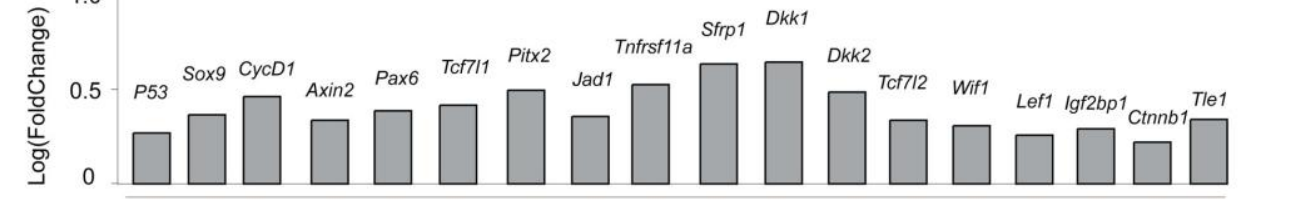

$\mathbf{F}$

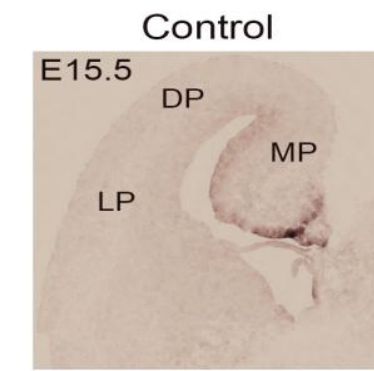

E16.5
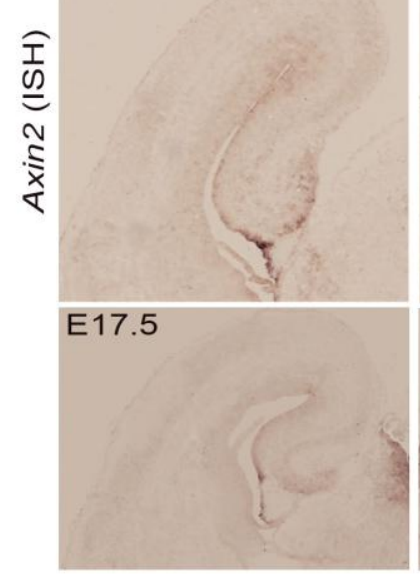

Wnt Targets dcKO_hGFAP-Cre
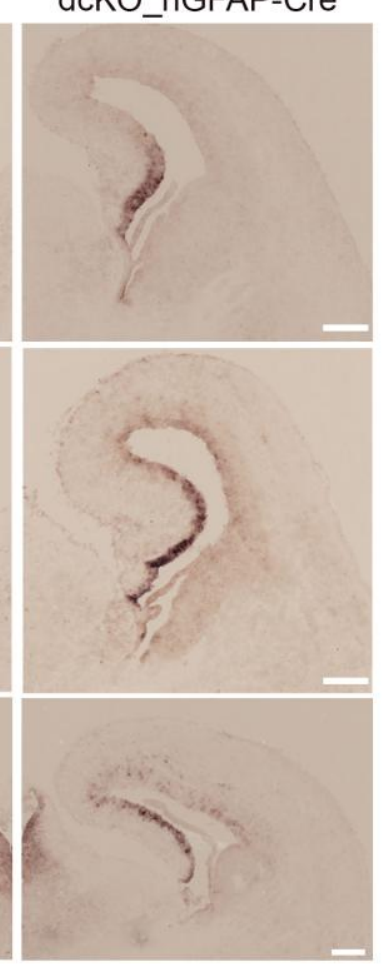

\section{G}

$\mathbf{G}^{200}$

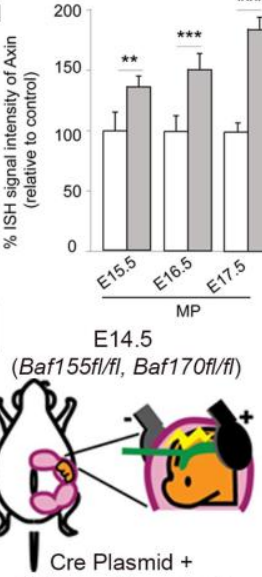

TOP reporter plasmid

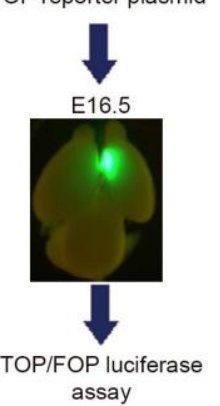

D

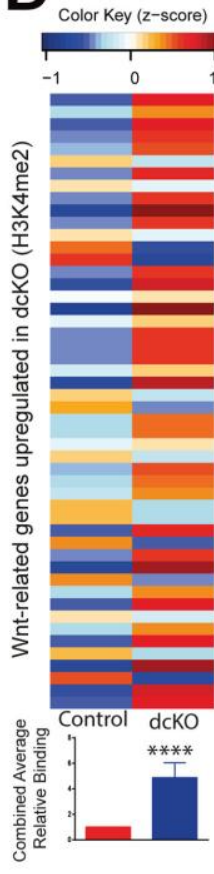

$\square$ Control

$\square$ dcKO_hGFAP-Cre

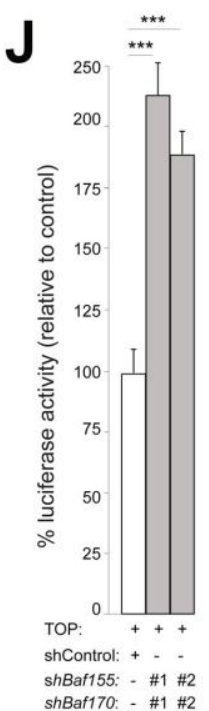




\section{Figure 3.6. BAF complexes suppress Wnt signaling activity.}

(A) Wnt-related genes are upregulated in the dcKO pallium at E17.5. (B) Wnt target genes upregulated in the dcKO pallium are shown. (C) General H3K4me2 profile plot of Wnt-related genes that are upregulated in dcKO pallium. (D) Upper panel: Heatmap depicting the changes in H3K4me2 levels of Wnt-related genes that are upregulated in dcKO pallium at E17.5. Lower panel: Average relative H3K4me2 binding levels on those genes combined. (E) Integrated genome browser views of H3K4me2 and Brg1 binding (GSE37151) (Attanasio et al., 2014) along representative Wnt-related genes upregulated in dcKO pallium. (F-G) ISH $(F)$ and quantitative $(G)$ analyses comparing the expression of the Wnt target Axin2 in the control and dcKO pallium at E15.5-E17.5. $(\mathrm{H}-\mathrm{J})$ In vivo $(\mathrm{H}, \mathrm{I})$ and in vitro $(\mathrm{J})$ luciferase assay indicating higher Wnt signaling activity in BAF155/BAF170-depleted pallial cells (I) and in Neuro2A cells (J) compared to control cells. Values are presented as means \pm SEMs $\left(n>3,{ }^{*} P<0.05,{ }^{* * *} P<0.01,{ }^{* * *} P<0.005\right)$. Abbreviations: TSS, transcription start site; TES, transcription end site; MP, medial pallium; $\mathrm{DP}$, dorsal pallium; LP, lateral pallium. Scale bars $=100 \mu \mathrm{m}(\mathrm{F})$.

To provide additional support, we also performed in situ hybridization (ISH) analysis of the expression of Axin2, a direct target of Wnt/ $\beta$-catenin activity. This analysis showed that, unlike controls, in which Axin2 mRNA staining was faint and confined mostly to the MP at E15.5-E17.5, the BAF complex-deficient pallium exhibited diffused Axin2 staining in the MP VZ at E15.5 and throughout the pallium VZ at E16.5-E17.5 (Figure 3.6F, G).

To further address the capacity of BAF complexes to regulate Wnt-signaling, we performed in vivo reporter assays by electroporating a luciferase promoter construct TOP, containing $\beta$-catenin/TCF binding sites and a mutated form, FOP, as negative control into the embryonic brain. To eliminate BAF function, we electroporated TOP-/FOP-FLASH reporter plasmids plus a Cre-expressing plasmid into the E14.5 MP of $B A F 155^{f / / f l}: B A F 170^{f / / f l}$ embryos. We then examined isolated tissue samples from the MP using the TOP/FOP luciferase assay. These analyses indicated that BAF complex knockout in the pallium significantly enhanced TOP-, but not FOP-reporter activity (Figure 6H, I). Similarly, dual silencing of BAF155 and BAF170 markedly increased Wnt signaling activity in Neuro2A cells in vitro (Figure 6J), suggesting that BAF complex deficiency indeed increased the transcriptional activity of Wnt target genes that control NSC proliferation. Such candidate genes (e.g. Pax6, AP2 $\gamma$ and cyclin D1) are critical for the timely progression of the cell cycle (Figure 3.7A). 
Next, we directly determined whether BAF complex in the MP regulates hippocampal development via suppression of Wnt signaling. To this end, we used ICG-001, a Wnt signaling inhibitor, to perform rescue experiments (Figure 3.7A, B). Starting from E11.5, pregnant mice were intraperitoneally injected daily with an ICG-001 solution, and brain samples were collected at E15.5, E17.5, and P0 (Figure 3.7B). ICG-001 treatment of dcKO mutants resulted in the reversal of NE-like cell characteristics (BLBPlow/GLAST ${ }^{\text {low/occludin }}{ }^{\text {high }}$ ) to $R G$ features (BLBPhigh/GLASThigh/occludin ${ }^{\text {low }}$ ) that typifies the WT pallium (Figure S3.7B-E). Furthermore, IF analyses at E15.5 with Pax6 and Casp3 antibodies revealed that the Wnt inhibition decreased the number of Pax6 $6^{+}$NSCs and $\mathrm{Casp}^{+}$apoptotic cells (Figure 3.7C, E) in dcKO mutants. Concurrently, ICG-001 administration in dcKO mutants caused a near-WT increase in the number of Prox1+ DGs and Ztbt20+ hippocampal neurons in MP (Figure 3.7D, E) and also in the number of Ctip2+, Satb2+, and $\mathrm{Cux}^{+}$cortical neurons in L/DP (Figure S3.7F-H). Strikingly, treatment with the Wnt-inhibitor almost completely rescued the aberrant hippocampal morphology in mutants (Figure 3.7D, E). To consolidate this claim, pregnant mice were treated with XAV-939, a substance with similar effect as ICG-001(Mutch et al., 2010). As expected, XAV-939 treatment reproduced the Wnt inhibition-dependent rescue of cortical anomalies in dcKO mutants (Figure S3.7I-O). 
A

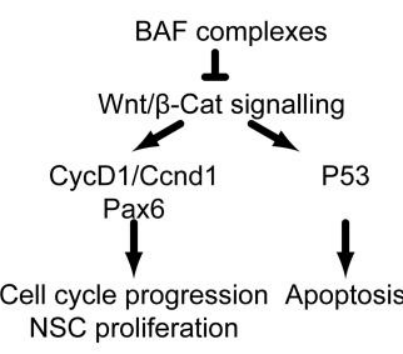

B

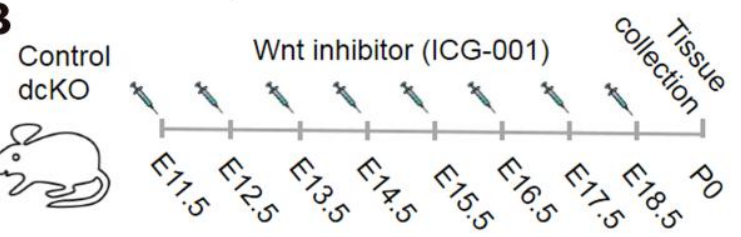

C $\frac{\text { Vehicle }}{\text { Control dcKO }} \frac{\text { Wnt inhibitor }}{\text { dcKO }}$
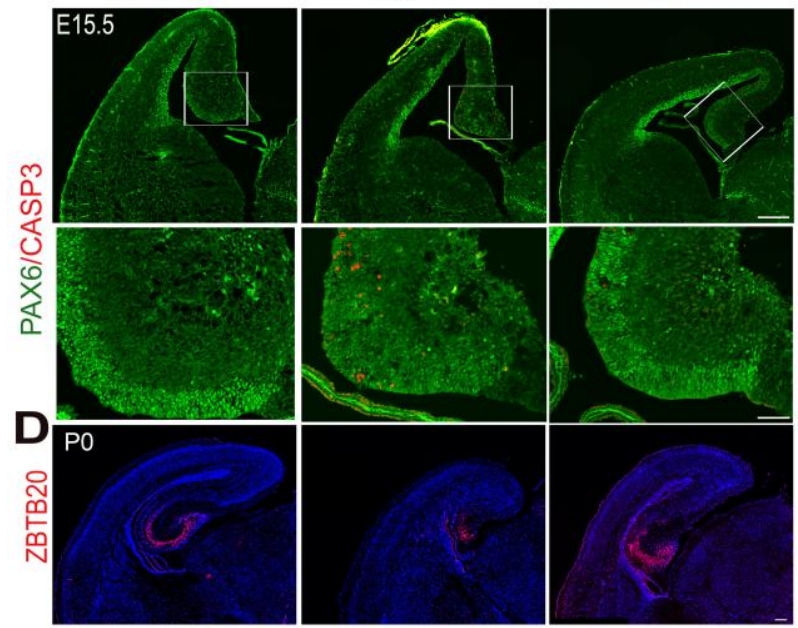

E

$\square$ Control+Veh

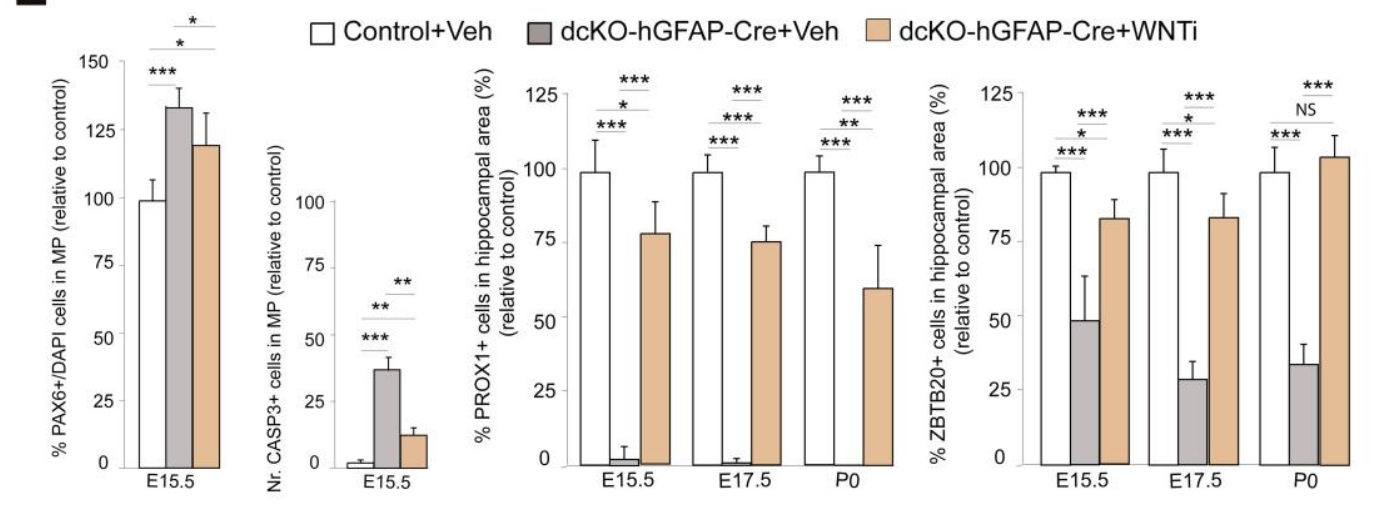

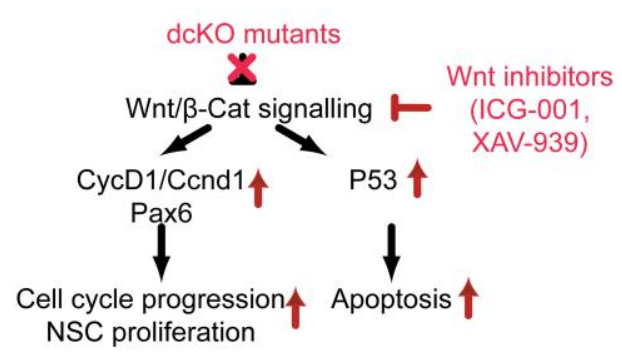

KO mutants

1/Ccnd1个 P53 个

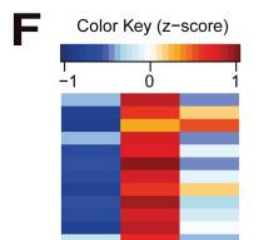

G
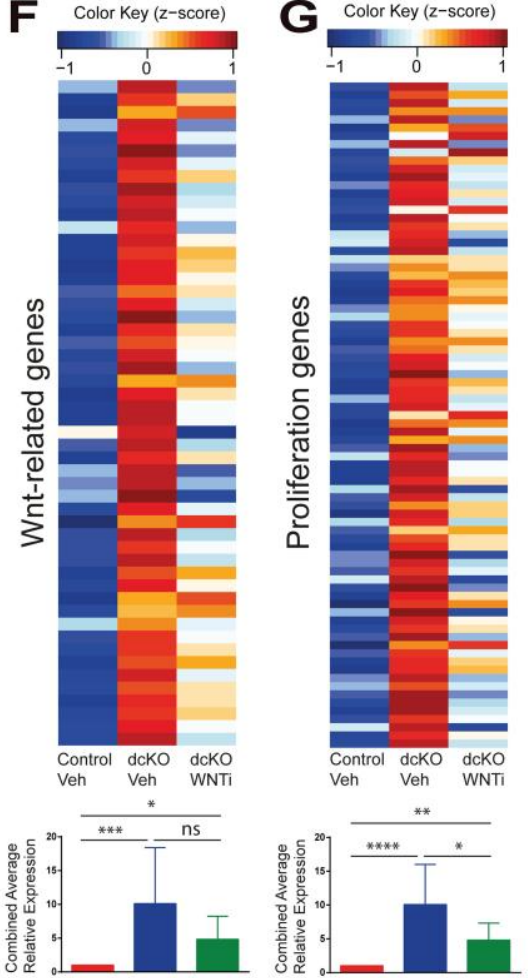

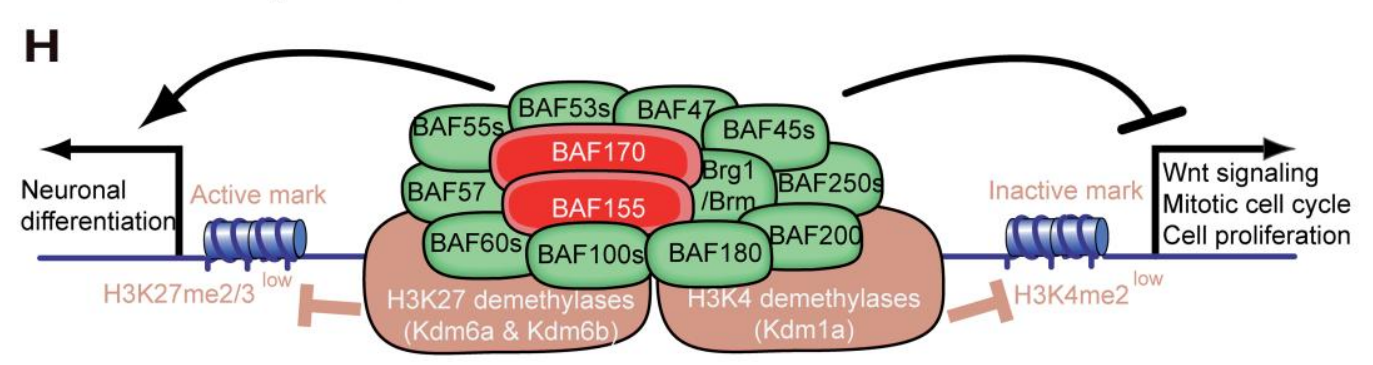


Figure 3.7. BAF complexes control hippocampal development by suppressing Wnt signaling activity.

(A) Schematic model of the molecular cascades underlying late stages of pallium development in WT and dcKO pallium. (B) Rescue experimental paradigm with the Wnt inhibitor (WNTi, ICG-001). IF (C,D) and quantitative (E) analyses of dcKO mutants at the indicated stages, showing the effects of treatment with ICG-001 on pools of Pax6 ${ }^{+} \mathrm{NSCs}$ $(\mathrm{C}, \mathrm{E})$, Casp3 ${ }^{+}$apoptotic cells $(\mathrm{C}, \mathrm{E})$, and $\mathrm{Ztbt20}^{+}(\mathrm{D}, \mathrm{E})$ and Prox1 ${ }^{+}$neurons $(\mathrm{E})$ in the developing hippocampus. $(F, G)$ Expression of Wnt $(F)$ and Proliferation $(G)$-related genes in control, Vehicle (Veh), WNTi-treated pallium. (H) A proposed model showing how loss of BAF155 and BAF170 in dcKO mutants controls epigenetic and neural gene expression programs in proliferation and neuronal differentiation of the pallium in late developmental stages. Values are presented as means \pm SEMs $\left(n>3,{ }^{*} p\right.$-value $<0.05,{ }^{* * *} p$-value $<0.01$, ${ }^{* * *} p$-value $\left.<0.005\right)$. Scale bars $=100 \mu \mathrm{m}(\mathrm{C}, \mathrm{F})$ and $50 \mu \mathrm{m}(\mathrm{C})$.

Finally, we compared the transcriptome of cortices from control and dcKO embryos, which were treated with either Veh or Wnt inhibitor (Figure 3.7F, G). Treatment with Wnt inhibitor decreases the expression of proliferation- and Wnt-related genes that are upregulated in dcKO embryos (Figure 3.7F, G).

Taken together, these results demonstrate that loss of BAF complexes during late cortical neurogenesis leads to aberrant enhancement of Wnt signaling activity and causes increased NSC proliferation-related defects similar to those observed after Wnt/ß-catenin overexpression (Chenn \& Walsh, 2002; Machon et al., 2007). These findings demonstrate that BAF complexes are required for proper hippocampal development through appropriate suppression of Wnt signaling in late developmental stages of the pallium.

\subsection{Discussion}

In this study, we present evidence for the involvement of chromatin remodeling BAF complexes in the regulation of global gene expression and epigenetic programs during late cortical neurogenesis. We showed that specific interactions of BAF155/BAF170 subunits with H3K27 and H3K4 demethylases possibly potentiate their activity during corticogenesis. During late development, loss of H3K27me3 and $\mathrm{H} 3 \mathrm{~K} 4 \mathrm{me} 2$ marks on regulatory regions of distinct sets of genes potentiates disinhibition of transcription of RG- and neuronal differentiation-related genes, and suppresses NE-, 
Wnt signaling-, cell cycle-, and proliferation-related genes, respectively (Figure 3.5I). Thus, BAF complexes act both as activators and as repressors to regulate global epigenetic and gene expression programs during late corticogenesis and hippocampus development.

\subsubsection{BAF155/BAF170-dependent maintenance of $R G$ cell fate during late cortical neurogenesis.}

Cortical neurogenesis comprises three main phases: (1) an expansion phase, characterized by symmetric division of NEs and expansion of the proliferative cell population, (2) a transition period during which NEs differentiate into RGs via asymmetric divisions to generate neurons as well as basal progenitors, and (3) a terminal phase during which progenitors undergo a terminal symmetric division to generate neurons and become quiescent (Gotz \& Huttner, 2005; Dehay \& Kennedy, 2007; Kriegstein \& Alvarez-Buylla, 2009; Martynoga et al., 2012).

A few molecular factors are known to regulate NEs to RGs transition and RGs differentiation (Gotz \& Huttner, 2005; Dehay \& Kennedy, 2007; Kriegstein \& Alvarez-Buylla, 2009; Martynoga et al., 2012). For example, ablation of Fgf10 delayed $R G$ differentiation during early corticogenesis, whereas NE fate seemed unaffected (Sahara \& O'Leary, 2009). It is thus conceivable that at later stages, other mechanisms may be required in limiting NSC fate to allow neuronal differentiation.

We showed that elimination of the BAF complex, by deleting the two scaffolding subunits BAF155 and BAF170 in cortex from E14.5 onward, results in a loss of RG fate hallmarks (diminished expression of astroglial and adherens junction markers), accompanied by gain of NE features (activation of tight junction and proliferation genes) (Figure $3.5 \mathrm{H}$ ). Phenotypically, in the dcKO pallium, we found an overactive progenitor proliferation through symmetric divisions (a feature of NEs), instead of the typical predominant late stage asymmetric division to produce one $R G$ and a neuron or an IP. This leads to overproduction of NSCs at the expense of their derivatives (IPs and UL neurons), and instigating the decreased radial cortical thickness and hypoplasia of the hippocampus in the dcKO mutants (Figure $3.5 \mathrm{H}$ ).

Altogether, our results demonstrate that the chromatin remodeling BAF complex is a crucial factor for ensuring the suppression of NE fate in the late neurogenic phase of corticogenesis. 


\subsubsection{BAF complexes control NSC proliferation and differentiation in early and late embryonic stages via distinct epigenetic mechanisms.}

Discrete histone marks activate or inhibit gene expression programs that regulate neural development. Modifications such as $\mathrm{H} 3 \mathrm{~K} 4 \mathrm{me} 2 / 3$ and $\mathrm{H} 3 \mathrm{~K} 27 \mathrm{Me} 2 / 3$, regulated by their corresponding histone lysine methyltransferases (KMTs) and demethylases (KDMs), are associated with transcriptional activation and repression respectively.

H3K4 is commonly targeted by numerous KMTs and KDMs. In pluripotent ESCs, H3K4me2 marks signaling pathway genes that are required for the transition of neural progenitors to mature neurons (Zhang et al., 2012). H3K4me2 marks are established mainly by KMT2C/D methyltransferases and are removed by the KDM1 (LSD1) demethylase (Shi et al., 2004), which we found to be highly expressed in late cortical progenitors (Figure S3.1). Interestingly, LSD1 is also highly expressed in late progenitors in the developing mouse retina, and its inhibition blocks the differentiation of rod photoreceptors during late developmental stages (Popova et al., 2016).

Our earlier work indicated that BAF complexes interact with the H3K27 demethylases $\mathrm{Kdm6a} / \mathrm{b}$ to promote cell proliferation and neuronal differentiation in early cortical development (Narayanan et al., 2015b). Accordingly, loss of BAF complexes in early corticogenesis results in a global increase in repressive H3K27me3 marks, and downregulation of genes important for progenitor proliferation and differentiation. These two outcomes following ablation of BAF complexes during late development suggest a dual function of BAF complexes in activating neuronal differentiation genes and suppressing proliferation-related pathways that may reflect independent processes mediated by distinct BAF complex cofactors. Like in early stages, BAF complexes possibly induce neuronal differentiation by interacting with $\mathrm{Kdm6a/b}$ to remove inactivating $\mathrm{H} 3 \mathrm{~K} 27 \mathrm{me} 3$ marks on loci of neuronal differentiation genes (Figure 3.7H). In parallel, however, BAF complexes inhibit cell amplification, probably by interacting with $\mathrm{Kdm} 1 \mathrm{a}$ and potentiate its demethylase activity in $\mathrm{H} 3 \mathrm{~K} 4 \mathrm{me} 2$ removal at genomic loci of genes involved in Wnt signaling, mitotic cell cycling, and proliferation (Figure $3.7 \mathrm{H}$ ).

Based on these data, we propose that during late pallium development, endogenous BAF complexes associate with the coactivators $\mathrm{Kdm6a/b}$ to promote neuronal differentiation, while inhibiting cell proliferation via Kdm1a recruitment. 


\subsubsection{BAF complexes suppress Wnt signaling activity}

During cortical neurogenesis, temporal differentiation of NSCs leads to generation of cohorts of neurons with distinct layer identities, a process that depends on multiple regulatory pathways. Wnt signalling regulates the switch between proliferation and differentiation of cortical progenitors. Accodingly, ablation of $\beta$-catenin or Lrp6 (Wnt co-receptor) causes early cell cycle exit and premature differentiation of RGs into IPs and neurons. (Woodhead et al., 2006; Zhou et al., 2006; Machon et al., 2007; Draganova et al., 2015). Conversely, persistent expression of $\beta$-catenin suppresses progenitor exit from mitosis, causing hyper-proliferation of NSCs through excessive symmetric division that consequently delays generation of Tbr2+ IPs and neuronal differentiation in the pallium (Chenn \& Walsh, 2002; Machon et al., 2007; Wrobel et al., 2007; Mutch et al., 2010). Interestingly, these phenotypes are reminiscent of the observed abnormalities in the dcKO cortex.

Previous studies demonstrated that in mammalian non-neural cells, the core BAF subunit Brg1 positively regulates Wnt signaling at distinct levels (e.g. exerting a control of genes encoding for Wnt receptors and also modulating $\beta$-catenin-dependent transcriptional activity) (Barker et al., 2001b; Griffin et al., 2011). Surprisingly, upon loss of BAF155 and BAF170 in late cortical progenitors, multiple components and targets of the canonical $\mathrm{Wnt} / \mathrm{\beta}$-catenin signaling were upregulated, suggesting that the SWI/SNF complex can act to control the Wnt/ $\beta$-cateninn signaling pathway in a tissue- and context-dependent manner. Pharmacological inhibition of $\mathrm{Wnt} / \beta$-catenin signaling rescued the observed defects in cell proliferation, cell survival, and restored hippocampal morphology in the dcKO mutants, hence making us posit that BAF (SWI/SNF) complexes negatively regulate Wnt signaling during late cortical neurogenesis.

Altogether, our results indicate that the chromatin remodeler BAF plays a crucial role in late-stage development of mammalian cortex in two distinct ways. On one hand, BAF complexes induce heterochromatin formation at loci of cell cycle, proliferation and Wnt-related genes, thereby suppressing their expression; and the other, they facilitate the expression of neural differentiation-related genes by establishing euchromatin at related genomic regions. Together, these activities ensure the generation of appropriate numbers of NSCs and neurons in late cortical development. 


\subsection{Materials and Methods}

\subsubsection{Materials}

\section{Transgenic mice}

$B A F 155^{f / f}$ (Choi et al., 2012), BAF170f/f (Tran et al., 2013), Emx1-Cre (Gorski et al., 2002), hGFAP-Cre (Zhuo et al., 2001), Nex-Cre (Goebbels et al., 2006) and Rosa-tdTomato (Ai9) (Madisen et al., 2010) mice were maintained in a C57BL6/J background. Animals were handled in accordance with the German Animal Protection Law.

\section{Plasmids}

Plasmids used in this study: pCIG2-ires-eGFP, pCIG2-Cre-ires-eGFP (gift from Dr Francois Guillemot, NIMR London); 8XTOPFLASH (TOP) (Veeman et al., 2003) and Super8XFOPFLASH (FOP) (Veeman et al., 2003) were gifts from Randall Moon (Addgene plasmid \# 12456, 12457).

\section{Antibodies}

The following polyclonal ( $\mathrm{pAb}$ ) and monoclonal (mAb) primary antibodies used in this study were obtained from the indicated commercial sources: AP2 $\gamma$ mouse mAb (1:100; Abcam), BAF170 rabbit pAb (Bethyl), BAF170 rabbit pAb (Sigma), BAF155 rabbit pAb (1:20; Santa Cruz), BAF155 mouse mAb (1:100; Santa Cruz), Brn2 goat pAb (1:100; Santa Cruz), BLBP rabbit pAb (1:200; Chemicon), Casp3 rabbit pAb (1:100; Cell Signaling), Ctip2 rat pAb (1:200; Abcam), GLAST pig pAb (1:500; Frontier), CidU rat pAb (1:100; Accurate), H3K27me3 rabbit pAb (Upstate), Kdm6a/Utx rabbit pAb (Santa Cruz), Kdm6b/jmjd3 rabbit pAb (De Santa et al., 2007), Ki67 rabbit pAb (1:50; Vector), HuCD mouse mAb (1:20; Invitrogen), LSD1 rabbit pAb (1:100; Cell Signaling), Satb2 mouse mAb (1:200; Abcam), Sox2 mouse mAb (1:100; R\&D Systems), Prox1 rabbit pAb (1:1000; Covance), Pax6 mouse mAb (1:100; Developmental Studies Hybridoma Bank), Pax6 rabbit pAb (1:200; Covance), Flag mouse mAb (1:1000; Sigma), phospho-H3 rabbit mAb (1:200; Millipore), phospho-H3 rat pAb (1:300; Abcam), pVim mouse mAb (1:500; MBL), occludin rabbit pAb (1:50; Thermo Fisher), TUBB3 mouse mAb (Tuj1, 1:500; Chemicon), Tbr2 rabbit pAb (1:200; Abcam), Tbr1 rabbit pAb (1:300; Chemicon), Zbtb20 rabbit pAb (1:50; Sigma), and RFP rabbit pAb (1:10000; Biomol/Rockland). 
Secondary antibodies used were horseradish peroxidase (HRP)-conjugated goat anti-rabbit IgG (1:10000; Covance), HRP-conjugated goat anti-mouse IgG (1:5000; Covance), HRP-conjugated goat anti-rat IgG (1:10000; Covance), and Alexa 488-, Alexa 568-, Alexa 594- and Alexa 647-conjugated IgG (various species, 1:400; Molecular Probes).

\subsubsection{Methods}

\subsubsection{Generation of dcKO mutants}

To eliminate BAF155 and BAF170 in early cortical progenitors, late cortical progenitors, or projection neurons, we used the early progenitor-active Emx1-Cre (Gorski et al., 2002), late progenitor-active hGFAP-Cre (Zhuo et al., 2001) and neuron-specific Nex-Cre (Goebbels et al., 2006) mouse lines, respectively. Heterozygous animals $\left(B A F 155^{f /+}, B A F 170^{f /+}, C r e\right)$ were used as controls. Mutants crossed with Emx1-Cre or hGFAP-Cre died soon after birth.

\subsubsection{Mass spectrometry, CoIP, ChIP-Seq, and RNA-Seq Chromatin immunoprecipitation (ChIP)}

ChIP assays performed on the pallium from control $(\mathrm{n}=4)$ and dcKO_hGFAP-Cre E17.5 $(n=4)$ littermate embryos. Briefly, tissues were homogenized in sucrose solution (0.32 M sucrose, $5 \mathrm{mM} \mathrm{Cacl} 2,5 \mathrm{mM} \mathrm{Mg(Ac)}$, $0.1 \mathrm{mM}$ EDTA, $50 \mathrm{mM}$ HEPES pH 8, $1 \mathrm{mM}$ DTT, $0.1 \%$ Triton X-100), and then fixed in 37\% formaldehyde. After stopping fixation by adding $1.25 \mathrm{M}$ glycine, samples were washed with Nelson buffer (140 mM NaCl, 20 mM EDTA pH 8, 50 mM Tris pH 8, 0.5\% NP-40, 1\% Triton X-100) and sonicated in RIPA buffer (140 mM NaCl, 1 mM EDTA, 1\% Triton X-100, 0.1\% sodium deoxycholate, $10 \mathrm{mM}$ Tris $\mathrm{pH} 8,1 \%$ SDS).

For ChIP against histone marks $500 \mathrm{ng}$ of chromatin with either $4 \mu \mathrm{g}$ of anti-H3K4me2 antibody (Millipore) or $2 \mu \mathrm{g}$ of anti-H3K27me3 antibody (Millipore) were used for each experiment, with input DNA of $50 \mathrm{ng}$. For ChIP against histone demethylases $10 \mu \mathrm{g}$ of chromatin with either $10 \mu \mathrm{g}$ of anti-LSD1 antibody (Abcam) or $10 \mu \mathrm{g}$ of anti-JMJD3 antibody (Abcam) were used for each experiment with input DNA 
of $100 \mathrm{ng}$. After incubating overnight, samples were incubated with $15 \mu \mathrm{l}$ of protein A-coated beads (Diagenode) that had been blocked by incubating with $0.5 \%$ bovine serum albumen (BSA). Beads were washed with IP buffer $(140 \mathrm{mM} \mathrm{NaCl}$, $1 \%$ NP-40, 0.5\% sodium deoxycholate, $50 \mathrm{mM}$ Tris pH 8, $20 \mathrm{mM}$ EDTA, 0.1\% SDS) and wash buffer $(100 \mathrm{mM}$ Tris $\mathrm{pH} 8,500 \mathrm{mM} \mathrm{LiCl}, 1 \% \mathrm{NP}-40,1 \%$ sodium deoxycholate, $20 \mathrm{mM}$ EDTA), and chromatin was eluted with $0.1 \mu \mathrm{g} / \mu \mathrm{l}$ of RNase A diluted in $10 \mathrm{mM}$ Tris $(\mathrm{pH} \mathrm{8)}$. De-crosslinking was carried out in Wiemann buffer (100 mM Tris pH 8, 20 mM EDTA, 2\% SDS) with Proteinase $\mathrm{K}(1 \mu \mathrm{g} / \mu \mathrm{l})$. DNA was eluted in $10 \mathrm{mM}$ Tris $(\mathrm{pH} 8)$.

\section{ChIP-Sequencing}

Libraries were prepared with a NEBNext Ultra DNA Library Prep Kit for Illumina (NEB) and analyzed using QuBit and an Agilent 2100 Bioanalyzer. Input DNA (50 ng) was isolated from each sample and then pooled separately for each group.

Base calling and FASTQ conversions were performed using standard Illumina scripts, as described previously (Halder et al., 2016; Narayanan et al., 2015). Quality control was also performed for each sample using FastQC (www.bioinformatics.babraham.ac.uk/projects/fastqc). Reads were mapped to the mm10 mouse reference genome using STAR aligner v2.3.0 (Djebali et al., 2012). BAM files were filtered leaving only high-quality reads $[M A P Q !=(0,2,3,4)]$ as described previously (Halder et al., 2016).

BAM files of replicates from the same group were combined using the merge function of SAMTools ( $\mathrm{Li}$ et al., 2009). Genomic profile plots were created from combined BAM files using NGSPlot (Shen et al., 2014). Wiggle (WIG) files were created from these BAM files using the script available in the MEDIPS package of Bioconductor (Lienhard et al., 2014). Visualization of individual gene loci was performed with Integrated Genome Browser (Nicol et al., 2009) using these WIG files.

Peaks were called using MACS2, with $q<0.1$ (Feng et al., 2012). Differential binding analyses were performed using the DiffBind package of Bioconductor (Ross-Innes et al., 2012) with the DESEQ2 option for differential 
analysis. Peak annotation was performed using HOMER (Heinz et al., 2010) and homemade scripts.

\section{RNA-Sequencing}

RNA was extracted (RNeasy kit; Qiagen) from the pallium of control ( $n=4)$ and dcKO_hGFAP-Cre $(\mathrm{n}=3)$ E17.5 littermate embryos. cDNA libraries were prepared using the TruSeq RNA Sample Preparation v2 Kit. DNA was quantified using a Nanodrop spectrophotometer, and its quality was assessed using an Agilent 2100 Bioanalyzer.

Base calling, fastq conversion, quality control, and read alignments were all performed as outlined for ChIP-Seq. Reads were aligned to mouse genome mm10 and counted using FeaturesCount (http://bioinf.wehi.edu.au/featureCounts/). Differential expression was assessed using DESeq2 from Bioconductor (Love et al., 2014). Functional GO enrichment analyses were performed using ToppGene (Chen et al., 2009).

The high-throughput RNA-seq and ChIP-Seq data will be deposited in the NCBI Gene Expression Omnibus and made accessible through GEO Series accession numbers upon acceptance of the manuscript.

\subsubsection{3. qPCR, ChIP-qPCR}

Briefly, qPCR for confirmation of gene expression changes cDNA was synthesized using Transcriptor High Fidelity cDNA Synthesis Kit (Roche) and the results were normalized to housekeeping gene hypoxanthine phosphoribosyltransferase (Hprt1). For ChIP-qPCR the results were normalized against input DNA. All qPCR reactions were performed in Roche 480 Light Cycler using SYBR Green. To validate the upregulated expression of Wnt-related genes in our RNA-Seq dataset, we performed qPCR using the mouse WNT Signaling Pathway RT2 Profiler PCR Array (PAMM-043Z; Qiagen) and mouse WNT Signaling Targets RT2 Profiler PCR Array (PAMM-243Z; Qiagen).

\subsubsection{Co-immunoprecipitation and mass spectrometry (CoIP/MS)}


BAF155 and BAF170 interaction analyses were performed using the neural stem cell line, NS5 (Conti et al., 2005), and E13.5 and E17.5 embryonic telencephalic tissue. Tissues were dissected and minced in cold phosphate-buffered saline (PBS) and then washed twice with PBS. Equivalent amounts of cells from one embryo were lysed for $30 \mathrm{~min}$ in $1 \mathrm{ml}$ RIPA buffer containing a proteinase inhibitor cocktail (Roche) and DNase. All steps were performed at $4^{\circ} \mathrm{C}$. Lysates were centrifuged for $10 \mathrm{~min}$ at $13,000 \mathrm{rpm}$ to sediment out non-lysed tissues. The supernatant was pre-cleared by incubating with normal mouse IgG together with protein $A / G$-agarose beads, as described by the manufacturer (sc-2003; Santa Cruz). Interacting proteins were immunoprecipitated by incubating pre-cleared supernatant with rabbit anti-BAF155 and anti-BAF170 antibodies and A/G-agarose beads. The beads were then washed first with $500 \mu \mathrm{l}$ cold RIPA buffer (three times for 5 min each) and then with $40 \mu \mathrm{l}$ of elution buffer $\left(2.5 \mu \mathrm{l} 20 \% \mathrm{SDS}, 5 \mu \mathrm{l} 1 \mathrm{M} \mathrm{NaHCO}_{3}, 42.5 \mu\right.$ l double-distilled $\left.\mathrm{H}_{2} \mathrm{O}\right)$ for $15 \mathrm{~min}$ at room temperature.

For MS analyses (performed in the department of Prof. Dr. Henning Urlaub), samples were suspended in NuPage loading buffer and resolved on commercial SDS polyacrylamide gels (Novex NuPage Bis-Tris gel, 4-12\% gradient; Invitrogen). Individual lanes were then cut into six squares for MS analysis. The parameters for the identification of proteins were set to the following values: limit, 95\% probability of detection; limit of unique peptides detected, 1; and threshold detection probability of peptides, $80 \%$.

The list of BAF155- and BAF170-interacting proteins revealed by MS analysis was obtained by subtracting nonspecific interactions with $\lg G$ in IPs and in telencephalic tissues from BAF155-null (BAF155cKO_FoxG1-Cre) and BAF170-null (BAF170cKO_FoxG1-Cre) mutants. The first set of controls excludes nonspecific binding to the antibody, and the second excludes nonspecific interactions that possibly could be precipitated by either the anti-BAF155 or anti-BAF170 antibody.

\subsubsection{Injection of Wnt inhibitor (WNTi, ICG 001, XAV-939) and Wnt activator (WNTa, SB-216763), H3K27 demethylase inhibitor (GSK-J4), LSD1 histone demethylase (2-PCPA).}


ICG001 (Tocris Bioscience, Cat. No. 4505), XAV-939 (Tocris Bioscience, Cat. No. 3748), SB-216763 (Tocris Bioscience, Cat. No. 1616), GSK-J4 (SIGMA, Cat. No. SML0701) and 2-PCPA (Tocris Bioscience, Cat. No. 3852) were dissolved in vehicle (DMSO). 11.5 d.p.c. pregnant mice received daily injections of vehicle $(150 \mu \mathrm{l})$, or ICG001 $(150 \mu \mathrm{l}$ of a $1-\mathrm{mg} / \mathrm{ml}$ solution), XAV-939, $(150 \mu \mathrm{l}$ of a $0.2 \mathrm{mg} / \mathrm{ml}$ solution), SB-216763 (100 $\mu$ l of a $1-\mathrm{mg} / \mathrm{ml}$ solution plus $100 \mu \mathrm{l}$ of saline), GSK-J4 $(150 \mu \mathrm{l}$ of $2.5 \mathrm{mg} / \mathrm{ml}$ solution)) and 2-PCPA $(150 \mu \mathrm{l}$ of $5 \mathrm{mg} / \mathrm{ml}$ solution). Treated mice were sacrificed at different developmental stages as indicated in the text.

\subsubsection{In vivo $\beta$-catenin transcriptional activity assay}

In vivo and in vitro $\beta$-catenin transcriptional activity assay were performed as previously described (Mao et al., 2009; Durak et al., 2016). Briefly, Wnt/ß-catenin transcriptional activity in vivo was monitored by electroporating brains of E13.5 $B A F 155^{1 / / / 1 /} ; B A F 170^{f / / f l}$ embryos with a Cre plasmid (or empty plasmid as a control) and the reporter constructs Super8XTOPFLASH (TOP) or Super8XFOPFLASH (FOP) together with $p R L-T K$ constructs at a 5:1:0.3 ratio. $\mathrm{Wnt} / \beta$-catenin transcriptional activity was measured at E15.5. For in vitro assay, Neuro2A cells at $1 \times 10^{5}$ per well density were plated into 24 -well plates. Cells were transfected with $0.8 \mu \mathrm{g}$ of shRNA plasmids (shBAF155, shBAF170) along with $50 \mathrm{ng}$ of Super8XTOPFLASH and $10 \mathrm{ng}$ of pRL-TK. 2 days post-transfection, cells were collected for $\mathrm{Wnt} / \beta$-catenin transcriptional activity measurement. In all cases, firefly luciferase activity was normalized to that of Renilla luciferase.

\subsubsection{Spindle angle analysis}

Brain sections were stained with PVIM to outline the cell shape and PHH3 to identify anaphase and early telophase dividing cells. Images of z-stack sections were obtained by SP5 confocal microscopy, and 3D reconstruction of the confocal stacks was done as described previously (Postiglione et al., 2011; Tran et al., 2013).

\subsubsection{Imaging, quantification, Statistical Analysis and Data availability}

Images were captured by confocal fluorescence microscopy (TCS SP5, Leica) and analyzed using an Axio Imager M2 (Zeiss) with a Neurolucida system. 
Images were further processed with Adobe Photoshop. IF signal intensities were quantified using ImageJ software. The statistic quantification was carried out as average from at least three biological replicates. All RNA-Seq and ChIP-Seq data have been deposited in GEO under accession number GSE106711.

\section{Cell counts and quantitative analysis of IF signal intensity}

Immunostaining in IF images was quantified using anatomically matched forebrain sections. Nucleus-marker-positive cells within the pallium of confocal images were counted for comparison. In most cases, cell counts of six matched sections were averaged from three biological replicates (control/dcKO pallium). In many cases, the number of lineage marker cells was quantified using total marker-positive cells alone, or by normalizing to the total number of $\mathrm{DAPI}^{+}$(nucleus-stained) cells using the following equation: Normalized number $=$ marker-positive cell number/DAPI ${ }^{+}$cell number. Statistical analyses of histological data were performed using Student's $t$-test. All bar graphs are plotted as means \pm SEM. All statistical tests are two-tailed, and $P$-values are considered to be significant for $\alpha=0.05$.

\section{Statistical Analysis}

Statistical analyses were designed using the assumption of normal distribution and similar variance among groups, as previously tested. The sample size was determined based on preliminary results or similar experiments carried-out in the past. Power analysis was performed using G-power in order to estimate the number of animals required, for a signal-to-noise ratio of 1.4 and $80 \%$ to $90 \%$ power assuming a $5 \%$ significance level. For histological analysis, qPCR, ChIP-qPCR and comparison of genome-wide histone marks, statistics were assessed with Student's $t$-test. The significance of overlaps was assessed using hypergeometric test. RNA-Seq and ChIP-Seq analyses were carried out using DESeq2 and DiffBind packages of Bioconductor respectively, with their own in-built statistical calculation tools. The results are presented as means \pm SEM. $P$ values of $<0.05$ were considered to be statistically significant unless otherwise indicated. All the relevant 
information pertaining to statistical analysis is also specified in each figure legend separately. The statistic quantification was carried out as average from at least three biological replicates. 
Chapter 3

\section{Supplemental figures}

A

Wild-type
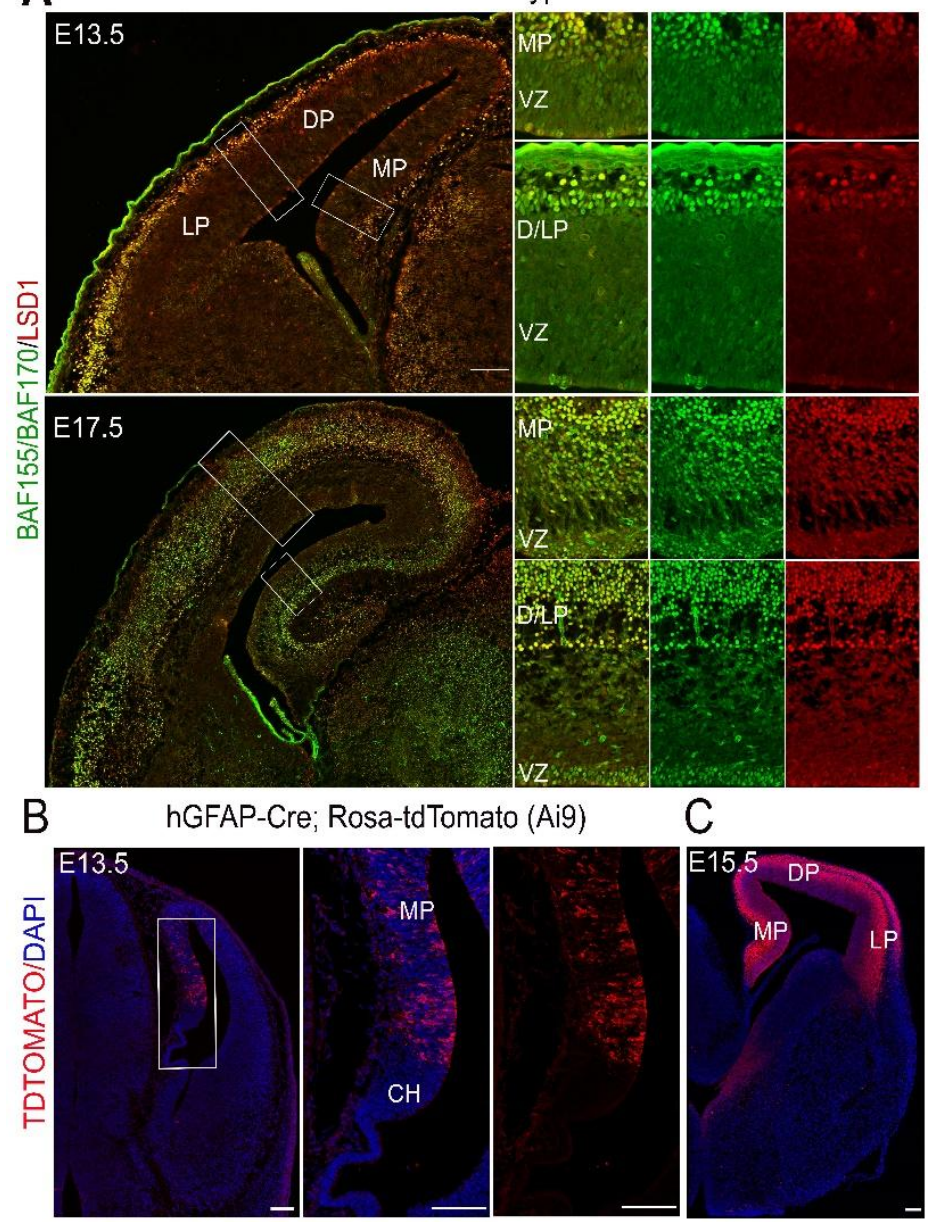

D BAF155/BAF170

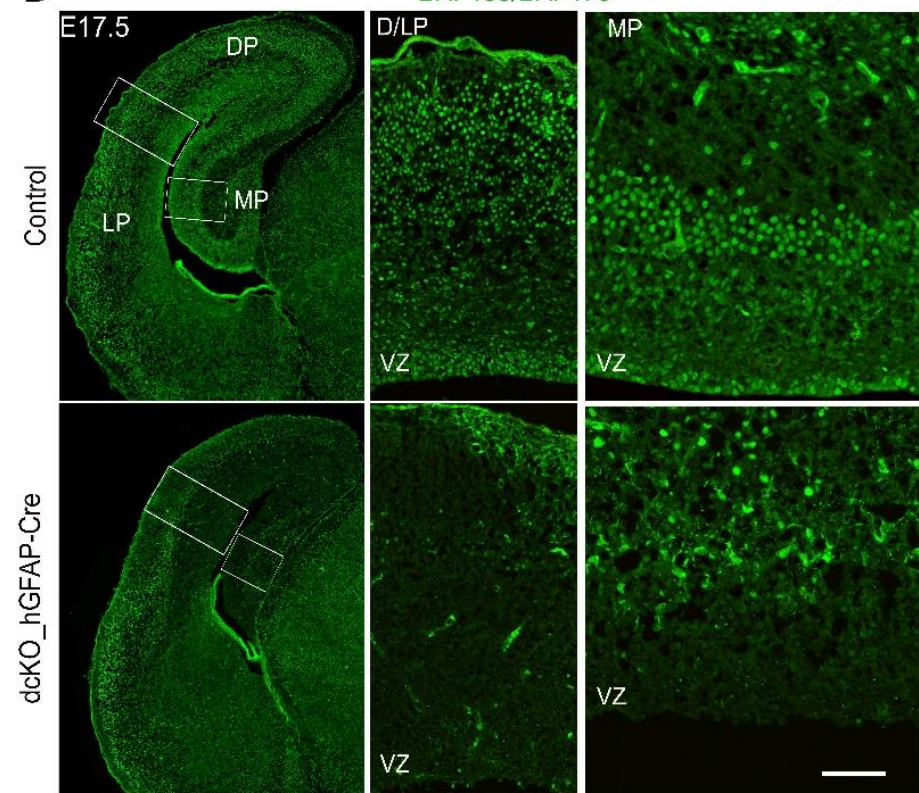


Figure S3.1 (related to figure 1). Co-expression of BAF155/BAF170 with H3K4 and H3K27 demethylases in the developing pallium; hGFAP-Cre activity in the developing pallium; and expression of BAF155/BAF170 in the dcKO_hGFAP-Cre pallium. (A) Double-label IF analysis with anti-BAF155/BAF170 (green) and anti-LSD1/Kdm1a (red) antibodies, showing co-expression of BAF155/BAF170 with LSD1/Kdm1a in pallium cells. Co-expression of BAF155/BAF170 with UTX/Kdm6a or JMJD3/ Kdm6b in the developing forebrain was analyzed previously (Narayanan et al.). (B, C) Immunostaining for dtTomato on cortical sections from hGFAP-Cre; Rosa-tdTomato (Ai9) embryos at E13.5 (B) and E15.5 (C). (B) The right panels are higher-magnification images from the fields in the MP indicated by white frames. By E13.5, recombination is restricted in MP but absent from D/LP and cortical hem (CH) (B). From E15.5 onward, Cre recombination was detected in entire pallium (C). (D) Compared with controls, expression of BAF155 and BAF170 was largely lost in the dcKO pallium, as shown by IF analysis with anti-BAF155/BAF170 antibodies. Abbreviations: VZ, ventricular zone; MP, medial pallium; DP, dorsal pallium; LP, lateral pallium; $\mathrm{CH}$, cortical hem. Scale bars $=100 \mu \mathrm{m}$. 
A E17.5 Control E17.5 dcKO_hGFAP-Cre qPCR (Differentiation Genes)
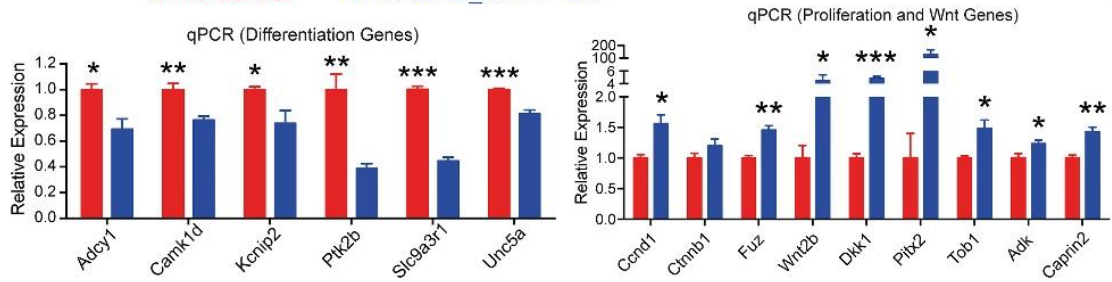

H3K4me2 ChIP-qPCR (Proliferation and Wnt Genes)

B

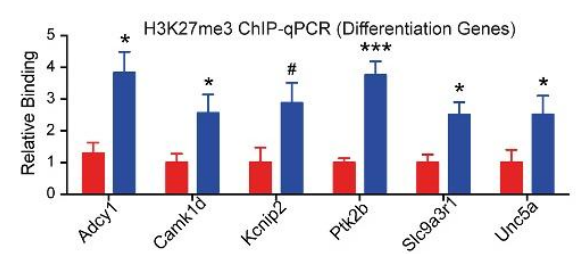

C

Genes are bound by Brg1

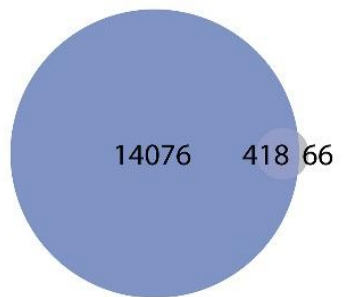

Genes with the increased $\mathrm{H}_{3} \mathrm{~K} 27 \mathrm{me} 3$ in dcKO_hGFAP-Cre pallium

Genes bound by Brg1

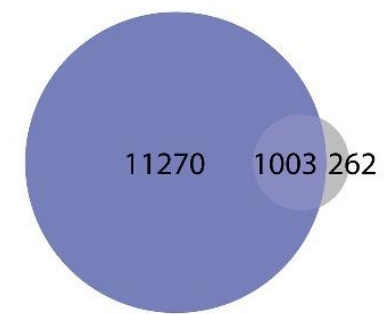

Genes with the increased H3K4me2 in dcKO_hGFAP-Cre pallium

E
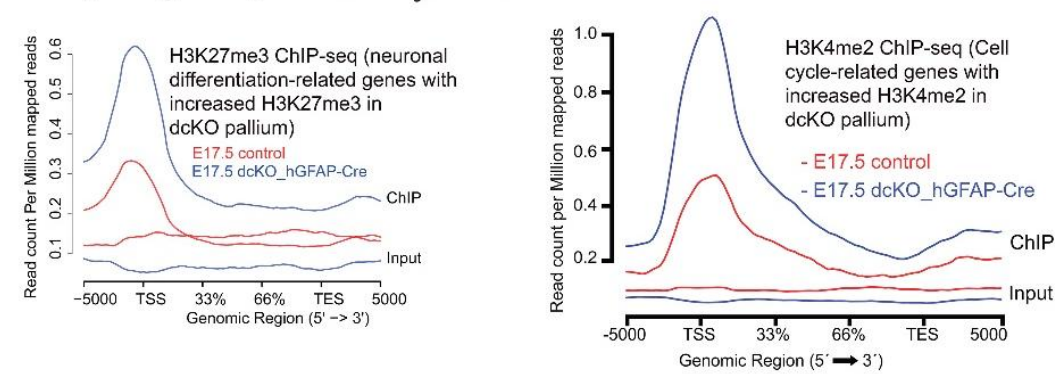

Neuronal differentiation-related pathways with increased H3K27me3 in Mutants: $\square$ dcKO_hGFAP-Cre vs Control (H3K27me3 ChIP-seq)

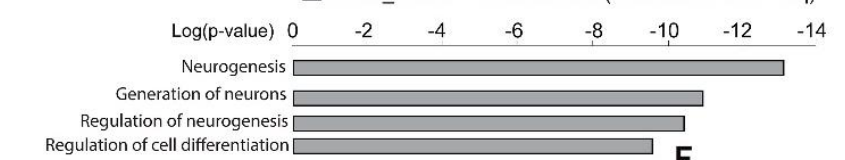
Regulation of neurogenesis

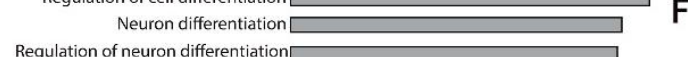

Regulation of neuron differentiation

Regulation of cell morphogenesis

Cell projection organization Neuron development

gulation of cell morphogenesis involved in differentiation

Regulation of neuron projection development

Regulation of cell projection organization

Cellmorpho

hogenesis involved in differentiation

Neuron projection morphogenesis

Regulation of axonogenesis

ved in neuron differentiation

axonogenesis

Cell cycle-related pathways

with increased $\mathrm{H} 3 \mathrm{~K} 4 \mathrm{me} 2$ in Mutants:

$\square$ dcKO_hGFAP-Cre vs Control (ChIP-seq)

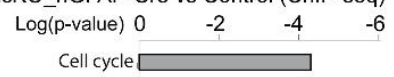

Mitotic cell cycle $\square$

Negative regulation of phase transition $\square$

Negative regulation of mitotic cell cycle $\square$ Cell cycle process

Cell cycle checkpoint

Regulation of cell cycle
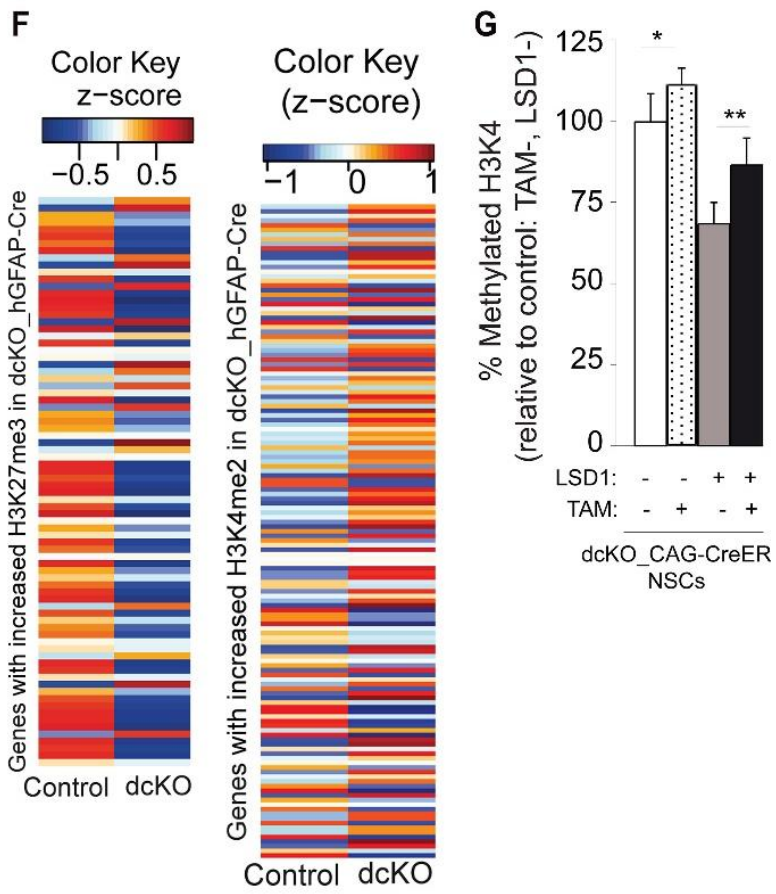

Regulation of mitotic cell cycle

Negative regulation of cell cycle 
Figure S3.2 (related to figures 1, 2, 4). Characterization of H3K27me3 and H3K4me2 marks in dcKO_hGFAP-Cre cortex. (A) qPCR confirmation of selected neuronal differentiation-related genes downregulated, proliferation- and Wnt-related genes upregulated in dcKO_hGFAP-Cre embryos at E17.5 (Control: $n=4$, dcKO: $n=4$ ). Student's t-test: *** $p$-value $<0.001,{ }^{* *} p$-value $<0.01,{ }^{*} p$-value $<0.05$. (B) ChIP-qPCR confirmation of H3K27me3 levels at selected neuronal differentiation-related genes downregulated and H3K4me2 levels at selected proliferation and Wnt-related genes upregulated in dcKO_hGFAP-Cre embryos at E17.5 (Control: $n=4$, dcKO: $n=4$ ). Student's t-test: ${ }^{* * *} p$-value $<0.001,{ }^{* *} p$-value $<0.01,{ }^{*} p$-value $<0.05$, \# $p$-value $<0.1$. (C) Overlap between genes bound by Brg1 (GSE37151; Attanasio et al., 2014) and those with increased H3K27me3 and those with increased H3K4me2 in dcKO embryos (hypergeometric test: $p$-value < 0.0001 ). (D) General profile plots of H3K27me3 at neuronal differentiation-related genes with increased H3K27me3 and H3K4me2 at cell cycle-related genes with increased H3K4me2 in dcKO_hGFAP-Cre embryos ( $p$-value $<0.0001$, Student's t-test). (E) Neuronal differentiation-related genes have increased H3K27me3 and Cell cycle-related genes have increased H3K4me2 ( $p$-value < 0.01) in dcKO_hGFAP-Cre embryos at E17.5. (F) Heatmaps depicting the expression changes in neural differentiation-related genes that have increased H3K27me3 and in cell cycle-related genes that have increased H3K4me2 in dcKO_hGFAP-Cre embryos at E17.5. (G) In the LSD1 demethylase activity quantification assay, cultured dcKO_CAG-Cre NSCs were nucleofected with a mammalian expression vector for LSD1/Kdm1a. The Cre-mediated deletion of BAF155 and BAF170 alleles was induced by adding TAM. Compared to control (LSD1-, TAM-, white bar), overexpression of LSD1 (LSD1+, TAM-, grey bar) decreased the methylated H3K4. In absence of BAF complexes (LSD1+, TAM, black bar) LSD1 displayed its low H3K4 demethylase activity. 
Chapter 3

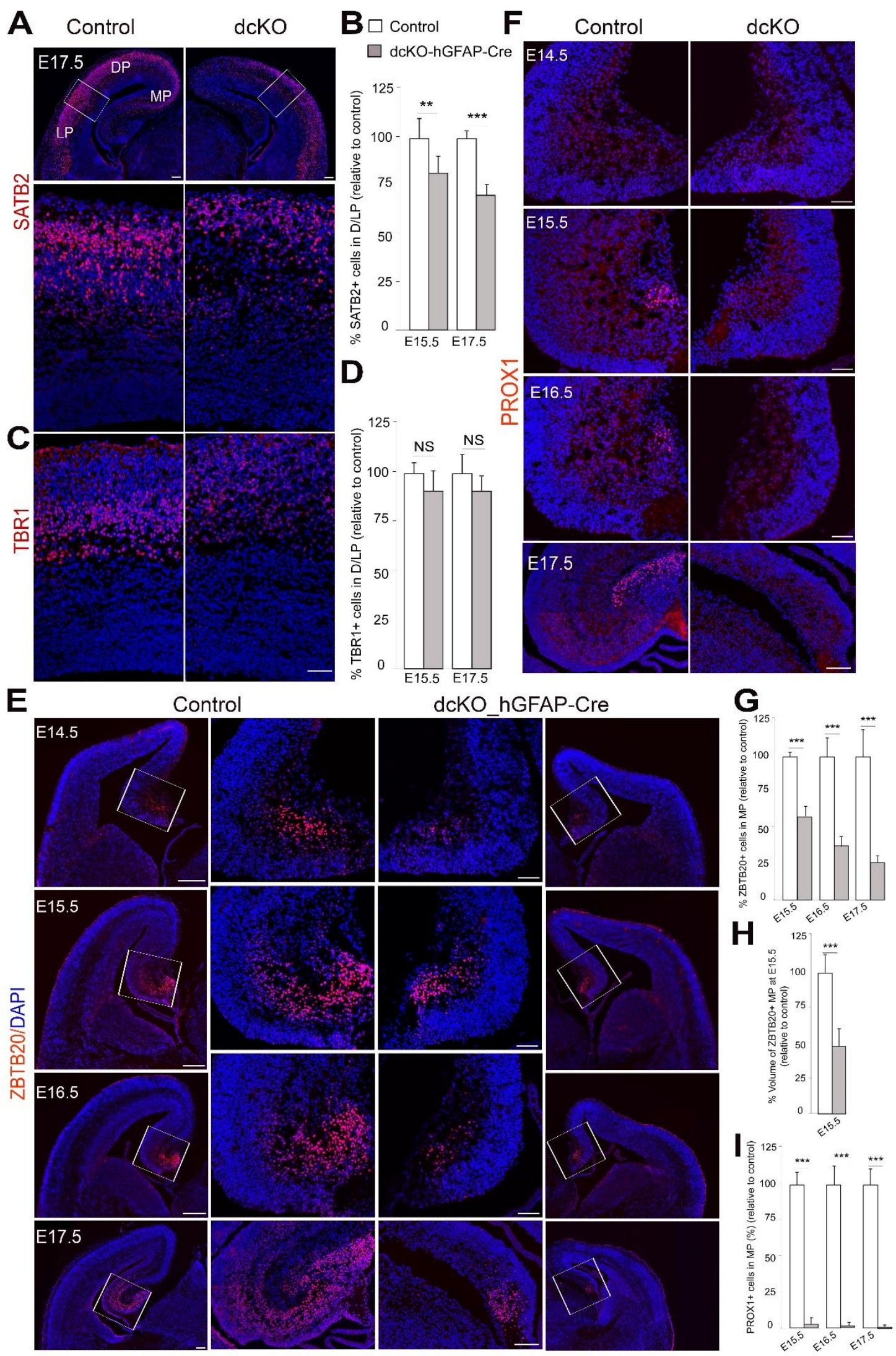


Figure S3.3 (related to figures 2, 3). BAF155 and BAF170 are essential for neurogenesis in the developing cortex and hippocampus. $(A-B)$ IF $(A, C)$ and quantitative $(B, D)$ analyses of the neurogenesis phenotype using the late-born neuronal marker Satb2 (A, B) and early-born neuronal marker Tbr1 (C, D). (E - I) IF (E, F) and quantitative (G-I) analyses for the neuronal markers Ztbt20 in the hippocampus (E, G, H) and Prox1 in the dentate gyrus $(F, I)$ revealed that hippocampal sections of the MP of mutants have a reduced number of $Z$ tbt20+ neurons and Prox1+ neurons at E15.5-E17.5 compared with controls. (H) Quantification of the Ztbt20 ${ }^{+}$hippocampal volume performed across the entire hippocampus using $3 \mathrm{D}$ reconstruction (see also Figure S5A). Values are expressed as means \pm SEMs $\left({ }^{*} P<0.05\right.$; $\left.{ }^{* \star} P<0.01 ;{ }^{* \star *} P<0.005\right)$. Abbreviations: MP, medial pallium; DP, dorsal pallium; $\mathrm{LP}$, lateral pallium. Scale bars $=100 \mu \mathrm{m}(10 \mathrm{x} ; \mathrm{A}, \mathrm{E}), 50 \mu \mathrm{m}(25 \mathrm{x} ; \mathrm{A}, \mathrm{C})$, and $50 \mu \mathrm{m}(40 \mathrm{x} ; \mathrm{E}, \mathrm{F})$. 
A

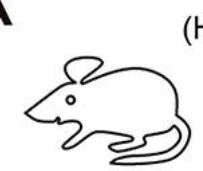

WT:

B

3 demethylase inhibitors)
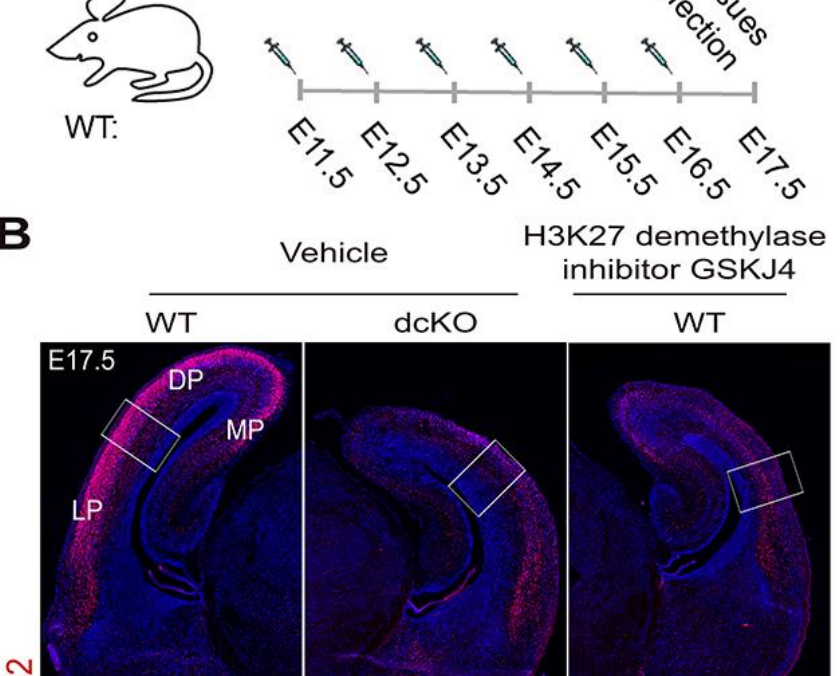

政
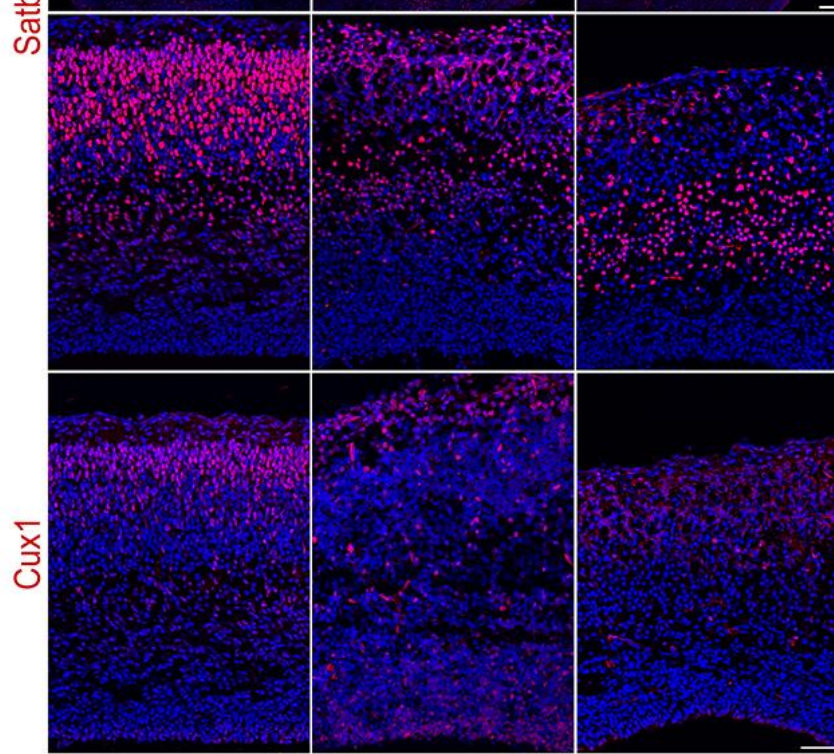

E

Vehicle

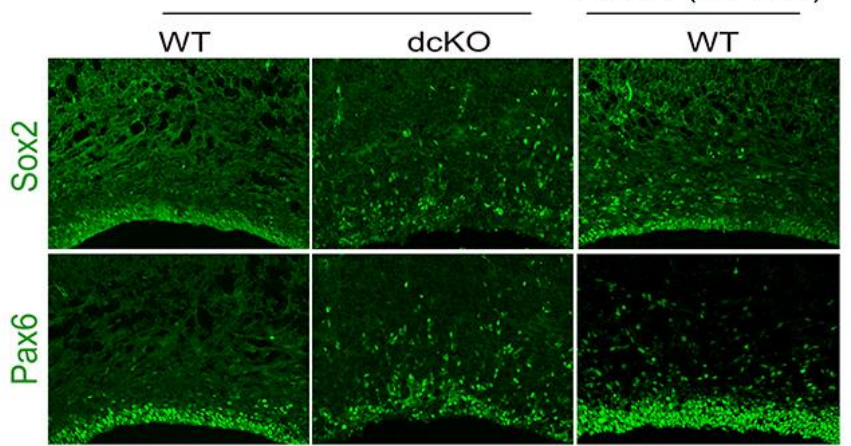

C

$\square \mathrm{WT}+\mathrm{Veh}$

$\square$ dcKO + Veh

$\square$ WT + GSK-J4

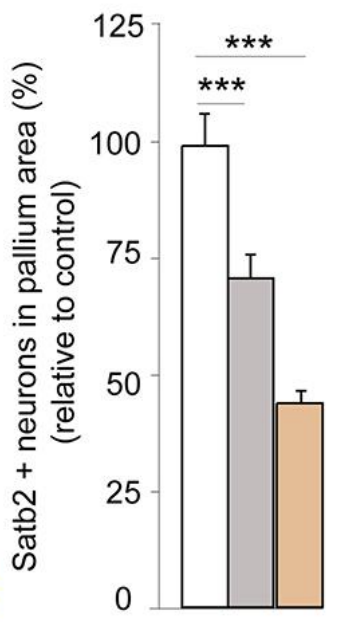

D

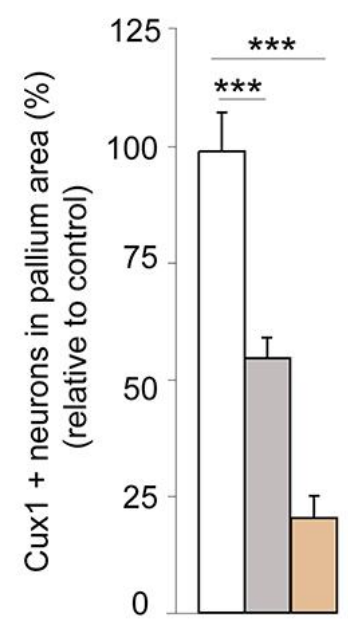

F
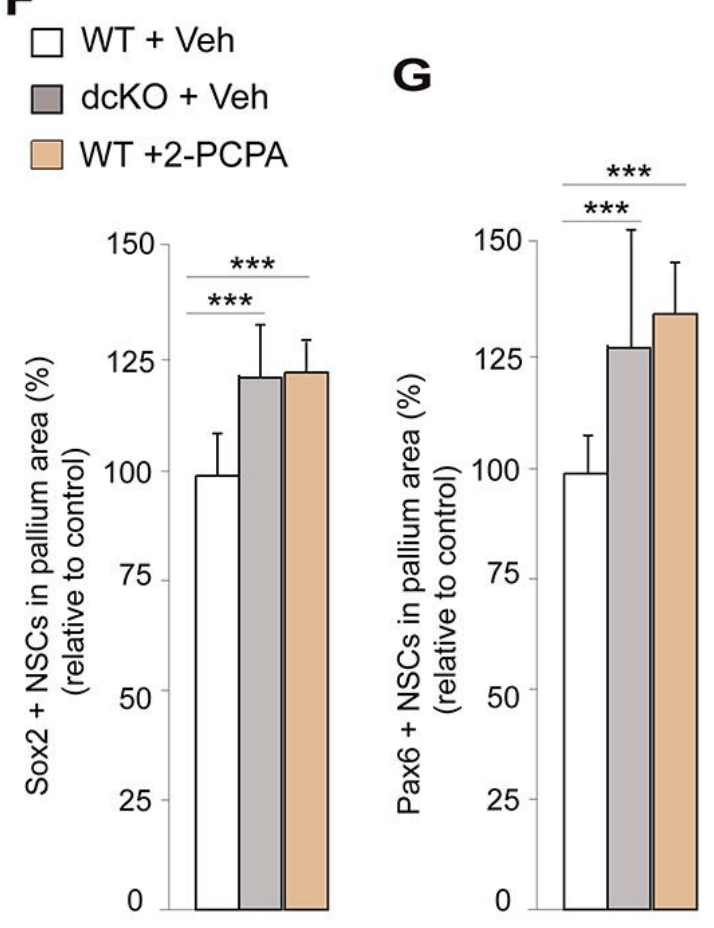
Figure S3.4 (related to figures 2, 4). Elevated level of H3K27me3 and H3K4me2 by inhibition of H3K27 and H3K4me2 demethylases caused the defect in neuronal differentiation in developing pallium. (A) Experimental paradigms in which WT embryos were treated with H3K27 demethylase inhibitor GSK-J4 and H3K4 demethylase inhibitor 2-PCPA. (B-G) IF $(B, E)$ and quantitative $(C, D, F, G)$ analyses are to compare cortical phenotype of Veh-treated WT, Veh-dcKO and WT which is treated with GSK-J4 (a-d) or with 2-PCPA (e-g). (C, D) Statistical comparisons indicate that the increased level of H3K27me3 in cortices of dcKO embryos or GSK-J4 treated embryos decreased the number of late-born Satb2 $^{+}(C)$ and Cux1 ${ }^{+}$neurons (D) compared to Veh-treated WT. Likewise, the increased level of H3K4me2 in cortices of dcKO embryos or 2-PCPA treated embryos increased the number of Sox2 ${ }^{+}(\mathrm{F})$ and Pax6+ NSCs (G) compared to Veh-treated WT. Values are expressed as means \pm SEMs $\left({ }^{\star} P<0.05 ;{ }^{\star \star} P<0.01 ;{ }^{* \star} P<0.005\right)$. Abbreviations: MP, medial pallium; $\mathrm{DP}$, dorsal pallium; LP, lateral pallium. Scale bars $=100 \mu \mathrm{m}(10 \mathrm{x}, \mathrm{B})$ and $50 \mu \mathrm{m}(40 \mathrm{x}, \mathrm{B})$. 
Chapter 3

A Control dcKO_hGFAP-Cre
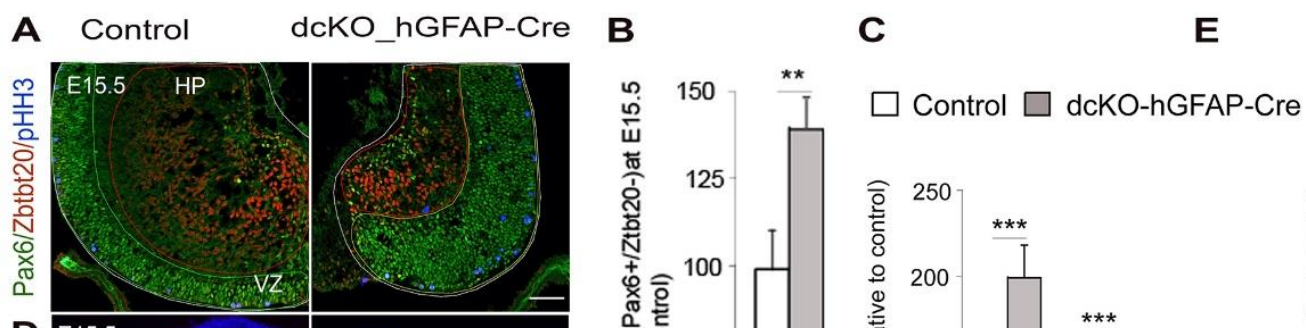

D E15.5
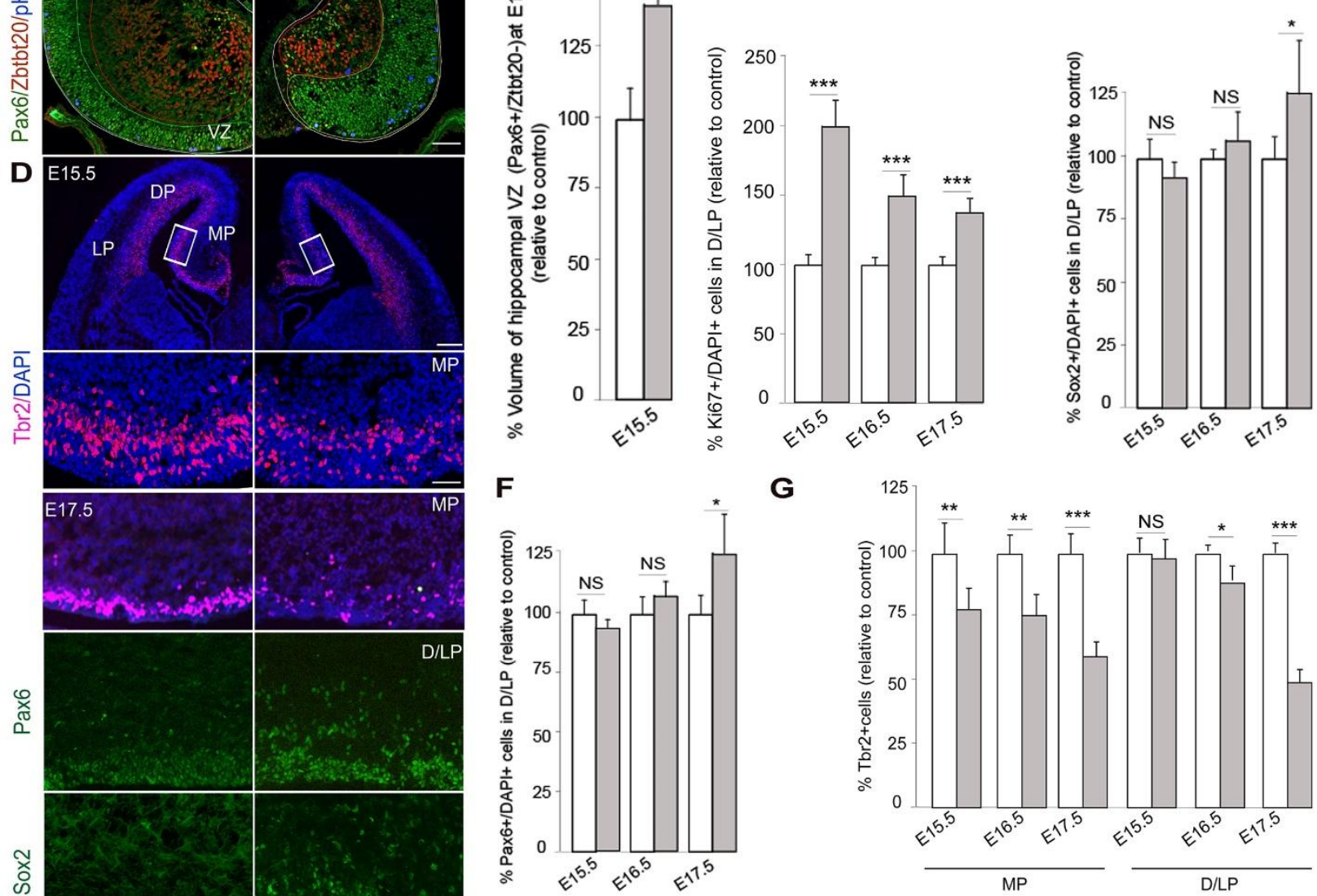

G
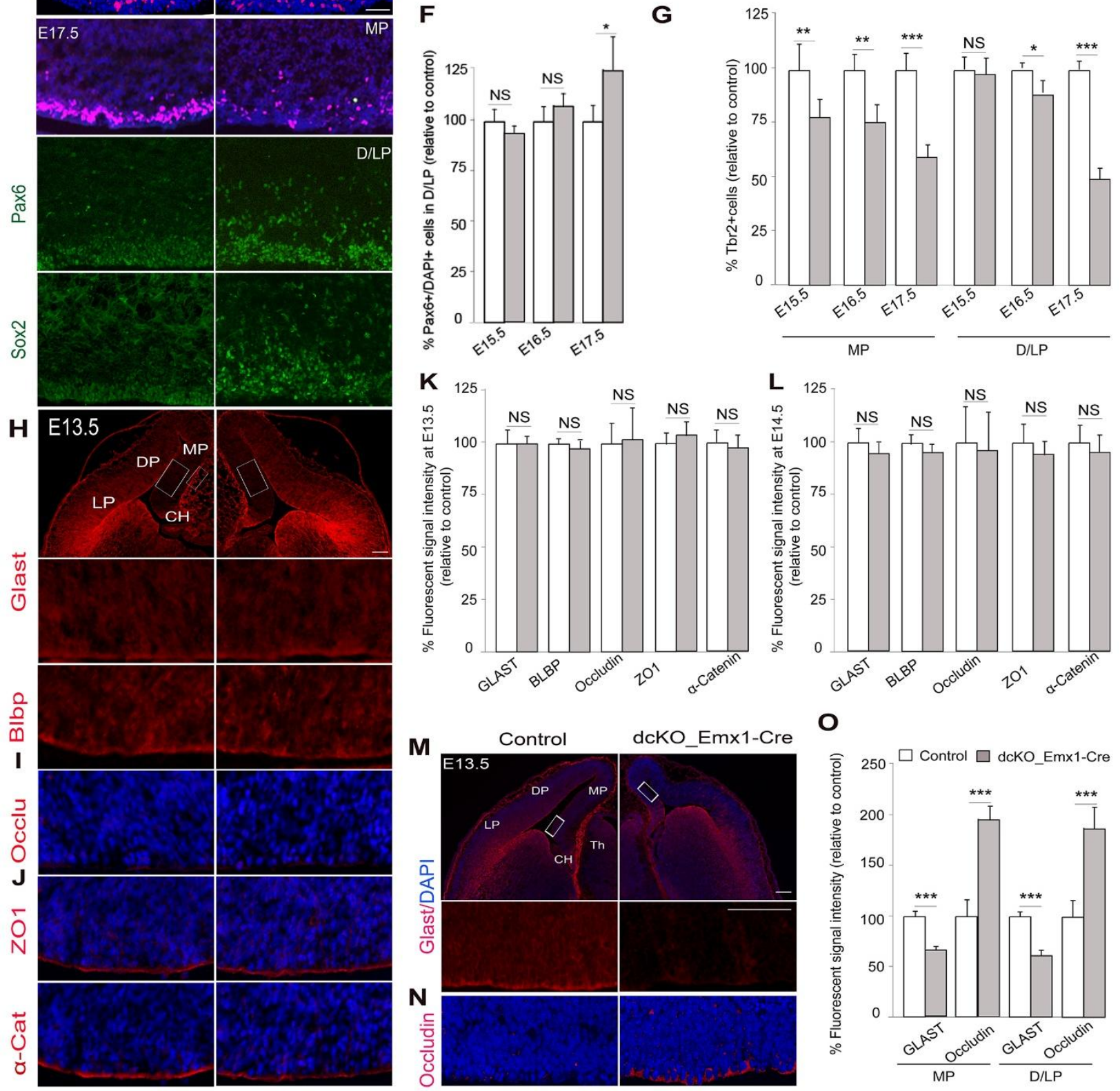
Figure S3.5 (related to figures 4, 5). Loss of BAF155 and BAF170 in dcKO mutants has a profound effect on the pool of NSCs, expression of adherens junction molecules.

(A) Representative images of triple IF analysis of Pax6 to visualize hippocampal VZ, Ztbt20 to mark hippocampal plate (HP) and pHH3 to label mitotic M-phase cells in our 3D reconstruction analysis. Total hippocampal volume was surrounded by white line. (B) Quantitative analyses of the volume of the hippocampal VZ (Pax6 $\left.{ }^{+} / \mathrm{Ztbt}^{-}\right)$in the dcKO_hGFAP-Cre mutant and control at E15.5 (see also Movie S1 for 3D reconstruction analysis). (C-G) IF (D) and quantitative (C, E, F and G) analyses of the mitotic marker Ki67 (C), RG markers Pax6 and Sox2 (D, E and F), and IP marker Tbr2 in the MP and D/LP of dcKO_hGFAP-Cre mutants and control (G). Generally, the loss of BAF155 and BAF170 in dcKO_hGFAP-Cre mutants had a more profound effect on the pool of NSCs in the MP (see also Fig. 4E-L) than in the D/LP. It should be noted that the loss of BAF155 and BAF170 in the E15.5-E16.5 D/LP did not affect the pool of $\mathrm{Pax6}^{+} / \mathrm{Sox} 2^{+} \mathrm{RGs}$; however, the number of $\mathrm{Ki} 67^{+}$active mitotic progenitors was already increased. Counting was done in selected frames, denoted by white boxes. $(\mathrm{H}-\mathrm{L})$ IF $(\mathrm{H}-\mathrm{J})$ and quantitative $(\mathrm{K}, \mathrm{L})$ analyses revealed that in constract to later stages (E15.5, E16.5), there is no obvious difference in expression level of GLAST, BLBP, ZO1, a-Catenin and Occludin between the control and dcKO_hGFAP-Cre pallium at E13.5 and E14.5. (M-O) Immunostaining of coronal sections from the control and dcKO_Emx1-Cre pallium at E13.5 for GLAST (M) and occludin (N) revealed an altered cell identity from GLAST $^{\text {high+}} /$ Occludin- RGs in controls to GLAST $^{\text {low+}} /$ occludin $^{\text {high+ }}$ NEs in the dcKO_Emx1-Cre mutant. (O) Quantitative analyses of panels $\mathrm{M}-\mathrm{N}$ are shown. Values are expressed as means \pm SEMs $\left({ }^{*} P<0.05 ;{ }^{* *} P<0.01 ;{ }^{* *} P<0.005\right)$. Abbreviations: MP, medial pallium; DP, dorsal pallium; LP, lateral pallium; HP, hippocampal plate; VZ, ventricular zone; $\mathrm{CH}$, cortical hem; Th, thalamus. Scale bar $=100 \mu \mathrm{m}$. 
A

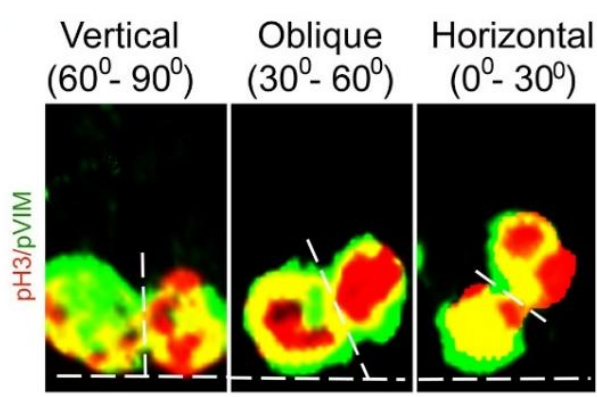

B

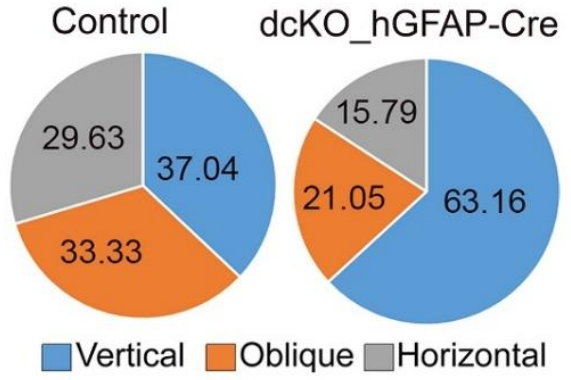

C

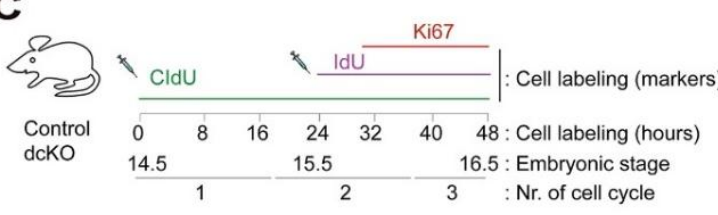

CldU+/ldU-/Ki67- : cells exit from 1st cell cycle

CldU+/ldU+/Ki67- : cells enter into 2 nd cell cycle and exit before 3 rd cycle.

$\mathrm{CldU}+/ / \mathrm{dU}+/ \mathrm{Ki} 67+$ : cells enter into 3 rd cell cycle.

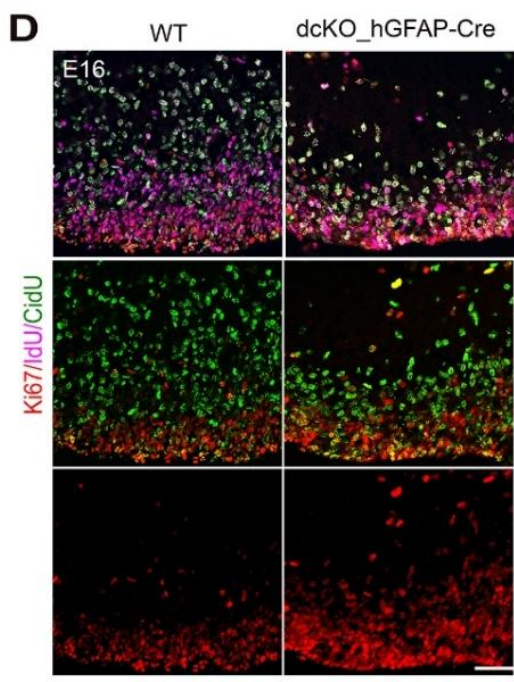

$\mathbf{F}$

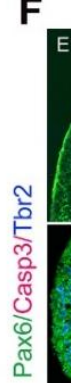

Control

dcKO hGFAP-Cre

L Control dckO Nex

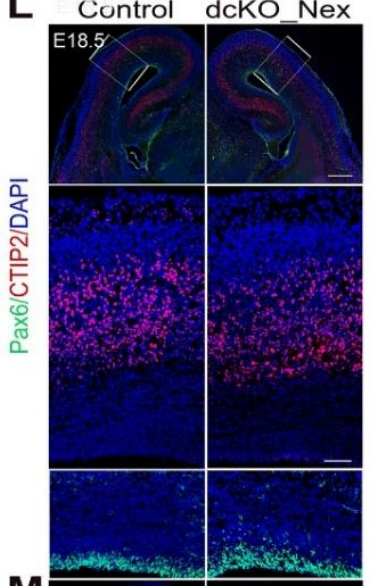

$\mathbf{M}$

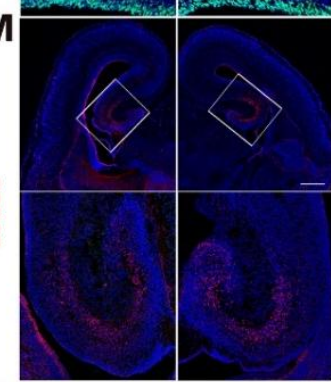

$\mathbf{N}$

$\square$ Control $\square$ dcKO_Nex-Cre

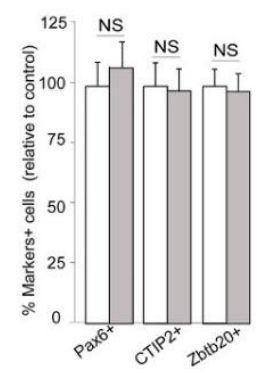

K

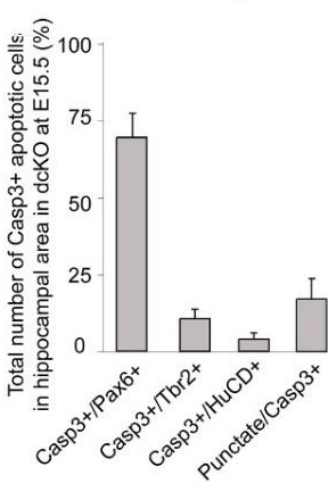


Figure S3.6 (related to figures 2-5). The spindle orientation, selective apoptosis of RGs in dcKO_hGFAP-Cre mutants and phenotypes of cortical neuron-specific dcKO_Nex-Cre mutants.

(A, B) IF analyses in control and dcKO mutants using antibodies against $\mathrm{pHH} 3$ and $\mathrm{pVIM}$ to visualize the orientation of spindles (vertical, oblique, horizontal) (A), and quantitative analyses (B) indicate that loss of BAF155 and BAF170 induces proliferative, symmetric division. (C) Experimental paradigm for determining cell-cycle parameters and color scheme for immunolabeling of micrographs (in D). (D) Images show triple-label IHC of cortical sections with antibodies for CidU (48-h labeling), IdU (24-h labeling) to mark both exited and cycling progenitors, Ki67 to label proliferating progenitors at E16.5. (E) Statistical comparisons indicated a decreased number of cells, which exit from $1^{\text {st }}$ and $2^{\text {nd }}$ cell cycles and an increased number of progenitors, which enter the 3rd cycle in the medial pallium of dcKO embryos compared with control. (F-K) Triple IF for Pax6/Tbr2/Casp3 and HuCD/TUBB3/Casp3 (F-I) and quantitative analyses $(\mathrm{J}, \mathrm{K})$ show that the developing hippocampus of the dcKO-hGFAP-Cre embryo has a high number of Casp3 $^{+}$apoptotic cells at E14.5-E18.5 (F-J). Notably, most Casp3+ cells are immunoreactive for Pax6 (white filled arrows) or exhibit punctate forms (late phase of apoptosis, empty arrows), whereas less extended cells are Tbr2 $^{+}$IPs and $\mathrm{HuCD}^{+} / \mathrm{TUBB}^{+}$neurons (F, H, K; arrowhead). Lower panels are higher-magnification images from the fields indicated by white rectangles. $(L-N)$ Phenotype analysis of the pallium from neuron-specific dcKO_Nex-Cre mutants. Immunostaining of coronal sections of E18.5 brains with antibodies specific for RGs (Pax6; L), layer 5 neurons (Ctip2; L) and hippocampal neurons (Ztbt20; M), and quantification of results in (L) showed no evident differences in the number of immunopositive cells between the dcKO_Nex-Cre and control pallium. Values are presented as means \pm SEMs $\left({ }^{*} P<0.05,{ }^{* *} P<0.01,{ }^{* *} P<0.005\right)$. Scale bars $=100 \mu \mathrm{m}(10 x ; F, H, L, M), 50 \mu \mathrm{m}$ (40x; F, G, H and I), $100 \mu \mathrm{m}$ (L) and $50 \mu \mathrm{m}$ (D). 
Chapter 3

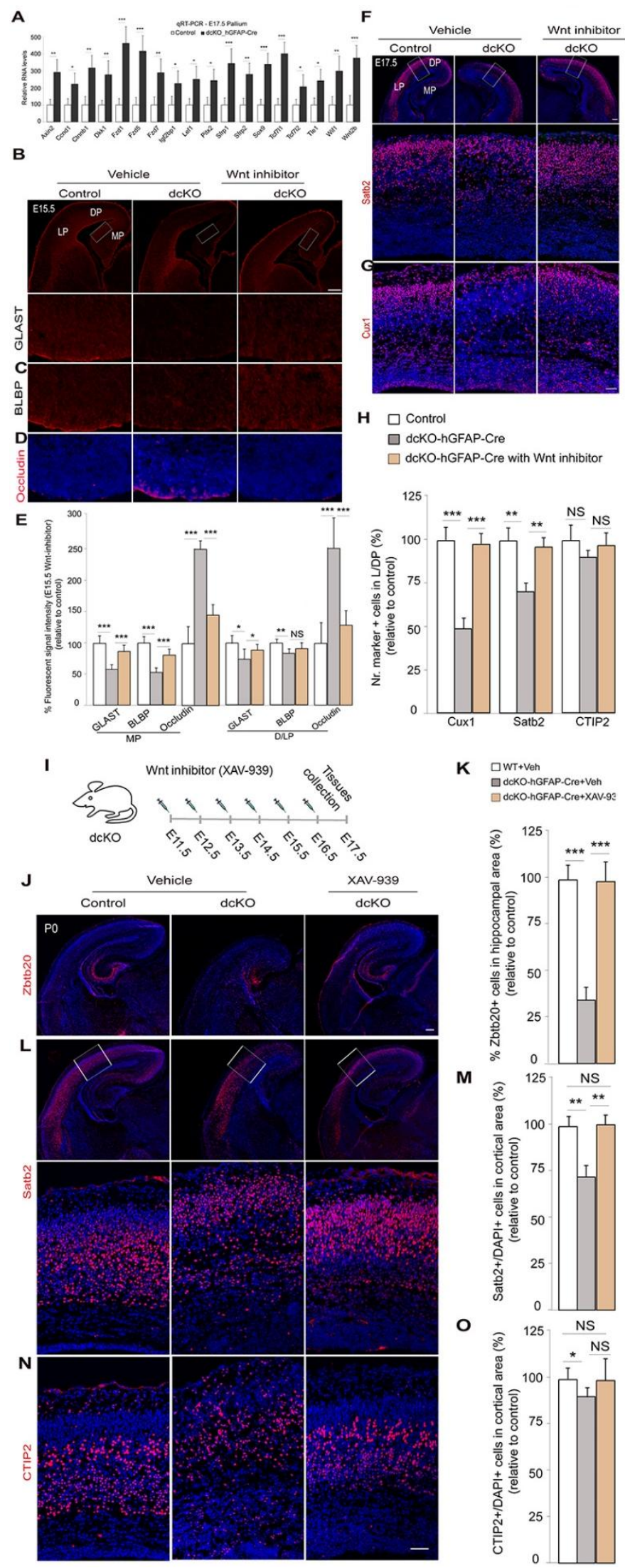


Figure S3.7 (related to figures 6,7 ). Suppression of Wnt signaling in the developing pallium by BAF complexes in the developing forebrain.

(A) Expression of upregulated Wnt genes identified by RNA-Seq analysis (see Fig. 6A, B) was further verified by qRT-PCR. (B-E) IF (B-D) and quantitative (E) analyses of dcKO_hGFAP-Cre mutants at E15.5 showing the effects of treatment with the Wnt inhibitor ICG-001 on expression of the RG markers GLAST (B) and BLBP (C), and the NE marker occludin (D). IF (F-G) and quantitative (H) analyses of dcKO_hGFAP-Cre mutants at E17.5, showing the effects of treatment with WNTi on pools of Satb2+ $(F), \mathrm{Cux1}^{+}(\mathrm{G})$ cortical neurons in L/DP. Quantitative analyses indicated that inhibition of Wnt signaling largely rescues defects in L/DP in dcKO_hGFAP-Cre mutants $(\mathrm{H})$. $(\mathrm{I}-\mathrm{O})$ Rescued cortical defects in dcKO_hGFAP-Cre mutant by Wnt inhibitor XAV-939. (I) Experimental paradigm in which WT (control), dcKO embryos were treated with Wht inhibitor XAV-939 or Vehicle (Veh). (J-O) IF $(\mathrm{J}, \mathrm{L}, \mathrm{N})$ and quantitative $(\mathrm{K}, \mathrm{M}, \mathrm{O})$ analyses are to compare population of cortical neuron: Ztbt20+ hippocampal neurons $(\mathrm{J}, \mathrm{K})$, Satb2+ late-born neurons $(\mathrm{L}, \mathrm{M})$, Ctip2+ early-born neurons $(\mathrm{N}, \mathrm{O})$ in Veh-treated WT, Veh-treated dcKO and WNTi-treated WT. Quantitative analyses indicated that inhibition of Wnt signaling by XAV-939 largely rescues the neurogenesis defects in dcKO_hGFAP-Cre mutants. Values are expressed as means \pm SEMs $\left({ }^{*} P<0.05 ;{ }^{* \star} P<0.01 ;{ }^{* *} P<0.005 ; \mathrm{n}=4\right)$. Abbreviations: MP, medial pallium; DP, dorsal pallium; LP, lateral pallium. Scale bars = $100 \mu \mathrm{m}(10 \mathrm{x} ; \mathrm{B}, \mathrm{F}), 50 \mu \mathrm{m}(40 \mathrm{x} ; \mathrm{G}), 100 \mu \mathrm{m}$ $(10 x ; J)$, and $50 \mu \mathrm{m}(40 x ; N)$. 


\section{Chapter 4: General discussion}

Previous studies have reported the requirement of the BAF complex in neural development and maintenance of normal nervous system function. The BAF complex was identified in such studies to play critical roles in altering chromatin conformation to regulate gene expression programs that underline neurodevelopmental processes, including specification of brain structures, and neurogenesis (Ho et al., 2011; Narayanan et al., 2015a; Bachmann et al., 2016b; Nguyen et al., 2016; Kadoch et al., 2017; Nguyen et al., 2018).

The findings presented in this thesis show that the chromatin remodeler BAF regulate embryonic and forebrain development. The BAF complex controls proliferation and differentiation of neural stem/progenitor cells during early and late embryonic development through modulation of discrete epigenetic mechanisms. We previously showed that during corticogenesis, BAF complex modulates activity of H3K27 demethylases to influence global distribution of the heterochromatin marks H3K27me2/3 (Narayanan et al., 2015a).

In the current study, increased levels of $\mathrm{H} 3 \mathrm{~K} 27 \mathrm{me} 2 / 3$ repressive marks due to loss of BAF complex were observed in the entire mouse embryo or specifically in the early and late developing forebrain (Tran et al., 2013; Bachmann et al., 2016b; Nguyen et al., 2016; Tran 2017; Nguyen et al., 2018). Normally, the BAF complex is known to antagonize the polycomb repressor complex known to deposit H3K27me2/3 repressive marks on target histones leading to chromatin condensation and inactivation (Ho et al., 2011; Kadoch et al., 2017). Indeed, in the absence of the H3K27 methyltransferase EZH2, H3K27me3 repressive marks are diminished, causing cortical neural progenitors in the developing forebrain to adopt neurogenic differentiative division mode (Pereira et al., 2010). By inhibiting histone tagging by 
$\mathrm{H} 3 \mathrm{~K} 27 \mathrm{me2} / 3$, the chromatin remodeling BAF complex is able to cause cell cycle exit of progenitor cells and their further differentiation during early cortical development. The phenotype of the malformed dcKO_Foxg1-Cre forebrain and dcKO_CAG-Cre embryo is thus reflective of perturbation of the epigenetic balance in the installation and removal of the heterochromatin marks $\mathrm{H} 3 \mathrm{~K} 27 \mathrm{me} 2 / 3$ that is necessary for proper embryonic development, including early cortical development.

By probing the mechanistic details of how BAF complex regulates late cortical development, we were able to determine in the second study that BAF complex targets neural proliferation and differentiation genes and remodels their epigenetic environment to drive proper corticogenesis. The dcKO_hGFAP-Cre model used in the study (Nguyen et al., 2016) allowed investigation of late cortical developmental events to complement the findings obtained in the early developing dcKO_Foxg1-Cre forebrain (Nguyen et al., 2016). Apart from regulating dynamics of the repressive mark $\mathrm{H} 3 \mathrm{~K} 27 \mathrm{me} 2 / 3$, it was determined that the BAF complex concurrently dictates establishment of the transcription activation mark H3K4me2 to influence the progression of late cortical development. Of note, inactivation of BAF complex due to genetic ablation of its scaffolding subunit BAF155 and BAF170 caused H3K27me3-mediated silencing of genes involved in neuronal differentiation and concurrently promoted unrestricted proliferation of neural stem cells via enhancement of H3K4me2 mark at related gene loci. In the entire process of neural (brain) development, relevant genes are enriched with $\mathrm{H} 3 \mathrm{~K} 4 \mathrm{me} 2$ at the expense of H3K27me2/3 to favor transcriptional activity and instruct various neural cell developmental processes. As an indication of it importance, $\mathrm{H} 3 \mathrm{~K} 4 \mathrm{me} 2$ appear early in neural stem cells and progressively gains prominence in the course brain development (Zhang et al., 2012). However, Hahn and colleagues found that the H3K27me3 marks at intergenic regions decrease during differentiation of neural progenitors 
(Hahn et al., 2013). In part, this explains why the increase in H3K27me3 following loss of BAF complex negatively impacted neural cell cycle kinetics leading to disturbance of cortical development.

The upregulated Wnt signaling in the dcKO_hGFAP-Cre cortex and subsequent restoration of normal cortical and hippocampal phenotypes through Wnt inhibition indicate that the BAF complex is a potential suppressor of the Wnt signaling pathway during development of the cerebral cortex. This finding is partly in agreement with previous studies that found that the BAF complex can either activate or repress Wnt signaling activity (Barker et al., 2001a; Ronan et al., 2013; Vasileiou et al., 2015).

Put together, BAF155 and BAF170 are essential in maintaining the stability and function of the BAF complex during organogenesis. In the event of the ablation of BAF155 and BAF170, there is loss of function of the BAF complex which leads to impairment of brain development via abnormal transcriptional and epigenetic modalities, including $\mathrm{H} 3 \mathrm{~K} 27 \mathrm{me} 3$ and $\mathrm{H} 3 \mathrm{~K} 4 \mathrm{me} 2$ imbalance and Wnt signaling dysregulation. 


\section{Summary}

The multi-subunit BAF (SWI/SNF) complex is capable of using energy generated from ATP hydrolysis to reorder chromatin structure. Such chromatin changes are known to affect cell biological processes through gene expression regulation. Thus, BAF complex plays pivotal roles in many developmental events. As a focus of the studies present, we identified the BAF complex as a powerful regulator of coticogenesis and embryogenesis. In this study, we employed a novel mouse model system in which deletion of the BAF complex subunits BAF155 and BAF170 results in proteomic degradations of the entire BAF complex and resultant loss of its epigenetic function. The first part of the thesis, highlights how the BAF complex stability is dependent on the dual presence of the scaffolding subunits BAF155 and BAF170, and the disturbance in the H3K27me2/3 epigenetic landscape when they are ablated specifically in the forebrain under the control Foxg1-Cre activity or in the entire embryo using the ubiquitous-inducible CAG-Cre. Preliminary evidence of the involvement of BAF complex in cortical development was thus obtained in the first part of the studies. In the second part, we gathered conclusive evidence on how the BAF complex regulates cortical and hippocampal development. Our investigation in the dcKO_hGFAP_Cre developing cortex revealed the BAF complexes induces heterochromatin state at gene loci involved in neural progenitor proliferation and Wnt-signaling; leading to their suppression. On the other hand, the BAF complex enhances the transcription of neuronal differentiation-related genes by promoting euchromatin formation at associated genomic regions. Together, we reported that the activity of the BAF complex ensures the appropriate proliferative capacity of neural progenitors and their neuronal output in late cortical development. 


\section{References}

Aaku-Saraste E, Hellwig A, Huttner WB. 1996. Loss of occludin and functional tight junctions, but not ZO-1, during neural tube closure--remodeling of the neuroepithelium prior to neurogenesis. Developmental Biology 180(2): 664-679.

Agger K, Cloos PA, Christensen J, Pasini D, Rose S, Rappsilber J, Issaeva I, Canaani E, Salcini AE, Helin K. 2007. UTX and JMJD3 are histone H3K27 demethylases involved in HOX gene regulation and development. Nature 449(7163): 731-734.

Agger K, Cloos PA, Rudkjaer L, Williams K, Andersen G, Christensen J, Helin K. 2009. The H3K27me3 demethylase JMJD3 contributes to the activation of the INK4A-ARF locus in response to oncogene- and stress-induced senescence. Genes \& development 23(10): 1171-1176.

Albert M, Kalebic N, Florio M, Lakshmanaperumal N, Haffner C, Brandl H, Henry I, Huttner WB. 2017. Epigenome profiling and editing of neocortical progenitor cells during development. The EMBO journa/36(17): 2642-2658.

Attanasio C, Nord AS, Zhu Y, Blow MJ, Biddie SC, Mendenhall EM, Dixon J, Wright C, Hosseini R, Akiyama JA, et al. 2014. Tissue-specific SMARCA4 binding at active and repressed regulatory elements during embryogenesis. Genome Res 24(6): 920-929.

Bachmann C, Nguyen H, Rosenbusch J, Pham L, Rabe T, Patwa M, Sokpor G, Seong RH, Ashery-Padan R, Mansouri A, et al. 2016a. mSWI/SNF (BAF) Complexes Are Indispensable for the Neurogenesis and Development of Embryonic Olfactory Epithelium. PLOS Genetics 12(9): e1006274-e1006274.

Bailey C, Soane E, Delbridge R, Alfes K, Shantz A, Petrov G. 2011. Employee engagement, organisational performance and individual well-being: exploring the evidence, developing the theory. The International Journal of Human Resource Management 22(1):232-233 
Barker N, Hurlstone A, Musisi H, Miles A, Bienz M, Clevers H. 2001. The chromatin remodelling factor Brg-1 interacts with beta-catenin to promote target gene activation. The EMBO journal 20(17): 4935-4943.

Bernstein BE, Meissner A, Lander ES. 2007. The Mammalian Epigenome. Cell 128(4): 669-681.

Bird A. 2007. Perceptions of epigenetics. Nature 447: 396.

Bohmdorfer G, Wierzbicki AT. 2015. Control of Chromatin Structure by Long Noncoding RNA. Trends in cell biology 25(10): 623-632.

Boland MJ, Nazor KL, Loring JF. 2014. Epigenetic regulation of pluripotency and differentiation. Circulation research 115(2): 311-324.

Cao R, Wang L, Wang H, Xia L, Erdjument-Bromage H, Tempst P, Jones RS, Zhang Y. 2002. Role of histone H3 lysine 27 methylation in Polycomb-group silencing. Science 298(5595): 1039-1043.

Clapier CR, Iwasa J, Cairns BR, Peterson CL. 2017. Mechanisms of action and regulation of ATP-dependent chromatin-remodelling complexes. Nature Reviews Molecular Cell Biology 18(7):407-422.

Conti L, Pollard SM, Gorba T, Reitano E, Toselli M, Biella G, Sun Y, Sanzone S, Ying QL, Cattaneo E, et al. 2005. Niche-independent symmetrical self-renewal of a mammalian tissue stem cell. PLoS Bio/ 3(9): e283.

Coskun V, Tsoa R, Sun YE. 2012. Epigenetic regulation of stem cells differentiating along the neural lineage. Current Opinion in Neurobiology 22(5): 762-767.

Chen J, Bardes EE, Aronow BJ, Jegga AG. 2009. ToppGene Suite for gene list enrichment analysis and candidate gene prioritization. Nucleic Acids Res 37(Web Server issue): W305-311.

Chenn A, Walsh CA. 2002. Regulation of cerebral cortical size by control of cell cycle exit in neural precursors. Science 297(5580): 365-369. 
Choi J, Ko M, Jeon S, Jeon Y, Park K, Lee C, Lee H, Seong RH. 2012. The SWI/SNF-like BAF complex is essential for early B cell development. Journal of immunology 188(8): 3791-3803.

De Santa F, Totaro MG, Prosperini E, Notarbartolo S, Testa G, Natoli G. 2007. The histone H3 lysine-27 demethylase Jmjd3 links inflammation to inhibition of polycomb-mediated gene silencing. Cell 130(6): 1083-1094.

Dehay C, Kennedy H. 2007. Cell-cycle control and cortical development. Nat Rev Neurosci 8(6): 438-450.

Djebali S, Davis CA, Merkel A, Dobin A, Lassmann T, Mortazavi A, Tanzer A, Lagarde J, Lin W, Schlesinger F. 2012. Landscape of transcription in human cells. Nature 489(7414): 101-108.

Draganova K, Zemke M, Zurkirchen L, Valenta T, Cantu C, Okoniewski M, Schmid MT, Hoffmans R, Gotz M, Basler K, et al. 2015. Wnt/beta-catenin signaling regulates sequential fate decisions of murine cortical precursor cells. Stem Cells 33(1): 170-182.

Durak O, Gao F, Kaeser-Woo YJ, Rueda R, Martorell AJ, Nott A, Liu CY, Watson LA, Tsai LH. 2016. Chd8 mediates cortical neurogenesis via transcriptional regulation of cell cycle and Wnt signaling. Nature Neuroscience 19(11): 1477-1488.

Schmechel D, Rakic P. 1979. A golgi study of radial glial cells in developing monkey telencephalon: Morphogenesis and transformation into astrocytes. Anatomy and Embryology 156(2):115-52

Elsen GE, Bedogni F, Hodge RD, Bammler TK, MacDonald JW, Lindtner S, Rubenstein JLR, Hevner RF. 2018. The Epigenetic Factor Landscape of Developing Neocortex Is Regulated by Transcription Factors Pax6 $\rightarrow$ Tbr2 $\rightarrow$ Tbr1. Front Neurosci 12(571).

Erhardt S, Su IH, Schneider R, Barton S, Bannister AJ, Perez-Burgos L, Jenuwein T, Kouzarides T, Tarakhovsky A, Surani MA. 2003. Consequences of the 
depletion of zygotic and embryonic enhancer of zeste 2 during preimplantation mouse development. Development 130(18): 4235-4248.

Feng J, Liu T, Qin B, Zhang Y, Liu XS. 2012. Identifying ChIP-seq enrichment using MACS. Nature protocols 7(9): 1728-1740.

Flaus A, Martin DMA, Barton GJ, Owen-Hughes T. 2006. Identification of multiple distinct Snf2 subfamilies with conserved structural motifs. Nucleic acids research 34(10): 2887-2905.

Florio M, Huttner WB. 2014. Neural progenitors, neurogenesis and the evolution of the neocortex. Development 141(11): 2182.

Gao P, Postiglione MP, Krieger TG, Hernandez L, Wang C, Han Z, Streicher C, Papusheva E, Insolera R, Chugh K, et al. 2014. Deterministic progenitor behavior and unitary production of neurons in the neocortex. Cell 159(4): 775-788.

Goebbels S, Bormuth I, Bode U, Hermanson O, Schwab MH, Nave KA. 2006. Genetic targeting of principal neurons in neocortex and hippocampus of NEXCre mice. Genesis 44(12): 611-621.

Goldberg AD, Allis CD, Bernstein E. 2007. Epigenetics: A Landscape Takes Shape. Cell 128(4): 635-638.

Gorski JA, Talley T, Qiu M, Puelles L, Rubenstein JL, Jones KR. 2002. Cortical excitatory neurons and glia, but not GABAergic neurons, are produced in the Emx1-expressing lineage. The Journal of neuroscience : the official journal of the Society for Neuroscience 22(15): 6309-6314.

Gotz M, Huttner WB. 2005. The cell biology of neurogenesis. Nat Rev Mol Cell Bio/6(10): 777-788.

Griffin CT, Curtis CD, Davis RB, Muthukumar V, Magnuson T. 2011. The chromatin-remodeling enzyme BRG1 modulates vascular Wnt signaling at two 
levels. Proceedings of the National Academy of Sciences of the United States of America 108(6): 2282-2287.

Gutiérrez J, Paredes R, Cruzat F, A Hill D, Wijnen A, Lian J, Stein G, L Stein J, Imbalzano A, A. Montecino M. 2007. Chromatin Remodeling by SWI/SNF Results in Nucleosome Mobilization to Preferential Positions in the Rat Osteocalcin Gene Promoter. J Biol Chem 282(13): 9445-57.

Hahn MA, Qiu R, Wu X, Li AX, Zhang H, Wang J, Jui J, Jin SG, Jiang Y, Pfeifer GP, et al. 2013. Dynamics of 5-hydroxymethylcytosine and chromatin marks in Mammalian neurogenesis. Cell Rep 3(2): 291-300.

Halder R, Hennion M, Vidal RO, Shomroni O, Rahman R-U, Rajput A, Centeno TP, van Bebber F, Capece V, Vizcaino JCG. 2016. DNA methylation changes in plasticity genes accompany the formation and maintenance of memory. Nature neuroscience 19(1): 102-110.

Hanna CW, Demond H, Kelsey G. 2018. Epigenetic regulation in development: is the mouse a good model for the human? Human reproduction update 24(5): 556-576.

Hartfuss E, Galli R, Heins N, Götz M. 2001. Characterization of CNS Precursor Subtypes and Radial Glia. Developmental Biology 229(1): 15-30.

Hayashi S, McMahon AP. 2002. Efficient recombination in diverse tissues by a tamoxifen-inducible form of Cre: a tool for temporally regulated gene activation/inactivation in the mouse. Dev Biol 244(2): 305-318.

Heard E, Martienssen RA. 2014. Transgenerational epigenetic inheritance: myths and mechanisms. Cell 157(1): 95-109.

Hebert JM, McConnell SK. 2000. Targeting of cre to the Foxg1 (BF-1) locus mediates loxP recombination in the telencephalon and other developing head structures. Dev Biol 222(2): 296-306. 
Heinz S, Benner C, Spann N, Bertolino E, Lin YC, Laslo P, Cheng JX, Murre C, Singh H, Glass CK. 2010. Simple combinations of lineage-determining transcription factors prime cis-regulatory elements required for macrophage and B cell identities. Molecular cell 38(4): 576-589.

Helming KC, Wang X, Roberts CW. 2014. Vulnerabilities of mutant SWI/SNF complexes in cancer. Cancer Cell 26(3): 309-317.

Hirabayashi Y, Gotoh Y. 2010. Epigenetic control of neural precursor cell fate during development. Nature reviews. Neuroscience 11(6): 377-388.

Hirabayashi Y, Suzki N, Tsuboi M, Endo T, Toyoda T, Shinga J, Koseki H, Vidal M, Gotoh Y. 2009. Polycomb Limits the Neurogenic Competence of Neural Precursor Cells to Promote Astrogenic Fate Transition. Neuron 63(5): 600-13.

Hirschhorn JN, Brown SA, Clark CD, Winston F. 1992. Evidence that SNF2/SWI2 and SNF5 activate transcription in yeast by altering chromatin structure. Genes \& development 6(12A): 2288-2298.

Ho L, Crabtree GR. 2011. Chromatin remodelling during development. Nature 463(7280): 474-484.

Ho L, Miller EL, Ronan JL, Ho WQ, Jothi R, Crabtree GR. 2011. esBAF facilitates pluripotency by conditioning the genome for LIF/STAT3 signalling and by regulating polycomb function. Nature Cell Biology 13(8): 903-913.

Ho L, Ronan JL, Wu J, Staahl BT, Chen L, Kuo A, Lessard J, Nesvizhskii Al, Ranish J, Crabtree GR. 2009a. An embryonic stem cell chromatin remodeling complex, esBAF, is essential for embryonic stem cell self-renewal and pluripotency. Proc Natl Acad Sci U S A 106(13): 5181-5186.

Hong S, Cho YW, Yu LR, Yu H, Veenstra TD, Ge K. 2007. Identification of JmjC domain-containing UTX and JMJD3 as histone H3 lysine 27 demethylases. Proceedings of the National Academy of Sciences of the United States of America 104(47): 18439-18444. 
Jepsen K, Solum D, Zhou T, McEvilly RJ, Kim HJ, Glass CK, Hermanson O, Rosenfeld MG. 2007. SMRT-mediated repression of an H3K27 demethylase in progression from neural stem cell to neuron. Nature 450(7168): 415-419.

Juliandi B, Abematsu M, Nakashima K. 2010. Chromatin remodeling in neural stem cell differentiation. Current Opinion in Neurobiology 20(4): 408-415.

Kadoch C, Copeland RA, Keilhack H. 2016. PRC2 and SWI/SNF Chromatin Remodeling Complexes in Health and Disease. Biochemistry 55(11): 1600-14.

Kadoch C, Hargreaves DC, Hodges C, Elias L, Ho L, Ranish J, Crabtree GR. 2013. Proteomic and bioinformatic analysis of mammalian SWI/SNF complexes identifies extensive roles in human malignancy. Nature genetics 45(6): 592-601.

Kadoch C, Williams RT, Calarco JP, Miller EL, Weber CM, Braun SMG, Pulice JL, Chory EJ, Crabtree GR. 2017. Dynamics of BAF-Polycomb complex opposition on heterochromatin in normal and oncogenic states. Nature genetics 49(2): 213-222.

Ko M, Sohn DH, Chung H, Seong RH. 2008. Chromatin remodeling, development and disease. Mutation research 647(1-2): 59-67.

Kojima Y, Tam OH, Tam PP. 2014. Timing of developmental events in the early mouse embryo. Seminars in cell \& developmental biology 34: 65-75.

Kouzarides T. 2007. Chromatin modifications and their function. Cell 128(4): 693-705.

Kriegstein A, Alvarez-Buylla A. 2009. The glial nature of embryonic and adult neural stem cells. Annual review of neuroscience 32: 149-184.

Kruidenier L, Chung CW, Cheng Z, Liddle J, Che K, Joberty G, Bantscheff M, Bountra C, Bridges A, Diallo $H$, et al. 2012. A selective jumonji H3K27 demethylase inhibitor modulates the proinflammatory macrophage response. Nature 488(7411): 404-408. 
Lan F, Bayliss PE, Rinn JL, Whetstine JR, Wang JK, Chen S, Iwase S, Alpatov R, Issaeva I, Canaani E, et al. 2007. A histone H3 lysine 27 demethylase regulates animal posterior development. Nature 449(7163): 689-694.

Laurent BC, Treich I, Carlson M. 1993. The yeast SNF2/SWI2 protein has DNAstimulated ATPase activity required for transcriptional activation. Genes \& development 7(4): 583-591.

Lee MG, Villa R, Trojer P, Norman J, Yan KP, Reinberg D, Di Croce L, Shiekhattar R. 2007. Demethylation of H3K27 regulates polycomb recruitment and H2A ubiquitination. Science 318(5849): 447-450.

Lessard J, Wu Jl, Ranish JA, Wan M, Winslow MM, Staahl BT, Wu H, Aebersold R, Graef IA, Crabtree GR. 2007. An essential switch in subunit composition of a chromatin remodeling complex during neural development. Neuron 55(2): 201-215.

Li H, Handsaker B, Wysoker A, Fennell T, Ruan J, Homer N, Marth G, Abecasis G, Durbin R. 2009. The sequence alignment/map format and SAMtools. Bioinformatics 25(16): 2078-2079.

Lienhard M, Grimm C, Morkel M, Herwig R, Chavez L. 2014. MEDIPS: genomewide differential coverage analysis of sequencing data derived from DNA enrichment experiments. Bioinformatics 30(2): 284-286.

Love MI, Huber W, Anders S. 2014. Moderated estimation of fold change and dispersion for RNA-seq data with DESeq2. Genome biology 15(12): 555.

Machon O, Backman M, Machonova O, Kozmik Z, Vacik T, Andersen L, Krauss S. 2007. A dynamic gradient of Wnt signaling controls initiation of neurogenesis in the mammalian cortex and cellular specification in the hippocampus. Developmental Biology 311(1): 223-237.

Madisen L, Zwingman TA, Sunkin SM, Oh SW, Zariwala HA, Gu H, Ng LL, Palmiter RD, Hawrylycz MJ, Jones AR, et al. 2010. A robust and high-throughput Cre 
reporting and characterization system for the whole mouse brain. Nature Neuroscience 13(1): 133-140.

Malatesta P, Hack MA, Hartfuss E, Kettenmann H, Klinkert W, Kirchhoff F, Götz M. 2003. Neuronal or Glial Progeny: Regional Differences in Radial Glia Fate. Neuron 37(5): 751-764.

Mao Y, Ge X, Frank CL, Madison JM, Koehler AN, Doud MK, Tassa C, Berry EM, Soda T, Singh KK, et al. 2009. Disrupted in schizophrenia 1 regulates neuronal progenitor proliferation via modulation of GSK3beta/beta-catenin signaling. Cell 136(6): 1017-1031.

Martínez-Cerdeño V, Noctor SC, Kriegstein AR. 2006. The Role of Intermediate Progenitor Cells in the Evolutionary Expansion of the Cerebral Cortex. Cerebral Cortex 16(suppl_1): i152-i161.

Martynoga B, Drechsel D, Guillemot F. 2012. Molecular control of neurogenesis: a view from the mammalian cerebral cortex. Cold Spring Harbor perspectives in biology $\mathbf{4}(10)$.

Masliah-Planchon J, Bieche I, Guinebretiere JM, Bourdeaut F, Delattre O. 2015. SWI/SNF chromatin remodeling and human malignancies. Annual review of pathology 10: 145-171.

Mollgøard K, Saunders N. 1975. Complex tight junctions of epithelial and of endothelial cells in early foetal brain. $J$ Neurocytol 4(4):453-68.

Morest DK. 1970. A study of neurogenesis in the forebrain of opossum pouch young. Zeitschrift für Anatomie und Entwicklungsgeschichte 130(4): 265-305.

MuhChyi C, Juliandi B, Matsuda T, Nakashima K. 2013. Epigenetic regulation of neural stem cell fate during corticogenesis. International journal of developmental neuroscience : the official journal of the International Society for Developmental Neuroscience 31(6): 424-433. 
Mutch CA, Schulte JD, Olson E, Chenn A. 2010. Beta-catenin signaling negatively regulates intermediate progenitor population numbers in the developing cortex. PLoS One 5(8): e12376.

Narayanan R, Pirouz M, Kerimoglu C, Pham L, Wagener RJ, Kiszka KA, Rosenbusch J, Seong RH, Kessel M, Fischer A, et al. 2015. Loss of BAF (mSWI/SNF) Complexes Causes Global Transcriptional and Chromatin State Changes in Forebrain Development. Cell reports 13(9): 1842-1854.

Narayanan R, Pham L, Kerimoglu C, Watanabe T, Kiszka KA, Rosenbusch J, Seong RH, Fischer A, Stoykova A, Staiger JF, et al. Chromatin remodeling BAF155 subunit regulates the genesis of basal progenitors in developing cortex. iScience 4:109-126

Narayanan R, Tran. 2014. Roles of chromatin remodeling BAF complex in neural differentiation and reprogramming. Cell and Tissue Research 356(3): 575-584.

Narlikar GJ, Sundaramoorthy R, Owen-Hughes T. 2013. Mechanisms and functions of ATP-dependent chromatin-remodeling enzymes. Cell 154(3): 490-503.

Neigeborn L, Carlson M. 1984. Genes affecting the regulation of SUC2 gene expression by glucose repression in Saccharomyces cerevisiae. Genetics 108(4): 845-858.

Neilson C, Santos-Rosa H, Bannister A, Kouzarides T. 2006. Chromatin modifications and their function.

Nicol JW, Helt GA, Blanchard SG, Raja A, Loraine AE. 2009. The Integrated Genome Browser: free software for distribution and exploration of genomescale datasets. Bioinformatics 25(20): 2730-2731.

Nguyen H, Kerimoglu C, Pirouz M, Pham L, Kiszka KA, Sokpor G, Sakib MS, Rosenbusch J, Teichmann U, Seong RH, et al. 2018. Epigenetic Regulation by BAF Complexes Limits Neural Stem Cell Proliferation by Suppressing Wnt Signaling in Late Embryonic Development. Stem cell reports 10(6): 1734-1750. 
Nguyen H, Sokpor G, Pham L, Rosenbusch J, Stoykova A, Staiger JF, Tran. 2016. Epigenetic regulation by BAF ( $\mathrm{mSWI} / \mathrm{SNF}$ ) chromatin remodeling complexes is indispensable for embryonic development. Cell cycle 15(10): 1317-1324.

Misson J, P. Austin C, Takahashi T, L. Cepko C, S. Caviness V. 1991. The Alignment of Migrating Neural Cells in Relation to the Murine Neopallial Radial Glial Fiber System. Cereb Cortex 1(3): 221-9.

Pedersen MT, Helin K. 2010. Histone demethylases in development and disease. Trends in cell biology 20(11): 662-671.

Pereira HM, Leadley PW, Proença V, Alkemade R, Scharlemann JPW, FernandezManjarrés JF, Araújo MB, Balvanera P, Biggs R, Cheung WWL, et al. 2010. Scenarios for Global Biodiversity in the 21st Century. Science 330(6010): 1496-1501.

Pereira JD, Sansom SN, Smith J, Dobenecker MW, Tarakhovsky A, Livesey FJ. 2010. Ezh2, the histone methyltransferase of $\mathrm{PRC2}$, regulates the balance between self-renewal and differentiation in the cerebral cortex. Proceedings of the National Academy of Sciences of the United States of America 107(36): 15957-15962.

Popova EY, Pinzon-Guzman C, Salzberg AC, Zhang SSM, Barnstable CJ. 2016. LSD1-Mediated Demethylation of H3K4me2 Is Required for the Transition from Late Progenitor to Differentiated Mouse Rod Photoreceptor. Molecular neurobiology 53(7): 4563-4581.

Postiglione MP, Juschke C, Xie Y, Haas GA, Charalambous C, Knoblich JA. 2011. Mouse inscuteable induces apical-Basal spindle orientation to facilitate intermediate progenitor generation in the developing neocortex. Neuron 72(2): 269-284.

Puschendorf M, Terranova R, Boutsma E, Mao XH, Isono KI, Brykczynska U, Kolb C, Otte AP, Koseki H, Orkin SH, et al. 2008. PRC1 and Suv39h specify 
parental asymmetry at constitutive heterochromatin in early mouse embryos. Nature Genetics 40(4): 411-420.

Phelan ML, Sif S, Narlikar GJ, Kingston RE. 1999. Reconstitution of a Core Chromatin Remodeling Complex from SWI/SNF Subunits. Molecular cell 3(2): 247-253.

Reik W. 2007. Stability and flexibility of epigenetic gene regulation in mammalian development. Nature 447: 425-432.

Reinke H, Hörz W. 2003. Histones Are First Hyperacetylated and Then Lose Contact with the Activated PHO5 Promoter. Molecular cell 11(6): 1599-1607.

Ronan JL, Wu W, Crabtree GR. 2013. From neural development to cognition: unexpected roles for chromatin. Nature reviews. Genetics 14(5): 347-359.

Ross-Innes CS, Stark R, Teschendorff AE, Holmes KA, Ali HR, Dunning MJ, Brown GD, Gojis O, Ellis IO, Green AR. 2012. Differential oestrogen receptor binding is associated with clinical outcome in breast cancer. Nature 481(7381): 389-393.

Saha A, Wittmeyer J, Cairns BR. 2002. Chromatin remodeling by RSC involves ATPdependent DNA translocation. Genes \& development 16(16): 2120-2134.

Sahara S, O'Leary DD. 2009. Fgf10 regulates transition period of cortical stem cell differentiation to radial glia controlling generation of neurons and basal progenitors. Neuron 63(1): 48-62.

Shen L, Shao N, Liu X, Nestler E. 2014. ngs. plot: Quick mining and visualization of next-generation sequencing data by integrating genomic databases. BMC genomics 15(1): 284.

Shen X, Liu Y, Hsu YJ, Fujiwara Y, Kim J, Mao X, Yuan GC, Orkin SH. 2008. EZH1 mediates methylation on histone $\mathrm{H} 3$ lysine 27 and complements EZH2 in maintaining stem cell identity and executing pluripotency. Molecular cell 32(4): 491-502. 
Shi Y. 2007. Histone lysine demethylases: emerging roles in development, physiology and disease. Nature reviews. Genetics 8(11): 829-833.

Shi Y, Lan F, Matson C, Mulligan P, Whetstine JR, Cole PA, Casero RA. 2004. Histone demethylation mediated by the nuclear amine oxidase homolog LSD1. Cell 119(7): 941-953.

Sokpor G, Castro-Hernandez R, Rosenbusch J, Staiger JF, Tran T. 2018. ATPDependent Chromatin Remodeling During Cortical Neurogenesis. Front Neurosci 12(226).

Sokpor G, Xie Y, Rosenbusch J, Tran T. 2017. Chromatin Remodeling BAF (SWI/SNF) Complexes in Neural Development and Disorders. Frontiers in molecular neuroscience 10: 243-243.

Strahl BD, Allis CD. 2000. The language of covalent histone modifications. Nature 403(6765): 41-45.

Sun G, Alzayady K, Stewart R, Ye P, Yang S, Li W, Shi Y. 2010. Histone demethylase LSD1 regulates neural stem cell proliferation. Mol Cell Biol 30(8): 1997-2005.

Takahashi T, Nowakowski RS, Caviness VS, Jr. 1995. The cell cycle of the pseudostratified ventricular epithelium of the embryonic murine cerebral wall. The Journal of neuroscience : the official journal of the Society for Neuroscience 15(9): 6046-6057.

Takawa M, Masuda K, Kunizaki M, Daigo Y, Takagi K, Iwai Y, Cho HS, Toyokawa G, Yamane Y, Maejima $\mathrm{K}$, et al. 2011. Validation of the histone methyltransferase EZH2 as a therapeutic target for various types of human cancer and as a prognostic marker. Cancer science 102(7): 1298-1305.

Tang L, Nogales E, Ciferri C. 2010. Structure and function of SWI/SNF chromatin remodeling complexes and mechanistic implications for transcription. Progress in biophysics and molecular biology 102(2-3): 122-128. 
Tran T, Dere E, Radyushkin K, Pham L, Nguyen H, Tonchev AB, Sun G, Ronnenberg A, Shi Y, Staiger JF, et al. 2017. Ablation of BAF170 in Developing and Postnatal Dentate Gyrus Affects Neural Stem Cell Proliferation, Differentiation, and Learning. Molecular neurobiology 54(6): 4618-4635.

Tran TC, Boretius S, Sansom SN, Pitulescu ME, Frahm J, Livesey FJ, Stoykova A. 2013. Chromatin regulation by BAF170 controls cerebral cortical size and thickness. Developmental Cell 25(3): 256-269.

Tran TC, Narayanan R, Stoykova A. 2013. BAF chromatin remodeling complex: cortical size regulation and beyond. Cell cycle (Georgetown, Tex.) 12(18): 2953-2959.

Tran TC, Pavlakis E, Tylkowski MA, Stoykova A. 2014. Control of cerebral size and thickness. Cellular and molecular life sciences : CMLS 71(17): 3199-3218.

Tran TC, Radyushkin K, Tonchev AB, Pinon MC, Ashery-Padan R, Molnar Z, Davidoff MS, Stoykova A. 2009. Selective cortical layering abnormalities and behavioral deficits in cortex-specific Pax6 knock-out mice. Journal of Neuroscience 29(26): 8335-8349.

Tran TC, Stoykova A. 2008. Trim11 modulates the function of neurogenic transcription factor Pax6 through ubiquitin-proteosome system. Genes \& development 22(14): 1972-1986.

Varambally S, Cao Q, Mani RS, Shankar S, Wang X, Ateeq B, Laxman B, Cao X, Jing X, Ramnarayanan K, et al. 2008. Genomic loss of microRNA-101 leads to overexpression of histone methyltransferase EZH2 in cancer. Science 322(5908): 1695-1699.

Vasileiou G, Ekici AB, Uebe S, Zweier C, Hoyer J, Engels H, Behrens J, Reis A, Hadjihannas MV. 2015. Chromatin-Remodeling-Factor ARID1B Represses Wnt/ß-Catenin Signaling. American journal of human genetics 97(3): 445-456. 
Veeman MT, Slusarski DC, Kaykas A, Louie SH, Moon RT. 2003. Zebrafish prickle, a modulator of noncanonical $\mathrm{Wnt} / \mathrm{Fz}$ signaling, regulates gastrulation movements. Current biology : $C B$ 13(8): 680-685.

Wang W, Côté J, Xue Y, Zhou S, Khavari PA, Biggar SR, Muchardt C, Kalpana GV, Goff SP, Yaniv M, et al. 1996a. Purification and biochemical heterogeneity of the mammalian SWI-SNF complex. The EMBO journal 15(19): 5370-5382.

Wang W, Xue Y, Zhou S, Kuo A, Cairns BR, Crabtree GR. 1996b. Diversity and specialization of mammalian SWI/SNF complexes. Genes \& development 10(17): 2117-2130.

Watson LA, Tsai L-H. 2017. In the loop: how chromatin topology links genome structure to function in mechanisms underlying learning and memory. Current Opinion in Neurobiology 43: 48-55.

Wen S, Li H, Liu J. 2009. Epigenetic background of neuronal fate determination. Prog Neurobio/ 87(2): 98-117.

Whitehouse I, Flaus A, Cairns BR, White MF, Workman JL, Owen-Hughes T. 1999. Nucleosome mobilization catalysed by the yeast SWI/SNF complex. Nature 400(6746): 784-787.

Woodhead GJ, Mutch CA, Olson EC, Chenn A. 2006. Cell-autonomous beta-catenin signaling regulates cortical precursor proliferation. J Neurosci 26(48): 1262012630.

Wrobel CN, Mutch CA, Swaminathan S, Taketo MM, Chenn A. 2007. Persistent expression of stabilized beta-catenin delays maturation of radial glial cells into intermediate progenitors. Dev Biol 309(2): 285-297.

Wu H, Zhang Y. 2014. Reversing DNA methylation: mechanisms, genomics, and biological functions. Cell 156(1-2): 45-68.

Wu JI. 2012. Diverse functions of ATP-dependent chromatin remodeling complexes in development and cancer. Acta biochimica et biophysica Sinica 44(1): 54-69. 
Wu JI, Lessard J, Olave IA, Qiu Z, Ghosh A, Graef IA, Crabtree GR. 2007. Regulation of Dendritic Development by Neuron-Specific Chromatin Remodeling Complexes. Neuron 56(1): 94-108.

Xiang Y, Zhu Z, Han G, Lin H, Xu L, Chen CD. 2007. JMJD3 is a histone H3K27 demethylase. Cell Research 17(10): 850-857.

Yan Z, Wang Z, Sharova L, Sharov AA, Ling C, Piao Y, Aiba K, Matoba R, Wang W, Ko MS. 2008. BAF250B-associated SWI/SNF chromatin-remodeling complex is required to maintain undifferentiated mouse embryonic stem cells. Stem Cells 26(5): 1155-1165.

Yao B, Christian KM, He C, Jin P, Ming G-I, Song H. 2016. Epigenetic mechanisms in neurogenesis. Nature Reviews Neuroscience 17: 537-549.

Yao B, Christian KM, He C, Jin P, Ming GL, Song H. 2016. Epigenetic mechanisms in neurogenesis. Nature reviews. Neuroscience 17(9): 537-549.

Yoo AS, Crabtree GR. 2009. ATP-dependent chromatin remodeling in neural development. Current Opinion in Neurobiology 19(2): 120-126.

Zhang J, Parvin J, Huang K. 2012. Redistribution of H3K4me2 on neural tissue specific genes during mouse brain development. BMC Genomics 13 Suppl 8: S5.

Zhou CJ, Borello U, Rubenstein JL, Pleasure SJ. 2006. Neuronal production and precursor proliferation defects in the neocortex of mice with loss of function in the canonical Wnt signaling pathway. Neuroscience 142(4): 1119-1131.

Zhuo L, Theis M, Alvarez-Maya I, Brenner M, Willecke K, Messing A. 2001. hGFAPcre transgenic mice for manipulation of glial and neuronal function in vivo. Genesis 31(2): 85-94. 


\section{List of figures}

Figure 1.1 Chromatin remodeling BAF (mSWI/SNF) complex in neural development.....1

Figure 1.2. Model show the deletion of BAF complex lead to dissociation 5

Figure 2.1. The expressions of BAF155 and BAF170 are indispensable for embryonic development. 12

Figure 2.2. Expression of BAF subunits in telencephalon-specific dcKO_FoxG1-Cre mutants

Figure 2.3. Expression of BAF subunits in embryos of TAM-inducible full dcKO_CAGCre mutants 15

Figure 2.4. BAF complexes control the level of H3K27me3 in the brain and whole embryo during development

Figure 3.1. BAF complexes globally control epigenetic and gene expression programs in late development pallium .26

Figure 3.2. H3K27me3-linked silencing of neuronal differentiation-related genes in BAF complex-deleted pallium in late stages .29

Figure 3.3. BAF complexes are required for the formation of cortical upper layers and the hippocampus 32

Figure 3.4. Loss of BAF155 and BAF170 causes H3K4me2-linked upregulation of genes involved in the mitotic cell cycle and proliferation in late cortical development .35

Figure 3.5. NE-like cells in the BAF-complex-deleted pallium in late development retain their highly proliferative competence 36 
Figure 3.6. BAF complexes suppress Wnt signaling activity .43

Figure 3.7. BAF complexes control hippocampal development by suppressing Wnt signaling activity .46

Figure S3.1. Co-expression of BAF155/BAF170 with H3K4 and H3K27 demethylases in the developing pallium .58

Figure S3.2. Characterization of H3K27me3 and H3K4me2 marks in dcKO_hGFAP-Cre cortex 60

Figure S3.3. BAF155 and BAF170 are essential for neurogenesis in the developing cortex and hippocampus .62

Figure S3.4. Elevated level of H3K27me3 and H3K4me2 by inhibition of H3K27 and H3K4me2 demethylases caused the defect in neuronal differentiation in developing pallium 64

Figure S3.5. Loss of BAF155 and BAF170 in dcKO mutants has a profound effect on the pool of NSCs, expression of adherens junction molecules .66

Figure S3.6. The spindle orientation, selective apoptosis of RGs in dcKO_hGFAP-Cre mutants and phenotypes of cortical neuron-specific dcKO_Nex-Cre mutants .68

Figure S3.7. Suppression of Wnt signaling in the developing pallium by BAF complexes in the developing forebrain .70 


\section{Abbreviations}

\begin{tabular}{|c|c|}
\hline ATP & Adenosine triphosphate \\
\hline BAF & $\underline{B}$ rg1/Brm associated factor \\
\hline BLBP & Brain lipid binding protein \\
\hline cDNA & Complementary deoxyribonucleic acid \\
\hline ChIP-seq & $\underline{\text { Chromatin immunoprecipitation sequencing }}$ \\
\hline $\mathrm{cKO}$ & Conditional knockout \\
\hline Cx & $\underline{\text { Cortex }}$ \\
\hline CA & cornu ammonis \\
\hline $\mathrm{CP}$ & Cortical plate \\
\hline $\mathrm{CHD}$ & 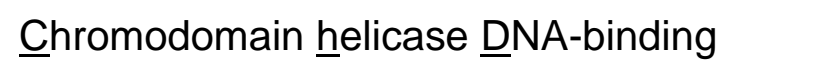 \\
\hline ColP & co-immunoprecipitation \\
\hline DAPI & 4,6-dasmindino-2-phenylindol \\
\hline DG & Dentate gyrus \\
\hline dcKO & Double conditional knockout \\
\hline DMEM & Dulbecco's Modified Eagle Medium \\
\hline DNA & Deoxyribonucleic acid \\
\hline $\mathrm{DP}$ & Dorsal pallium \\
\hline eGFP & Enhanced green fluorescent protein \\
\hline Emx1 & Empty spiracles homeobox 1 \\
\hline $\mathrm{E}$ & Embryonic day \\
\hline ESCs & Émbryonic stem cells \\
\hline $\mathrm{Fl}$ & $\underline{\text { Floxed }}$ \\
\hline FOP & Super8XFOPFLASH \\
\hline GFP & Green fluorescent protein \\
\hline GLAST & GLutamate Aspartate Transporter \\
\hline
\end{tabular}




\begin{tabular}{|c|c|}
\hline GO & Gene ontology \\
\hline GTPases & Guanosine tri-phosphatases \\
\hline h & hours \\
\hline Hprt1 & hypoxanthine phosphoribosyltransferase \\
\hline $\mathrm{Hi}$ & Hippocampus \\
\hline hGFAP & Human glial fibrillary acidic protein \\
\hline $\mathrm{IHC}$ & Immune histochemistry \\
\hline IncRNA & Long non-coding RNA \\
\hline IF & Intensity fluorescence \\
\hline $\lg G$ & Immunoglobulin $\underline{\mathrm{G}}$ \\
\hline IPs & Intermediate progenitor cells \\
\hline IUE & $\underline{\text { In }}$ utero electroporation \\
\hline IZ & Intermediate żone \\
\hline L & Layer \\
\hline LP & Lateral pallium \\
\hline LL & Lower layer \\
\hline NSC & Neural stem cell \\
\hline NE & Neuroepithelial \\
\hline NuRD & Nucleosome $\underline{\text { Remodeling Deacetylase }}$ \\
\hline MP & Medial pallium \\
\hline$P$ & Postnatal stage \\
\hline Pax6 & 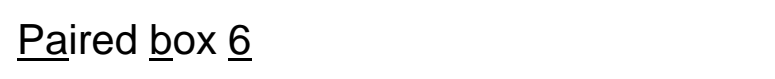 \\
\hline PBS & Phosphate Buffer $\underline{\text { Saline }}$ \\
\hline PCR & Polymerase chain reaction \\
\hline $\mathrm{pHH} 3$ & Phosphorylated histone $\underline{3}$ \\
\hline PVim & Phosphorylated vimentin \\
\hline qPCR & Quantitative Polymerase chain reaction \\
\hline RGs & Radial glial cells \\
\hline
\end{tabular}




\begin{tabular}{|c|c|}
\hline rpm & $\underline{\text { Revolutions per minute }}$ \\
\hline RNA-seq & $\underline{\text { Ribonucleic acid sequencing }}$ \\
\hline SEM & $\underline{\text { Standard }}$ error of the mean \\
\hline SDS & Sodium dodecyl sulphate \\
\hline sh & Short hairpin \\
\hline Sox2 & Transcription factor $\underline{\text { SOX2 }}$ \\
\hline SVZ & Sub-ventricular zone \\
\hline TAM & $\underline{\text { Tamoxifen }}$ \\
\hline Tbr1 & I-box $\underline{\text { brain } \underline{1}}$ \\
\hline Tbr2 & T-box brain $\underline{2}$ \\
\hline TF & Iranscription factor \\
\hline TOP & 8XTOPFLASH \\
\hline TSS & Transcription start site \\
\hline TES & Transcription end site \\
\hline UL & Unpper layer \\
\hline Veh & Vehicle \\
\hline VZ & Ventricular zone \\
\hline WB & $\underline{\text { Western blot }}$ \\
\hline WT & Wildtype \\
\hline WIG & $\underline{\text { Wiggle }}$ \\
\hline 2-PCPA & Trans-2- $\underline{P}$ henylcyclopropylamine hydrochloride \\
\hline $3 D$ & 3-dimensional \\
\hline
\end{tabular}


Abbreviations 


\section{Curriculum vitae}

\section{Personal details:}

Name: $\quad$ Thi-Huong Nguyen

Date of birth: $\quad$ April $10^{\text {th }}, 1984$

Place of birth: $\quad$ Bac Giang

Nationality: $\quad$ Vietnam

\section{Education:}

02.2015-Present: PhD student in the lab of Prof. Dr. Jochen Staiger at Department of Neuroanatomy, University Medical Center Goettingen.

09.2009-12.2011: Master of Science in Experimental Biotechnology at Thai Nguyen University, Thai Nguyen, Vietnam

09.2003-07.2007: Bachelor of Science at Thai Nguyen University of Agriculture, Thai Nguyen, Vietnam

\section{Publications:}

Nguyen $\mathbf{H}^{\star}$, Kerimoglu $\mathrm{C}^{*}$, Pirouz M, Pham L, Kiszka KA, Sokpor G, Sakib MS, Rosenbusch J, Teichmann U, Seong RH, Stoykova A, Fischer A, Staiger JF, Tran T. 2018. Epigenetic Regulation by BAF Complexes Limits Neural Stem Cell Proliferation by Suppressing Wnt Signaling in Late Embryonic Development. Stem cell reports 10(6): 1734-1750. 
Nguyen $\mathbf{H}^{*}$, Sokpor G*, Pham L, Rosenbusch J, Stoykova A, Staiger JF, Tran T. 2016. Epigenetic regulation by BAF ( $\mathrm{mSWl} / \mathrm{SNF}$ ) chromatin remodeling complexes is indispensable for embryonic development. Cell cycle 15(10): 1317-1324.

Bachmann $\mathrm{C}^{*}$, Nguyen $\mathbf{H}^{*}$, Rosenbusch $\mathrm{J}^{*}$, Pham L, Rabe T, Patwa M, Sokpor G, Seong RH, Ashery-Padan R, Mansouri A, Stoykova A,Staiger JF, Tran T. 2016. $\mathrm{mSWI} / \mathrm{SNF}$ (BAF) Complexes Are Indispensable for the Neurogenesis and Development of Embryonic Olfactory Epithelium. PLOS Genetics 12(9): e1006274.

Tran T, Dere E, Radyushkin K, Pham L, Nguyen H, Tonchev AB, Sun G, Ronnenberg A, Shi Y, Staiger JF, Ehrenreich H, Stoykova A. 2017. Ablation of BAF170 in Developing and Postnatal Dentate Gyrus Affects Neural Stem Cell Proliferation, Differentiation, and Learning. Molecular neurobiology 54(6): 4618-4635.

*Equally contributing first author 